TOPICAL REVIEW

\title{
Intermediate and extreme mass-ratio inspirals—astrophysics, science applications and detection using LISA
}

\author{
Pau Amaro-Seoane ${ }^{1}$, Jonathan R Gair ${ }^{2}$, Marc Freitag'2, \\ M Coleman Miller ${ }^{3}$, Ilya Mandel ${ }^{4}$, Curt J Cutler ${ }^{5}$ and Stanislav Babak ${ }^{1}$ \\ ${ }^{1}$ Max-Planck-Institut für Gravitationsphysik (Albert Einstein-Institut), Am Mühlenberg 1, \\ D-14476 Potsdam, Germany \\ 2 Institute of Astronomy, Madingley Road, Cambridge CB3 OHA, UK \\ 3 Department of Astronomy, University of Maryland, College Park, MD 20742-2421, USA \\ 4 Theoretical Astrophysics, California Institute of Technology, Pasadena, CA 91125, USA \\ 5 Jet Propulsion Laboratory, California Institute of Technology, Pasadena, CA 91109, USA \\ E-mail: pau@aei.mpg.de,jrg23@cam.ac.uk,freitag@cam.ac.uk,miller@astro.umd.edu, \\ ilya@caltech.edu, curt.j.cutler@jpl.nasa.gov and stba@aei.mpg.de
}

Received 19 March 2007, in final form 26 June 2007

Published 9 August 2007

Online at stacks.iop.org/CQG/24/R113

\begin{abstract}
Black hole binaries with extreme $\left(\gtrsim 10^{4}: 1\right)$ or intermediate $\left(\sim 10^{2}-10^{4}: 1\right)$ mass ratios are among the most interesting gravitational wave sources that are expected to be detected by the proposed laser interferometer space antenna (LISA). These sources have the potential to tell us much about astrophysics, but are also of unique importance for testing aspects of the general theory of relativity in the strong field regime. Here we discuss these sources from the perspectives of astrophysics, data analysis and applications to testing general relativity, providing both a description of the current state of knowledge and an outline of some of the outstanding questions that still need to be addressed. This review grew out of discussions at a workshop in September 2006 hosted by the Albert Einstein Institute in Golm, Germany.
\end{abstract}

PACS numbers: 95.30.Sf, 98.62.Js, 98.10.+z, 04.30.Db, 04.30.-w, 04.25.-g, 04.25.Nx

(Some figures in this article are in colour only in the electronic version)

\section{Contents}

$\begin{array}{ll}\text { 1. Background } & \mathrm{R} 114\end{array}$

$\begin{array}{ll}\text { 2. EMRI astrophysics } & \text { R116 }\end{array}$

$\begin{array}{ll}\text { 2.1. Inspirals of stellar objects into MBHs } & \mathrm{R} 117\end{array}$

$\begin{array}{ll}\text { 2.2. Intermediate-mass black holes and inspirals into MBHs } & \mathrm{R} 125\end{array}$ 


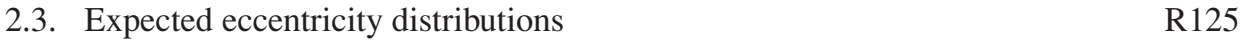

$\begin{array}{ll}\text { 2.4. Numerical stellar dynamics for I/EMRIs } & \text { R128 }\end{array}$

$\begin{array}{ll}\text { 3. EMRI detection } & \text { R139 }\end{array}$

$\begin{array}{ll}\text { 3.1. Data analysis algorithms } & \text { R139 }\end{array}$

$\begin{array}{ll}\text { 3.2. Source modelling } & \text { R143 }\end{array}$

$\begin{array}{lr}\text { 4. Testing relativity theory } & \text { R149 }\end{array}$

$\begin{array}{ll}\text { 4.1. Current status } & \text { R150 }\end{array}$

$\begin{array}{ll}\text { 4.2. Outstanding challenges } & \text { R153 }\end{array}$

5. EMRI science $\quad$ R155

5.1. What can we learn from the characterizations of EMRI/IMRI dynamics, i.e., the observed eccentricities etc of the orbits?

5.2. What can we learn about the inspiralling compact objects from EMRIs/ IMRIs?

5.3. What can we learn about the MBHs from EMRIs/IMRIs?

5.4. What can we learn about cosmology and early structure formation from EMRIs/IMRIs?

5.5. How can EMRIs/IMRIs be used to test GR, or (assuming GR is correct) that the central massive object is a Kerr BH?

$\begin{array}{ll}\text { References } & \text { R163 }\end{array}$

\section{Background}

Our understanding of the central regions of galaxies has advanced rapidly during the past few years, not least due to major advances in high angular resolution instrumentation at a variety of wavelengths. Observations carried out with space-borne telescopes, such as the Hubble Space Telescope (HST), and from the ground, using adaptive optics, have allowed the study of the kinematics of stars or gas in regions reaching down to milli-pc for the Milky Way and to sub-pc scales for more distant galaxies. One remarkable conclusion is that dark compact objects, most probably massive black holes (MBHs), with a mass $\mathcal{M}_{\bullet} \simeq 10^{6}-10^{9} M_{\odot}$, are present at the centre of most of the galaxies for which such observations can be made. A deep link exists between the central MBH and the host galaxy. This is exemplified by the discovery of correlations between $\mathcal{M}_{\bullet}$ and global properties of the spheroid, the tightest correlation being with its velocity dispersion, the so-called $\mathcal{M}_{\bullet}-\sigma$ relation $[91,308]$. The central part of a galaxy, its nucleus, consists of a cluster of a few $10^{7}$ to a few $10^{8}$ stars surrounding the $\mathrm{MBH}$, with a size of a few pc. The nucleus is understandably expected to play a major role in the interaction between the $\mathrm{MBH}$ and the host galaxy. In the nucleus, one finds stellar densities in excess of $10^{6} \mathrm{pc}^{-3}$ and relative velocities of order a few $100 \mathrm{~km} \mathrm{~s}^{-1}$ to a few $1000 \mathrm{~km} \mathrm{~s}^{-1}$. In these exceptional conditions and unlike anywhere else in the bulk of the galaxy, collisional effects come into play. These include two-body relaxation, i.e., mutual gravitational deflections and genuine contact collisions.

The stars and the MBH interact in two primary ways. Firstly, stars can produce gas to be accreted onto the $\mathrm{MBH}$, through normal stellar evolution, collisions or disruptions of stars by the strong central tidal field. These processes may contribute significantly to the mass of the $\mathrm{MBH}[106,241]$. Tidal disruptions trigger phases of bright accretion that may reveal the presence of an MBH in an otherwise quiescent, possibly very distant, galaxy [121, 154]. Secondly, stars can be swallowed whole if they are kicked directly through the horizon (referred to as direct plunges) or inspiral gradually due to the emission of gravitational waves (GWs). 
The latter process, known as an 'extreme mass ratio inspiral' (EMRI) is one of the main sources expected for the future space-borne GW detector laser interferometer space antenna (LISA) [72, 306]. For the last stages of an EMRI to produce GWs in the frequency domain to which LISA will be most sensitive, i.e., $0.1 \mathrm{mHz}-100 \mathrm{mHz}$ [187], the mass of the MBH must be between $\sim 10^{4} M_{\odot}$ and $\sim 10^{7} M_{\odot}$. Only compact stars, i.e., white dwarfs, neutron stars, stellar mass black holes or, possibly, the Helium cores of giant stars can produce EMRI signals detectable at extra-galactic distances. Main-sequence stars are either not compact enough to withstand the tidal forces in the vicinity of the MBH or not massive enough to produce waves of large enough amplitude. Predictions for the expected number of EMRI detections that LISA will make are rather uncertain but lie in the range of a few to a few thousand.

On the other hand, numerical simulations of young dense clusters show that runaway collisions due to mass segregation can produce central stars with masses $\sim 10^{2-4} M_{\odot}$ $[109,139,260,263]$. Such a star might undergo collapse and form a so-called intermediatemass black hole (IMBH) with $M \sim 10^{2-4} M_{\odot}$. It has also been proposed that globular clusters can capture the compact remnant of a zero-metallicity population III star [12]. A cluster harbouring an IMBH which starts relatively close to the central $\mathrm{MBH}$ of the host galaxy will sink to the centre in a few million years, and will eventually release its central IMBH due to tidal stripping of the cluster [83]. A first-order estimate of the event rate of this process leads to a few detectable coalescences of IMBHs with MBHs per year in the universe [227] but even if only one of these events occurs during the LISA mission, the signal-to-noise ratio by the end of the inspiral would be so high $[71,131,165]$ that it would be visible in a time-frequency spectrogram of the LISA data, without having to resort to matched filtering [227]. The mass ratio of such a merger would typically be $10^{3-4}: 1-$ we shall refer to it as an intermediate mass ratio inspiral' (IMRI) for obvious reasons.

The LISA mission is scheduled to fly in about ten years and critical design choices which will affect the ability of detecting E/IMRIs will be made soon. It is important to produce robust estimates for the rates and typical orbital parameters of these events as input for the development of search algorithms. Such search algorithms must have the capability of extracting science information out of the complex LISA data stream, which will contain many thousands of overlapping resolvable signals, plus astrophysical backgrounds from millions of more distant sources. The readiness of data analysis for the LISA mission will be assessed on an equal footing with the hardware, so it is essential that data analysis strategies are finalized in the near future.

Detection of EMRIs with LISA is difficult (in discussions of data analysis, we will generally use the term EMRIs to refer to both EMRIs and IMRIs since most of the discussion applies to both types of inspirals, except for those obvious cases when we discuss differences between the two). A typical signal will be very weak, lying buried in instrumental noise and in the gravitational wave foreground created by nearby Galactic white dwarf binaries. The signals are long-lived, typically being observable for several years prior to plunge, which in principle allows the EMRIs to be detected by matched filtering. Matched filtering employs a bank of templates that describes signals with all possible parameters within the expected range. Unfortunately, the large parameter space of possible EMRIs makes the number of templates required for such a search computationally prohibitive. Over the past few years, several alternative algorithms have been developed-a semi-coherent matched filtering algorithm, time-frequency search algorithms and Markov Chain Monte Carlo techniques. The results are promising, but more work needs to be done before we will have an optimal algorithm for EMRI detection. The correspondingly higher signal-to-noise ratios of IMRIs make detection of those events somewhat easier, but it is still a challenging task. 
The structure of this paper is as follows. In section 2, we present the various astrophysical circumstances that might lead to the formation of EMRI sources. The simplest and most studied situation is that of a dense, spherical galactic nucleus where two-body relaxation brings compact stars on to very eccentric orbits around an MBH. Gravitational wave emission may shrink the orbit and create an EMRI but relaxation can hinder this evolution by increasing the pericentre distance. We present this standard picture and several processes that complicate it and make the EMRI rate rather uncertain. We then mention several other EMRI- and IMRIformation channels which have been recently proposed but remain to be investigated in more detail.

Predictions for the rates and characteristics of EMRIs rely heavily on numerical modelling of dynamics of dense stellar systems. In section 2.4, we explain the methods that are in use or have been used in stellar dynamical studies applied to EMRIs. We outline the limitations of these approaches and suggest avenues for future numerical work in that field.

In section 3.1 we describe the existing algorithms and outstanding challenges for EMRI/IMRI detection. The search algorithms require models of EMRI waveforms. In principle, the extreme mass ratio means that the waveforms can be computed using black hole perturbation theory. However, this formalism is not fully developed and will be computationally expensive once it is. Various alternative waveform models are currently being developed and used for scoping out EMRI detection. We will describe these various models and necessary future developments of them in section 3.2.

If we do detect many EMRI/IMRI events, we will be able to do some very interesting science. EMRI observations will provide measurements of the masses and spins of black holes to an accuracy which is not accessible by other astronomical observations. This will tell us about the properties and growth of black holes in the nearby universe. EMRIs also provide a means to probe general relativity in the strong-field regime close to astrophysical black holes. The extreme mass ratio ensures that over many orbits the inspiralling object acts essentially like a test body moving in the spacetime of the central body. The emitted gravitational waves encode a map of the spacetime. If we can decode that map, then we will be able to test the belief that massive compact objects in the centres of galaxies are indeed Kerr black holes. Carrying out this mapping is difficult, but is the focus of much current research. We summarize current results in section 4 and discuss some outstanding questions in this area.

In section 5 we discuss the scientific benefits of LISA observations of EMRI/IMRI events. This section takes the form of answers to five broad questions that were the focus of discussions at the LISA EMRI workshop hosted by the Albert Einstein Institute in Golm, Germany in September, 2006. Finally, in section 6 we provide a summary of the main topics in the paper.

\section{EMRI astrophysics}

Astrophysical scenarios producing EMRI events and their relative rates are currently rather uncertain, but multiple physical channels are being explored. We will begin by discussing the 'standard' picture of these events, in which a single $10 M_{\odot}$ black hole spirals into a supermassive black hole via gravitational wave emission, and the only mechanism by which the angular momentum is changed is through two-body relaxation. Even within this restricted picture there are substantial unknowns about the rates. We follow this by discussing possible modifications of this basic picture, including the effects of resonant relaxation, triaxiality and mass segregation. We then consider different 'non-standard' processes that can lead to EMRIs, including tidal separation of black hole binaries and formation or capture of massive stars in accretion discs.

Our focus will then switch to IMRIs. If even a single such event is observed with LISA, the strength of the signal could be enough to yield unique information about strong gravity, in 
a relatively model-independent way [227]. However, given that the existence of IMBHs has not yet been established with certainty, the unknowns for this scenario are even more daunting than they are for EMRIs. In the second part of this section we discuss the current state of knowledge about IMBHs and IMRIs, and outline problems that need to be addressed in the future.

The third portion of this section deals with numerical methods that are being used to simulate the interactions of black holes in dense nuclear regions. So far only approximate methods using many simplifying assumptions have been used to estimate the rates and characteristics of inspirals of stellar-mass objects into an MBH. We review these approaches, their accomplishments and limitations. Thanks to the rapid increase in computer power and the development of new algorithms, it is likely that the direct $N$-body approach will soon be able to confirm or disprove these approximate results and extend them. However, exceptionally long and accurate integrations are needed to account correctly for secular effects such as resonant relaxation or Kozai oscillations. These requirements pose new challenges to developers of $\mathrm{N}$-body codes.

\subsection{Inspirals of stellar objects into $\mathrm{MBHs}$}

2.1.1. The standard picture. The first detailed calculations of EMRI rates were performed by Hils and Bender [158] and Sigurdsson and Rees [287].

Their simple picture involved the following assumptions.

(1) The stellar-mass compact objects are treated as single objects.

(2) The distribution of stars is spherically symmetric.

(3) The only mechanism that can change the angular momentum of the compact objects is two-body relaxation.

(4) There is no mass segregation, hence the compact objects are distributed spatially the same way as the stars.

(5) There is no gas in the nucleus.

With these assumptions, inside the 'radius of influence'

$$
r_{\text {infl }}=\frac{G \mathcal{M}_{\bullet}}{\sigma_{0}^{2}} \approx 1 \mathrm{pc}\left(\frac{\mathcal{M}_{\bullet}}{10^{6} M_{\odot}}\right)\left(\frac{60 \mathrm{~km} \mathrm{~s}^{-1}}{\sigma_{0}}\right)^{2},
$$

within which the central MBH dominates the gravitational field, the relaxation time is

$t_{\mathrm{rlx}}(r)=\frac{0.339}{\ln \Lambda} \frac{\sigma^{3}(r)}{G^{2}\langle m\rangle m_{\mathrm{CO}} n(r)} \simeq 1.8 \times 10^{8} \mathrm{yr}\left(\frac{\sigma}{100 \mathrm{~km} \mathrm{~s}^{-1}}\right)^{3}\left(\frac{10 M_{\odot}}{m_{\mathrm{CO}}}\right)\left(\frac{10^{6} M_{\odot} \mathrm{pc}^{-3}}{\langle m\rangle n}\right)$.

Here $\sigma(r)$ is the local velocity dispersion. It is approximately equal to the Keplerian orbital speed $\sqrt{G \mathcal{M}_{\bullet} r^{-1}}$ for $r<r_{\text {infl }}$ and has a value $\approx \sigma_{0}$ outside of it. $n(r)$ is the local number density of stars, $\langle m\rangle$ is the average stellar mass, $m_{\mathrm{CO}}$ is the mass of the compact object (we take a standard $m_{\mathrm{CO}}=10 M_{\odot}$ for stellar-mass black holes), and

$$
\ln \Lambda \equiv \ln \left(p_{\max } / p_{0}\right)
$$

is the Coulomb logarithm. Here $p_{\max }$ is an upper limit for the impact parameter $p$; $p_{0}=G\langle m\rangle \sigma^{-2}$ is the value that corresponds to a deflection angle of $\pi / 2$ for stars of masses $\langle m\rangle$ and relative velocity $\sigma[43,289]$. For a self-gravitating stellar system, $\Lambda \equiv p_{\max } / p_{0} \simeq \gamma \mathcal{N}_{\star}$ where $\mathcal{N}_{\star}$ is the number of stars and $\gamma$ is in the range $0.01-0.1$ depending on the mass spectrum $[125,152]$. In the vicinity of the BH $\left(r<r_{\text {infl }}\right), \Lambda \approx \mathcal{M}_{\bullet} / m_{\star}[19,195]$. In any case, 
$\ln \Lambda \sim 10$. For typical density profiles, $t_{\text {rlx }}$ decreases slowly with decreasing $r$ inside $r_{\text {infl }}$. It should be noted that the exchange of energy between stars of different masses-sometimes referred to as dynamical friction in the case of one or a few massive bodies in a field of much lighter objects-occurs on a timescale shorter than $t_{\mathrm{rlx}}$ by a factor of roughly $M /\langle m\rangle$, where $M$ is the mass of a heavy body (e.g. [108, 139] and references therein).

Relaxation redistributes orbital energy amongst stellar-mass objects until the most massive of them (presumably stellar-mass black holes) form a power-law density cusp, $n(r) \propto r^{-\alpha}$ with $\alpha \simeq 1.75$ around the $\mathrm{MBH}$, while less massive species arrange themselves into a shallower profile, with $\alpha \simeq 1.4-1.5$ [10, 19, 36, 82, 106, 108, 163, 195, 216, 267] (see also section 2.4.5). Nuclei likely to host MBHs in the LISA mass range $\left(\mathcal{M}_{\bullet} \lesssim\right.$ few $\left.\times 10^{6} M_{\odot}\right)$ probably have relaxation times comparable to or less than a Hubble time, so that it is expected that their heavier stars form a steep cusp, although nothing is known about the presence of Bahcall-Wolf cusps in galaxies aside from the Milky Way (see discussion in section 2.1.3).

In a spherical potential, at any given time the stars and compact objects in the nucleus simply orbit the MBH with their semi-major axes and eccentricities changing slowly, owing to two-body relaxation. For an EMRI to occur, in this standard picture, two-body relaxation has to bring a compact remnant onto an orbit with such a small pericentre distance that dissipation of energy by emission of GWs becomes significant.

If the object is on a very eccentric orbit but one for which the timescale for passage through periapse, $t_{\text {peri }} \simeq(1-e)^{3 / 2} P$, is less than $\sim 10^{4} \mathrm{~s}$, the source will generate bursts of gravitational radiation in the LISA band each time the object passes through periapse. However, such GW signals consist of bursts which can probably not benefit from coherent signal processing even if they repeat with a periodicity shorter than LISA mission duration. Only if they reside at the Milky Way centre is there a non-vanishing probability for LISA to detect such sources [164]. An extra-galactic source is only likely to be detectable if it radiates continuously in the LISA band. As a rough guide, therefore, a detectable EMRI source must have an orbital frequency higher than about $f_{\text {LISA }}=10^{-4} \mathrm{~Hz}$, corresponding to the condition on the semi-major axis $a \lesssim 0.5 \mathrm{AU}\left[f_{\mathrm{LISA}} /\left(10^{-4} \mathrm{~Hz}\right)\right]^{-2 / 3}\left[\mathcal{M}_{\bullet} /\left(10^{6} M_{\odot}\right)\right]^{1 / 3}$. As there is no sharp cut-off in the predicted LISA sensitivity curve at $10^{-4} \mathrm{~Hz}$, a strong source might be detectable at a lower frequency.

Not all objects with an inspiral time by GW emission shorter than a Hubble time will end up as EMRIs. This is because, although relaxation can increase the eccentricity of an object to very high values, it can also perturb the orbit back to a more circular one for which GW emission is completely negligible. Typically, neglecting GW emission, it takes a time of order $t_{\mathrm{rlx}} \ln (1-e)$ for an orbit to reach a (large) eccentricity $e$ through the effects of two-body relaxation. However, the pericentre distance $R_{\mathrm{p}}=a(1-e)$ can be significantly altered by relaxation on a timescale $t_{\mathrm{rel}, \mathrm{p}} \simeq(1-e) t_{\mathrm{rlx}}$, so the condition for a star to become an EMRI is that it moves onto an orbit for which the timescale for orbital decay by GW emission, $\tau_{\mathrm{GW}}$ (see equation (11)) is sufficiently shorter than $(1-e) t_{\mathrm{rlx}}$. If the semi-major axis of the orbit is too large, this condition cannot be obeyed unless the star actually finds itself on an unstable, plunging orbit, with $e \geqslant e_{\mathrm{pl}}(a) \equiv 1-4 R_{\mathrm{S}} / a$, where $R_{\mathrm{S}}$ is the Schwarzschild radius of the MBH. The very short burst of gravitational radiation emitted during a plunge through the horizon can only be detected if originating from the Galactic centre [164]. Coherent integration of the GW signal for $>10^{4}$ cycles with a frequency in LISA band is required for detection of extragalactic EMRIs.

The situation for EMRI production in the standard picture is more complicated than that of tidal disruptions by the MBH (e.g., [201, 273, 298, 317]) or GW bursts from stars on very eccentric orbits $[164,276]$ because these processes require a single passage within a well-defined distance $R_{\text {enc }}$ from the $\mathrm{MBH}$ to be 'successful'. In such cases, at any distance 
from the centre and for any given modulus of the velocity, there exists a 'loss cone' inside which the velocity vector of a star has to point for it to pass within $R_{\text {enc }}$ of the MBH $[8,20,97,195]$. In contrast, an EMRI is a progressive process which will only be successful (as a potential source for LISA) if the stellar object experiences a very large number of successive dissipative close encounters with the MBHs [6]. There is no well-defined loss cone for such a situation.

As described above, a source becomes an EMRI when the orbital period becomes shorter than about $10^{4} \mathrm{~s}$. It seems unlikely that the evolution of such a tight orbit can be significantly affected by other stars or the ambient gas. It is not so at earlier stages of the inspiral as twobody relaxation, experienced mostly at apocentre can easily induce a change in the pericentre distance large enough to either render GW emission completely insignificant or, in contrast, cause a sudden plunge into the MBH $[158,161]$. The condition for successful inspiral is not that the pericentre distance must be sufficiently small, like for tidal disruptions or GW bursts, but that the timescale for orbit evolution by emission of GWs (see equation (11)) is sufficiently shorter than the timescale over which two-body relaxation can affect the pericentre distance significantly

$$
\tau_{\mathrm{GW}}<C_{\mathrm{EMRI}}(1-e) t_{\mathrm{rlx}} .
$$

What 'sufficiently shorter' means is the crux of the problem and is encoded in $C_{\text {EMRI }}$, a 'safety' numerical constant that makes this condition sufficient $\left(C_{\text {EMRI }}<1\right)$. For a given semi-major axis $a$, one can define a critical eccentricity $\tilde{e}(a)$ above which GW emission dominates over orbital evolution due to relaxation and a corresponding time scale $\tilde{\tau}(a) \equiv \tau_{\mathrm{GW}}(\tilde{e}, a) \equiv C_{\mathrm{EMRI}}(1-\tilde{e}) t_{\mathrm{rlx}}$. Plunging orbits have $e \geqslant e_{\mathrm{pl}}(a) \equiv 1-4 R_{\mathrm{S}} / a$ so EMRIs (as opposed to direct plunges) can only happen if $e_{\mathrm{pl}}(a)>\tilde{e}(a)$. This defines a critical semi-major axis which is a typical value for an EMRI at the moment orbital evolution starts being dominated by GW emission

$a_{\mathrm{EMRI}}=5.3 \times 10^{-2} \mathrm{pc} C_{\mathrm{EMRI}}^{2 / 3} \times\left(\frac{t_{\mathrm{rlx}}}{10^{9} \mathrm{yr}}\right)^{2 / 3}\left(\frac{m}{10 M_{\odot}}\right)^{2 / 3}\left(\frac{\mathcal{M}_{\bullet}}{10^{6} M_{\odot}}\right)^{-1 / 3}$.

The corresponding eccentricity is given by

$1-e_{\mathrm{EMRI}}=7.2 \times 10^{-6} C_{\mathrm{EMRI}}^{-2 / 3} \times\left(\frac{t_{\mathrm{rlx}}}{10^{9} \mathrm{yr}}\right)^{-2 / 3}\left(\frac{m}{10 M_{\odot}}\right)^{-2 / 3}\left(\frac{\mathcal{M}_{\bullet}}{10^{6} M_{\odot}}\right)^{4 / 3}$.

The situation is represented in figure 1 in the semi-major axis, eccentricity plane. We plot schematically the trajectory for a typical EMRI evolving according to the standard scenario (labelled 'one-body inspiral' to distinguish it from the binary tidal separation scenario discussed later). Initially the values of $a$ and $e$ random walk due to two-body relaxation. As it takes of order $t_{\mathrm{rlx}}$ to change $a$ by a factor of 2 but only $(1-e) t_{\mathrm{rlx}}$ to change the value of $1-e$ (and hence the periapse), the random walk seems more and more elongated in the horizontal direction, the smaller the value of $1-e$. It is much more likely for a star to cross over to the plunging or GW-dominated region by acquiring a very high eccentricity than by shrinking $a$ significantly. Typically an EMRI 'progenitor' starts with a semi-major axis slightly lower than $a_{\mathrm{EMRI}}$. It takes on average a time of order $\ln (1-\tilde{e})^{-1} t_{\mathrm{rlx}} \simeq 10 t_{\mathrm{rlx}}$ for relaxation to produce an eccentricity such that GW emission becomes dominant. From that point, the object will follow a path closer and closer to a pure inspiral (approximated by Peters equations [253], see section 2.3.1).

At larger $a$ values, inspirals are practically impossible because GW emission is not significant in comparison to relaxation even on plunge orbits. Unless they first shrink their orbit through two-body relaxation, these objects will be swallowed by the $\mathrm{MBH}$ on a direct plunge. Inspirals starting with $a \ll a_{\text {EMRI }}$ are rare because, for a density cusp $n \propto r^{-\alpha}$ with $\alpha \simeq 1.4-1.8$ 


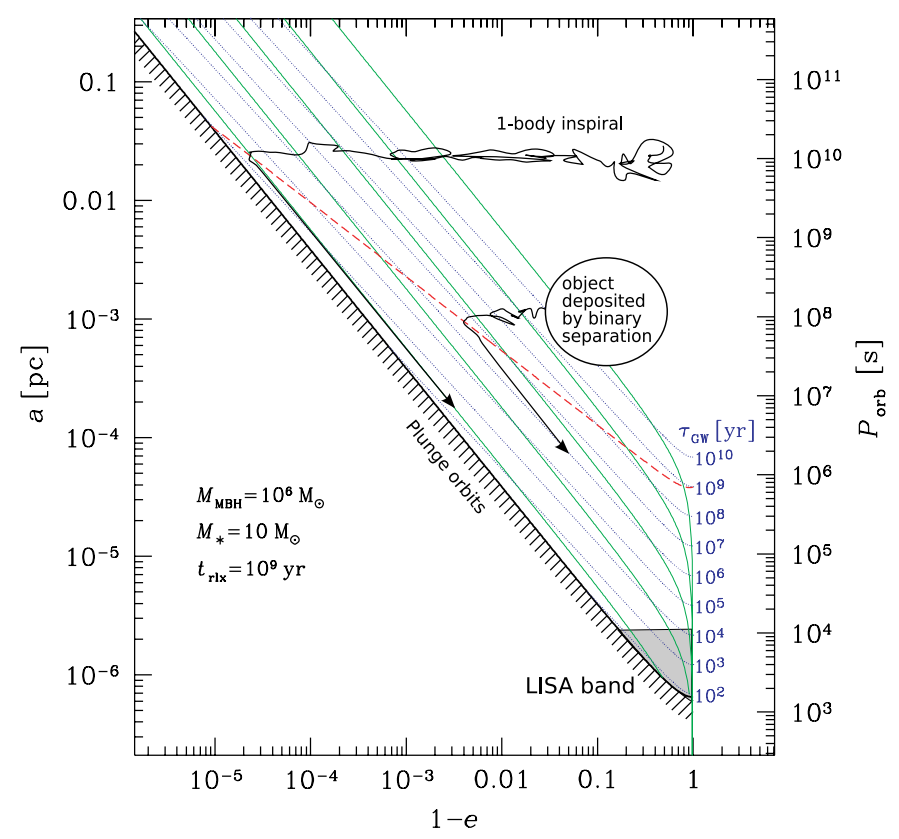

Figure 1. Inspiral trajectories in the semi-major axis, eccentricity plane. The thick diagonal line represents the last stable orbit using effective Keplerian values $\left(R_{\mathrm{p}} \simeq 4 R_{\mathrm{S}}\right.$ for $e \ll 0.1$, see [70] for the general relation). The thin diagonal lines (in green) show inspiral trajectories due to emission of gravitational waves (GWs) and thin dotted (blue) lines are contours of constant time left until plunge, $\tau_{\mathrm{GW}}$, as labelled in years on the right [253]. We assume a $10 M_{\odot}$ stellar black hole orbiting a $10^{6} M_{\odot} \mathrm{MBH}$ on a slowly evolving Keplerian ellipse. The thick (red) dash-dotted line shows $\tilde{e}(a)$, defined by $t_{e}=\tau_{\mathrm{GW}}$ (equation (4) with $C_{\mathrm{EMRI}}=1$ ) assuming a constant value $t_{\mathrm{rlx}}=1 \mathrm{Gyr}$. Below this line, the effects of relaxation on the orbit are negligible in comparison to emission of GWs. We schematically show typical orbital trajectories for EMRIs. Stars captured by tidal binary splitting initially have $a$ of order $100-1000 \mathrm{AU}\left(5 \times\left(10^{-4}-10^{-3}\right) \mathrm{pc}\right)$ and $e=0.9-0.99$ [228]. On a time scale of order $t_{\mathrm{rlx}} \ln (1-\tilde{e})^{-1}$, the eccentricity random-walks into the GW-dominated region, leading to a nearly circular EMRI. If the star has not been deposited by binary splitting but has diffused from large radii or has been captured by GW emission, it will initially have a much larger value of $a$, therefore producing a higher eccentricity EMRI. One sees that stars with $a \lesssim 5 \times 10^{-2}$ pc cannot enter the inspiral domain unless $a$ is first reduced significantly, which takes of order $t_{\mathrm{rlx}}$. The grey region is the domain for sources whose orbital frequency is in the LISA band $P_{\text {orb }}<10^{4} \mathrm{~s}$.

$[36,37,108,163]$, the number of stars per unit $\log (a)$ is roughly $\mathrm{d} \mathcal{N}_{\star} / \mathrm{d}(\log a) \propto a^{(3-\alpha)}$. Also, as one goes inwards, the value of $\alpha$ is lowered by the progressively larger plunge loss cone $[10,195]$. In other words, the stellar density is reduced there (in comparison to a pure power law) because to come and populate this region a star has to spend several relaxation times drifting down in energy while avoiding entering the GW-dominated region and inspiralling quickly.

Implementing this basic scenario in various ways (see section 2.4.5), several authors have estimated the rate at which stellar remnants are captured by the central $\mathrm{MBH}$, with results between $\sim 10^{-6} \mathrm{yr}^{-1}$ and $10^{-8} \mathrm{yr}^{-1}$ for a $10^{6} M_{\odot}$ central black hole $[158,161,171,287]$. When combined with the uncertainty in the number density of massive black holes with $\mathcal{M}_{\bullet}<$ few $\times 10^{6} M_{\odot}$, the net predicted number of detections that LISA will make spans three orders of magnitude, from a few to a few thousand events per year.

We note, incidentally, that even in the LISA band (in the final year of inspiral), the eccentricity of the typical EMRI in the standard picture is high enough that a large number 
of harmonics are likely to contribute to the gravitational waves [26, 104, 161]. In addition, the orbital plane of the EMRIs is unlikely to be significantly correlated with the spin plane of the MBH. These characteristics are distinct from those in non-standard scenarios (discussed below), leading to optimism that some aspects of the nuclear dynamics could be inferred from just a few events.

The word 'capture' is sometimes used to refer to EMRIs, but this is misleading as, in the standard picture, stellar objects are not captured by emission of GWs. They are already bound to the $\mathrm{MBH}$ when they are brought into the GW-dominated regime by two-body relaxation. A star originally unbound to the $\mathrm{MBH}$, with energy $\frac{1}{2} v^{2}$, will be left bound to it by $\mathrm{GW}$ emission if it passes with a pericentre distance smaller than

$$
r_{\text {capt }} \approx 5 R_{\mathrm{S}}\left(\frac{m}{10 M_{\odot}}\right)^{2 / 7}\left(\frac{M_{\odot}}{10^{6} M_{\odot}}\right)^{-2 / 7}\left(\frac{v}{100 \mathrm{~km} \mathrm{~s}^{-1}}\right)^{-4 / 7} .
$$

However, in most cases the star will be left on too wide an orbit. In order to become an EMRI (rather than experience a direct plunge), the semi-major axis has to be smaller than a few $10^{-2}$ pc (see figure 1 and equation (5)), requiring a passage within a distance

$$
r_{\text {capt,EMRI }} \approx 3 R_{\mathrm{S}}\left(\frac{m}{10 M_{\odot}}\right)^{2 / 7}\left(\frac{\mathcal{M}_{\bullet}}{10^{6} M_{\odot}}\right)^{-4 / 7}\left(\frac{a_{\text {capt }}}{0.05 \mathrm{pc}}\right)^{2 / 7}
$$

Therefore for $\mathcal{M}_{\bullet} \ll 10^{6} M_{\odot}$ there is a possibility of capturing unbound (or loosely bound) stars directly onto EMRI orbits. To our knowledge, the contribution of this channel to EMRI rates has not been estimated but is probably small because it is present only for the lowest mass MBHs in the LISA range.

2.1.2. Modifications of the standard picture. There are a number of additional effects now being included in EMRI rate estimates that could alter the rate significantly. In particular, processes that systematically alter the energy distribution of black holes, or change the rate of angular momentum diffusion, could have a substantial impact.

Mass segregation. In galactic nuclei with black holes of mass $\mathcal{M}_{\bullet} \lesssim a 10^{6} M_{\odot}$, relaxation times for stellar-mass black holes are much less than the age of the universe [107, 108, 163, 188, 211]. This suggests that they would have had time to sink in the central potential. Estimates of the effect of this mass segregation indicate that the black holes have time to form a subcluster of radius $\sim 10^{-2} \mathrm{pc}$, which is small enough that their orbits are not perturbed into a rapid plunge [108, 233]. The LISA detection rate is therefore not reduced by plunges.

Triaxiality. Galactic nuclei need not be precisely spherical, particularly if the galaxy has undergone a major merger in the past. Indeed, triaxiality can persist much longer than was once thought, even in the presence of a supermassive black hole [160, 258]. If the potential is non-axisymmetric, it means that an individual black hole need not conserve its angular momentum from orbit to orbit (although of course the overall angular momentum is conserved). This non-conservation means that the pericentre distance can change radically in a dynamical time, and some triaxial potentials favour orbits that are centrophilic. At first sight, it might appear that this effect could increase the LISA detection rate dramatically. However, there are two effects that mitigate this. First, the dominant contributors to the LISA inspiral rate are black holes with semi-major axes of $\sim 10^{-2} \mathrm{pc}$, much smaller than the radius of influence [104, 161]. It is difficult to see how substantial deviations from spherical symmetry could persist in this region. Second, triaxiality implies a greatly enhanced rate of angular momentum diffusion. This implies that the pericentre distance can be reduced significantly in a single 
orbit, but also means that the distance can be increased again in the next orbit. The loss cone for single-passage processes such as tidal disruptions and direct plunges is replenished more efficiently, increasing the rate of such events. However, in the standard picture, EMRIs must occur in the empty loss-cone regime for them to be gradual, i.e., to avoid premature plunge [161]. Although triaxiality might increase the rate of binary tidal separations (see below), what is required to produce more EMRIs with single stellar objects is a process that allows rapid diffusion to small pericentres, but slows down once those pericentres are reached. One such process is resonant relaxation, which we now discuss.

Resonant relaxation. Over times long compared to an orbital time, orbits of different stellarmass black holes affect each other as if they were one-dimensional wires whose mass density at a given radius is proportional to the time spent there. The torques exerted by the wires on each other lead to secular resonances. The integration over many orbits guarantees that the orbital semi-major axes remain constant, but the angular momenta can change substantially [271, 272]. For EMRIs, an important consequence is so-called resonant relaxation, which increases the rate of angular momentum diffusion by a large factor compared to simple twobody scattering. This might seem similar to the effects of triaxiality, but there is a crucial difference. Resonant relaxation is effective because orbital phase relations can be maintained over many orbits. If, however, there is a source of precession, the phase relations are scrambled and angular momentum diffusion is not as effective. In practice this means that if the pericentre distance is large, resonant relaxation operates, but if it is small enough that general relativistic pericentre precession is significant, resonant relaxation is reduced. This is therefore a positive conspiracy: orbits can migrate quickly to low pericentre, but once there they linger. The current best estimates suggest that this could increase the overall LISA detection rate by a factor of a few [162].

2.1.3. Astrophysical uncertainties. Before we proceed to describe alternative scenarios, it is worth stopping to consider the many uncertainties of astrophysical nature which affect EMRI rate estimates, even in the most studied and best understood standard picture.

Number density of massive black holes. The number of galaxies hosting MBHs with a mass in the range $10^{4}-10^{7} M_{\odot}$ is highly uncertain [25] as only three such objects have so far been detected through robust kinematic measurements [90], although there does exist more indirect evidence for their existence based on the activity of low-luminosity active galactic nuclei $[34,35,78,95,135]$. Assuming that all galaxies harbour an $\mathrm{MBH}$ and using the observational distribution of velocity dispersion or spheroid luminosity combined with the $\mathcal{M}_{\bullet}-\sigma$ relation or the correlation between $\mathcal{M}_{\bullet}$ and luminosity [147, 200, 205], one estimates the density of MBHs in the LISA range to be a few $10^{-2} \mathrm{Mpc}^{-3}$ in the local universe [7, 285].

It has been suggested that most small galaxies have a dense stellar cluster at their centre instead of an $\mathrm{MBH}[92,318]$. However, the existence of an $\mathrm{MBH}$ with a mass compatible with the $\mathcal{M}_{0} \propto \sigma^{\beta}$ relation of exponent $\beta \simeq 4$ is only ruled out observationally in the cases of M 33 and NGC 205 [118, 213, 312]. The value of $\beta$ is still not established very accurately and could be as high as 4.5-5 [90], making the upper limit on $\mathcal{M}$ • for NGC 205 compatible with the relation (but shifting the MBH mass function to lower values). It should also be reminded that well-observed galaxies such as the Milky Way, M 31 and M 32 possess both an $\mathrm{MBH}$ of normal mass and a compact stellar nucleus. On the other hand, there are theoretical reasons to suspect a deficiency of low-mass MBHs. Using semi-analytical models for the cosmological assembly of galaxies, one can study statistically the growth and possible ejection of MBHs due to the velocity kick, of up to $\sim 4000 \mathrm{~km} \mathrm{~s}^{-1}$, imparted by asymmetric emission of gravitational waves when two MBHs merge $[22,50]$. These models indicate that, 
in the present-day universe, MBHs might be absent or under-grown in a significant fraction of galaxies with a velocity dispersion below $100 \mathrm{~km} \mathrm{~s}^{-1}$ [315].

For these reasons, it might be wise not to assume the existence of a large population of MBHs with $\mathcal{M}_{\bullet} \lesssim 2 \times 10^{6} M_{\odot}$ for conservative estimates of EMRI detection rates [76]. This uncertainty will probably be decreased by the time LISA flies as present and future observations in the electro-magnetic domain will help constrain the low-mass end of the $\mathrm{MBH}$ population in nearby galaxies, as well as the characteristics of the nuclei in which they reside. For instance, it is predicted that these smaller MBHs should dominate the rate of accretion flares resulting from the tidal disruption of main-sequence or giant stars [317]. Tentative detections of such flares in the UV and x-ray have been reported $[87,121,144,181,182]$ and more robust detections can be expected in the next few years.

Density profiles of galactic nuclei. Direct observational constraints on the stellar density within the sphere of influence of MBHs with masses in the LISA range exist only for the Milky Way [4, 85, 119, 122, 123, 199, 206, 251, 283] and M 32 [188]. Even in these cases the interpretation of the (projected) surface brightness or number counts in terms of stellar density is a difficult and uncertain matter, mostly because these observations are dominated by bright stars whose distribution probably does not trace that of the compact remnants [5]. Furthermore, there is no deep reason why all nuclei harbouring an $\mathrm{MBH}$ of a given mass should have the same stellar density or why there should be scaling laws for the parameters of galactic nuclei with different $\mathcal{M}_{\bullet}$ (the correlations between $\mathcal{M}_{\bullet}$ and the structure of the host galaxy, mentioned above, apply at scales much larger than the influence radius).

Populations of compact remnants in galactic nuclei. In addition to the role of dynamical mass segregation, important unknowns affecting this aspect are (1) the spectrum of masses of newborn stars (known as 'initial mass function' or IMF), (2) the history of stellar formation, i.e., the distribution of ages and metallicities, which strongly affect the populations of compact remnants, and (3) the theory of stellar evolution. There are indications [23, 244, 294] that the IMF in the central regions of our galaxy might be richer in massive stars $\left(M_{*}>5-10 M_{\odot}\right)$ than in other environments $[52,183]$. If this is a generic situation, EMRI rates might be larger due to a higher fractional number of stellar BHs. At the Galactic centre, tens of young massive stars are observed within a few tenths of a pc of the MBH. Their unexplained presence reminds us of how little is understood about stellar formation in galactic nuclei. The prediction of the number, masses and types of compact remnants in galactic nuclei is also hindered by uncertainties in stellar evolution. The mass of candidate stellar BHs is only constrained to be roughly in the range 5-20 $M_{\odot}$ [247], but the formation of lower or higher mass BHs through normal stellar evolution [39] is not excluded. The uncertainties in the theoretical predictions about the relation between the initial mass of a star and the mass and type of remnant produced are still very large [111, 148, 324]. The most recent stellar collapse simulations indicate that, for solar metallicity or above, very few BHs might be produced and these could have typical masses below $5 M_{\odot}$, which would have dire consequences for EMRI rates. The discovery of a neutron star in a young cluster $[236,238]$ recently showed that not all stars more massive than $20-30 M_{\odot}$ become $\mathrm{BHs}$, in contrast to what is commonly assumed.

Before LISA starts operating, we might get a better handle on the population of stellar remnants in our Galactic nucleus through observations and modelling of x-ray point sources [24, 189, 237, 240, 277]. Most of these are probably compact binaries but even single stellar BHs can become bright sources when they cross molecular clouds [75]. However, it is not clear at all how typical the centre of our galaxy is and how this information can be used to constrain models of general nuclei. 
Stellar dynamics around an $M B H$. This aspect is covered in section 2.4.1. In the framework of the (modified) standard scenario, the difficulties are mostly of a numerical nature: how to implement the (gravitational) physics in an efficient and realistic way to obtain robust predictions for EMRIs given an initial galactic nucleus model.

2.1.4. Non-standard scenarios. There are additional ways to place a black hole in a close orbit around an $\mathrm{MBH}$. As mentioned above, the distribution of eccentricities and inclinations in these scenarios differ qualitatively from those in the standard picture.

Binary tidal separation. Suppose that stellar-mass black holes in galactic nuclei are sometimes found in binaries. If such a binary passes close enough to an $\mathrm{MBH}$, one member is typically captured by the MBH while the other is flung out at high speed (indeed, this process is thought to produce hypervelocity stars in the Galaxy; see [47, 84, 136, 155, 157, 159, 256, 323]). The captured black hole is expected to have a semi-major axis of a few hundred AU and a pericentre distance of a few to tens of AU, implying that it will circularize by the time it enters the LISA frequency band [136, 228]. The large allowed pericentre distance means that the cross section for this process is much larger than it is for single-body gravitational wave capture, and if $\gtrsim 1 \%$ of black holes are in binaries, the total rate may compete with the rate from the standard scenario [228]. Note that inspirals of this type are expected to have low eccentricities (see figure 1), but arbitrary inclinations relative to the spin axis of the $\mathrm{MBH}$. As for tidal disruption of single stars [97], in a spherical potential, the largest contribution to the rate of binary separations comes from binaries with a (circum-MBH) semi-major axis of order the "critical radius'. This corresponds to the situation where in just one orbit, relaxation can change the direction of the velocity vector by an angle equal to the aperture of the loss cone. The loss cone is relatively large for binary separation and the critical radius is well outside the radius of influence of the $\mathrm{MBH}$, meaning that the rate can be increased significantly by processes able to replenish loss-cone orbits faster than two-body relaxation on that scale, including massive perturbers such as molecular clouds [252] and the effects of a triaxial potential (see above).

Capture of cores of giants. Giant stars venturing very close to the $\mathrm{MBH}$ have their envelope partially or completely stripped $[74,77]$ by the tidal stresses. It is possible that the dense core of the giant is left on a tight orbit around the MBH and will evolve into an EMRI. However, detailed hydrodynamical calculations are required to determine whether the coupling between the diffuse envelope and the core is strong enough to affect significantly the orbit of the core itself.

Massive star capture or production in disc. Another proposal is related to the presence of massive accretion discs around MBHs. At distances of $\sim 0.1-1 \mathrm{pc}$ from the $\mathrm{MBH}$ and at typical accretion rates, these discs can be unstable to star formation [63, 133, 134, 190-192, $229,243]$. If, as in some calculations, there is a bias towards the production of massive stars in the disc, they could evolve to become black holes, which are then dragged in along with the disc matter. Alternately, massive stars on orbits that cross the disc could be captured and then evolve into black holes [176, 270, 297, 299]. Either way, in the last year of inspiral the holes would be expected to be in nearly circular orbits with orbital planes aligned with the MBH. Rates are highly uncertain as well as the mass of the stellar remnants formed (which could even be IMBHs). However, these events would likely have a different signature waveform than those of the other two classes because they should occur on co-rotating, circular orbits lying in the equatorial plane of the spinning $\mathrm{MBH}$ if it has gained a significant fraction of its mass by accreting from the disc $[32,178,316]$. Moreover, there is the exciting possibility that 
in such a scenario the compact object would open a gap in the disc, which could lead to an optical counterpart to the EMRI event [191].

\subsection{Intermediate-mass black holes and inspirals into $\mathrm{MBHs}$}

The existence of stellar-mass black holes $\left(\sim 5-20 M_{\odot}\right)$ and supermassive black holes $\left(\sim 10^{6-10} M_{\odot}\right)$ has been confirmed via the dynamics of stars around them $([90,247]$ for recent reviews). In contrast, intermediate-mass black holes (IMBHs; $\sim 10^{2-4} M_{\odot}$ ) have not been dynamically confirmed to exist, because they are rare enough that none are known in binaries in the local group, and their radii of influence are small enough to make mapping of stellar motions around them very challenging. The current evidence for them is thus indirect, with the best cases provided by the ultra-luminous X-ray sources (ULXs), which are bright $>10^{39-40} \mathrm{erg} \mathrm{s}^{-1}$, variable, non-nuclear x-ray sources seen in one out of every few galaxies (see [221, 313] for a review). There are several strong, but not conclusive, pieces of evidence that suggest that at least some ULXs are $M>100 M_{\odot}$, including unusually cool accretion discs [224-226], low-frequency quasi-periodic brightness oscillations [296], x-ray dominance of the multiwavelength spectrum (note that if we are instead seeing relativistic jets, we would expect a relatively flat $\nu F_{v}$ spectrum), and surrounding nebular He II luminosity suggesting that the source is quasi-isotropic instead of beamed [248, 249].

In the early metal-free universe, it is possible that individual stars might evolve to several hundred solar mass black holes, where the high mass is a result of less fragmentation during star formation and minimal mass loss to winds and instabilities. In the current universe, the dynamics of dense stellar regions may allow runaway collisions of massive stars in young clusters, with subsequent evolution to an IMBH [83, 100, 109, 110, 139, 140, 246, 262, 263], potentially followed by slower growth of an IMBH via collisionless interactions and mergers with stellar-mass compact objects [137, 138, 222, 223, 234, 235, 246, 303].

Such clusters, if formed within a few hundred parsecs of the galactic nucleus, will sink towards the centre due to dynamical friction. When the cluster is within a few parsecs, the stars are stripped away by the galactic tidal field, and the IMBH continues sinking on its own. There are many uncertainties about the further development, but analytic [227] and subsequent numerical [207, 264] calculations suggest that the resulting IMBH-MBH mergers should be detectable with LISA between a few and a few tens of times per year. As discussed in the data analysis section below, even a single such event would be strong enough to allow unique tests of the predictions of general relativity in strong gravity. It would also shed light on the elusive IMBHs themselves, as well as yielding insight into the dynamics of stellar clusters and galactic nuclei.

\subsection{Expected eccentricity distributions}

2.3.1. Orbital evolution due to emission of gravitational waves. Consider a binary with component masses $m_{1}$ and $m_{2}$, which thus has total mass $M=m_{1}+m_{2}$ and reduced mass $\mu=m_{1} m_{2} / M$. Suppose that its semi-major axis is $a$ and eccentricity is $e$. The Peters equations for gravitational wave emission from a Keplerian orbit [253] give

$$
\left\langle\frac{\mathrm{d} a}{\mathrm{~d} t}\right\rangle=-\frac{64}{5} \frac{G^{3} \mu M^{2}}{c^{5} a^{3}\left(1-e^{2}\right)^{7 / 2}}\left(1+\frac{73}{24} e^{2}+\frac{37}{96} e^{4}\right)
$$

and

$$
\left\langle\frac{\mathrm{d} e}{\mathrm{~d} t}\right\rangle=-\frac{304}{15} e \frac{G^{3} \mu M^{2}}{c^{5} a^{4}\left(1-e^{2}\right)^{5 / 2}}\left(1+\frac{121}{304} e^{2}\right) .
$$


We note that the Peters formalism does not capture the orbital evolution in the strong-field regime, before plunge. In particular, for EMRIs around a spinning $\mathrm{MBH}$, a slight increase in eccentricity might occur in the late evolution [113]. This does not affect the present discussion. From equation (10), the characteristic time to change the eccentricity is

$$
\tau_{\mathrm{GW}}=\frac{e}{|\mathrm{~d} e / \mathrm{d} t|} \approx \frac{15}{304} \frac{c^{5} a^{4}\left(1-e^{2}\right)^{5 / 2}}{G^{3} \mu M^{2}} \approx 8 \times 10^{17} \mathrm{yr}\left(\frac{M_{\odot}}{\mu}\right)\left(\frac{M_{\odot}}{M}\right)^{2}\left(\frac{a}{1 \mathrm{AU}}\right)^{4}\left(1-e^{2}\right)^{5 / 2} .
$$

Here we neglect the near-unity factor $\left(1+121 e^{2} / 304\right)$.

We can rewrite this in terms of gravitational wave frequency. Let us consider in particular the frequency emitted at pericentre. If the orbit is substantially eccentric, then the orbital frequency at that point will be approximately $\sqrt{2}$ times the circular frequency at that radius (because the speed is $\sqrt{2}$ times greater than a circular orbit). If we designate a maximum gravitational wave frequency $f_{\max }$ to be double the frequency at pericentre, then

$$
f_{\max } \approx \frac{1}{\pi}\left[\frac{2 G M}{(a(1-e))^{3}}\right]^{1 / 2}
$$

Therefore

$$
a^{4}=0.75 \mathrm{AU}^{4}\left(\frac{M}{10^{6} M_{\odot}}\right)^{4 / 3}\left(\frac{f_{\max }}{10^{-4} \mathrm{~Hz}}\right)^{-8 / 3}(1-e)^{-4}
$$

and

$$
\begin{aligned}
\tau_{\mathrm{GW}} & \approx 6 \times 10^{2} \mathrm{yr}\left(\frac{\mu}{10^{3} M_{\odot}}\right)^{-1}\left(\frac{M}{10^{6} M_{\odot}}\right)^{-2 / 3}\left(\frac{f_{\max }}{10^{-4} \mathrm{~Hz}}\right)^{-8 / 3}(1+e)^{5 / 2}(1-e)^{-3 / 2} \\
& \approx 3 \times 10^{3} \mathrm{yr}\left(\frac{\mu}{10^{3} M_{\odot}}\right)^{-1}\left(\frac{M}{10^{6} M_{\odot}}\right)^{-2 / 3}\left(\frac{f_{\max }}{10^{-4} \mathrm{~Hz}}\right)^{-8 / 3}(1-e)^{-3 / 2},
\end{aligned}
$$

where in the last line we assume a relatively high eccentricity, so that $1+e \approx 2$.

2.3.2. Eccentricity of EMRIs. A classic EMRI, with $M=10^{4}-10^{7} M_{\odot}$ and $\mu=1-10 M_{\odot}$, could have a significant eccentricity if (as expected in galactic nuclei) the orbits come in from large distances, $a>10^{-2}$ pc with $e \gtrsim 0.9999$. Hopman and Alexander [161] made an estimate of the distribution of eccentricities for one-body inspiral and their results showed that it is skewed to high- $e$ values, with a peak of the distribution at $e \sim 0.7$, at an orbital period of $10^{4} \mathrm{~s}$. On the other hand, following a binary separation event (and possibly the tidal capture of giant's core), the compact star is deposited on an orbit with a semi-major axis of order a few tens to a few hundreds of AU. In this case, the GW-dominated regime is reached with an eccentricity smaller than 0.99 and the orbit should be very close to circular when it has shrunk into LISA band. Such typical orbital evolutions for EMRIs are shown in figure 1.

2.3.3. Eccentricity of IMRIs. In contrast with stellar binaries, it seems likely that the majority of mergers between two massive or intermediate-mass black holes occur because the black holes form separately, then are brought together by dynamical processes. In particular the favoured scenario for IMRIs involves the formation of an IMBH in stellar cluster which spirals into a galactic centre where the cluster is tidally disrupted and deposits the IMBH on an orbit around the central supermassive black hole.

The result is that mergers between massive black holes very probably require substantial dynamical interactions as opposed to pure evolution by emission of gravitational radiation. These dynamical effects can change the eccentricity. On their own, they would tend to make 
the probability distribution 'thermal', i.e., $P(e)=2 e$, which has a mean of $e \approx 0.7$. A bias towards higher eccentricities could happen if capture by emission of gravitational radiation happens preferentially at large $e$. The actual eccentricity is therefore determined by the competition between dynamical wandering and gravitational wave circularization.

To get an estimate of how circular the MBH-IMBH binary will (as a LISA source), we can compare the time to reduce eccentricity via gravitational radiation with the time to interact with enough stars to change the eccentricity significantly. Obviously, high eccentricity is possible if there is a close hyperbolic orbit, but we consider the much more likely case that the evolution is gradual. Here we consider dynamical scenarios in which the primary interactions are of single objects with the binary of interest; this is therefore relevant to an $\mathrm{MBH}-\mathrm{MBH}$ or IMBH-MBH binary, but not to the classic EMRI scenario in which many low-mass objects interact collectively in the radius of influence of an $\mathrm{MBH}$.

To determine the timescale for dynamical eccentricity changes, we note that the orbital speed at a gravitational wave frequency of $10^{-4} \mathrm{~Hz}$ is rather high; roughly $30000 \mathrm{~km} \mathrm{~s}^{-1}\left[M /\left(10^{6} M_{\odot}\right)\right]^{1 / 3}$ for a circular orbit, for example. This is much greater than the velocity dispersion $\sigma$ in any realistic astrophysical environment. Therefore, the cross section $\Sigma$ for a binary-single encounter with a closest approach $r_{p}$ is

$$
\Sigma=\pi r_{p}^{2}\left[1+2 G M /\left(r_{p} \sigma^{2}\right)\right] \approx \pi r_{p}\left(2 G M / \sigma^{2}\right) .
$$

If we assume that $r_{p}<2 a$ is required for a significant interaction, then

$$
\begin{gathered}
\Sigma=4 \pi a\left(\frac{G M}{\sigma^{2}}\right) \approx 2 \times 10^{32} \mathrm{~cm}^{2}\left(\frac{M}{10^{6} M_{\odot}}\right)^{4 / 3}\left(\frac{f_{\max }}{10^{-4} \mathrm{~Hz}}\right)^{-2 / 3} \\
\times\left(\frac{\sigma}{100 \mathrm{~km} \mathrm{~s}^{-1}}\right)^{-2}(1-e)^{-1} .
\end{gathered}
$$

The timescale for a single interaction is just $\tau_{\text {int }}=1 /(n \Sigma \sigma)$, where $n$ is the number density of objects interacting with the binary. However, note that for the eccentricity or semi-major axis of the binary to be affected significantly, the binary must interact with approximately a mass $\sim M$. If the stars interacting with the binary have average mass $\langle m\rangle$, this means that the time required for dynamical interactions to change the eccentricity is $\tau_{\text {dyn }} \approx(M /\langle m\rangle) \tau_{\text {int }}$. This yields the product $n\langle m\rangle$, which is simply the average mass density $\rho$ of things interacting with the binary, so only this quantity matters and we have

$$
\tau_{\mathrm{dyn}} \approx 5 \times 10^{8} \mathrm{yr}\left(\frac{\rho}{10^{6} M_{\odot} \mathrm{pc}^{-3}}\right)^{-1}\left(\frac{M}{10^{6} M_{\odot}}\right)^{-1 / 3}\left(\frac{f_{\max }}{10^{-4} \mathrm{~Hz}}\right)^{2 / 3}\left(\frac{\sigma}{100 \mathrm{~km} \mathrm{~s}^{-1}}\right)(1-e) .
$$

The ratio between the circularization time and the time for dynamical change is therefore

$$
\begin{aligned}
\frac{\tau_{\mathrm{GW}}}{\tau_{\mathrm{dyn}}} \approx 6 \times 10^{-6} & \left(\frac{\mu}{10^{6} M_{\odot}}\right)^{-1}\left(\frac{M}{10^{3} M_{\odot}}\right)^{-1 / 3} \\
& \times\left(\frac{\rho}{10^{6} M_{\odot} \mathrm{pc}^{-3}}\right)\left(\frac{\sigma}{100 \mathrm{~km} \mathrm{~s}^{-1}}\right)^{-1}\left(\frac{f_{\max }}{10^{-4} \mathrm{~Hz}}\right)^{-10 / 3}(1-e)^{-5 / 2} .
\end{aligned}
$$

If $\tau_{\mathrm{GW}} / \tau_{\mathrm{dyn}} \ll 1$ we conclude that circularization dominates; if the ratio is the other way, then circularization is ineffective and the eccentricity will sample a thermal distribution. Therefore, a $10^{3} M_{\odot}$ black hole spiralling into a $10^{6} M_{\odot}$ black hole cannot maintain a significant eccentricity above $\sim 10^{-5} \mathrm{~Hz}$ as its evolution is likely to be strongly dominated by gravitational radiation. However, a slightly eccentric source will exhibit a tell-tale phase evolution which could permit the measurement of eccentricities as low as $\sim 0.01[11,69]$. 


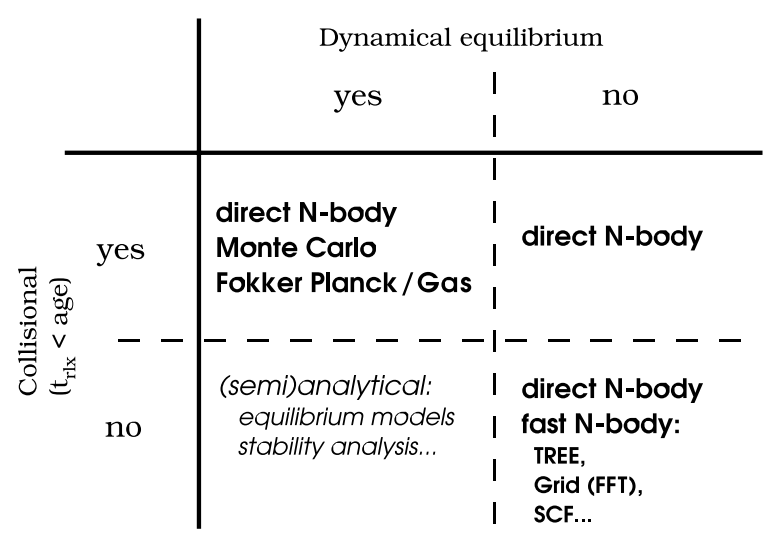

Figure 2. Methods appropriate to the various realms of stellar dynamics.

\subsection{Numerical stellar dynamics for I/EMRIs}

2.4.1. Stellar dynamics for galactic nuclei. We can approximately classify the different kinds of techniques employed for studying stellar dynamics according to the dynamical regime(s) they can cope with. In figure 2 we have a classification of these techniques. (Semi-)analytical methods are generally sufficient only to study systems which are in dynamical equilibrium and which are not affected by collisional (relaxational) processes. In all other cases, including those of importance for EMRI studies, the complications that arise if we want to extend the analysis to more complex (realistic) situations, force us to resort to numerical techniques.

The $N$-body codes are the most straightforward approach from a conceptual point of view. In those, one seeks to integrate the orbital motion of $N$ particles interacting gravitationally. It is necessary to distinguish between the direct $N$-body approaches which are extremely accurate but slow and the fast $N$-body approaches, which are fast but inaccurate and therefore generally deemed inadequate for studying relaxing systems because relaxation is the cumulative effect of small perturbations of the overall, smooth, gravitational potential. Fast $N$-body codes are usually based on either TREE algorithms [33] or on an fast Fourier transform (FFT) convolution to calculate the gravitational potential and force for each particle [89] or on an self-consistentfield (SCF) $[58,153]$ approach. We will not describe these numerical techniques in this section because they have never been used to study E/IMRIs and the approximations on which they are based make them unsuitable for an accurate study of such systems, since relaxation plays a role of paramount importance. Fast $N$-body algorithms can only be employed in situations in which relaxation is not relevant or over relatively short dynamical times, such as in studying bulk dynamics of whole galaxies.

On the other hand, if we want to study a system including both collisional effects and dynamical equilibrium, we can employ direct $N$-body codes or use faster approaches, like the Monte Carlo, Fokker-Planck and gas methods, which we will describe below. The only technique that can cope with all physical inputs is the direct $N$-body approach, in which we make no strong assumptions other than that gravity is Newtonian gravity (although nowadays post-Newtonian corrections have also been incorporated, see section 2.4.6).

If we neglect capture processes driven by tidal effects, the region from where we expect most EMRIs to come from is limited to $\sim 0.1 \mathrm{pc}$ around the central $\mathrm{MBH}$. In that zone the potential is totally spherical. Non-spherical structures such as triaxial bulges or stellar discs are common on scales of 100-1000 pc, and the nucleus itself may be non-spherical. 


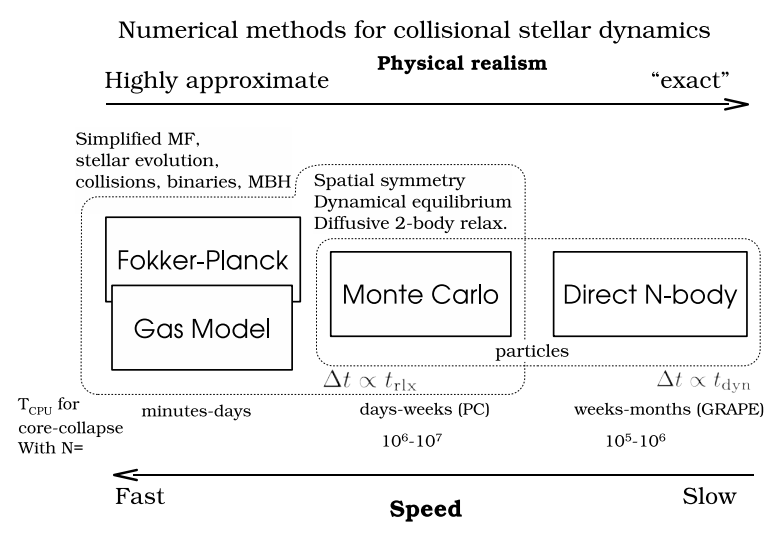

Figure 3. The various methods used to study collisional stellar dynamics.

For example, it could be rotating, as a result of a merger with another nucleus [230] or due to dissipative interactions between the stars and a dense accretion disc [270]. It has been proved that this triaxiality could boost the disruption rates by orders of magnitude $[215,259]$. Whilst assuming sphericity will probably not have any impact on the estimate of capture rates, it is of big relevance for 'tidal processes', since this is the region in which binary tidal separation and the tidal capture of giant cores will happen (see section 2). For these processes the critical radius is beyond the influence radius of the central $\mathrm{MBH}$ and so triaxiality can probably play an important role. Due to insufficient computer power and the limitations of simulation codes galactic nuclei have so far been modelled only as isolated spherical clusters with purely Newtonian gravity (i.e. [106, 241]). More realistic situations could only be explored with $N$-body methods or possibly with hybrid codes (Monte Carlo combined with $N$-body, for instance).

In figure 3 we show a schematic illustration of the current available codes for stellar dynamics including relaxation. From the left to the right the physical realism of the codes increases while the speed decreases. The two-dimensional numerical direct solutions of the Fokker-Planck equation [300-302] probably require the least computational time, but these are followed closely by the gaseous model. The advantage of these two codes are the computational time required to perform a simulation (typically of the order of one minute on a regular PC for a Hubble time) and since they are not particle-based, the resolution can be envisaged as infinite, so that they are not limited by the particle number of the system and there is practically no numerical noise. Nevertheless, although they should be envisaged as powerful tools to make an initial, fast exploration of the parameter space, the results give us tendencies of the system, rather than an accurate answer [9]. Studying the Astrophysical I/EMRI problem requires a meticulous characterization of the orbital parameters, so that approximate techniques should be regarded as exploratory only [76].

2.4.2. The Fokker-Planck approach. Instead of tracking the individual motion of a large number of particles, as in $N$-body methods, one can attempt to describe a system consisting of a very large number of stars through the one-particle phase-space distribution function (DF for short) $f(\vec{x}, \vec{v}, t)$. The best interpretation of $f$ is as a probability density if it is normalized to $1-f(\vec{x}, \vec{v}, t) \mathrm{d}^{3} x \mathrm{~d}^{3} v$ is the probability of finding, at time $t$, any given particle within a volume of phase space $\mathrm{d}^{3} x \mathrm{~d}^{3} v$ around the $6 \mathrm{D}$ phase-space point $(\vec{x}, \vec{v})$; the average number of particles in this volume would be $\mathcal{N}_{\star} f(\vec{x}, \vec{v}, t) \mathrm{d}^{3} x \mathrm{~d}^{3} v$. If the particles move in a 
common smooth potential $\Phi$, the evolution of $f$ is described by the collisionless Boltzmann equation [43]:

$$
D_{t} f \equiv \frac{\partial f}{\partial t}+\vec{v} \cdot \vec{\nabla} f-\vec{\nabla} \Phi \cdot \frac{\partial f}{\partial \vec{v}}=0
$$

$\Phi$ is obtained from $f$ and a possible external potential $\Phi_{\text {ext }}$ (such as produced by a central $\mathrm{MBH})$ from the Poisson equation.

In a real self-gravitating $N$-particle system the potential cannot be smooth on small scales but has some graininess, i.e., short-term, small-scale fluctuations, $\Phi_{\text {real }}=\Phi+\Delta \Phi_{\text {grainy }}$. Relaxation describes the effects of these fluctuations on $f$. They arise because a given particle sees the rest of the system as a collection of point masses rather than as a smooth mass distribution. Relaxational effects, also known (somewhat confusingly) as collisional effects, can therefore be seen as particles influencing each other individually as opposed as to collectively. To allow for these effects, a collision term has to be introduced on the right-hand side of the Boltzmann equation.

The Fokker-Planck (FP) equation is derived by assuming that relaxation is due to a large number of two-body gravitational encounters, each of which leads to a small deflection and occurs 'locally', i.e., these affect the velocity of a star without affecting its position. This is the basis for Chandrasekhar's theory of relaxation [43, 54, 289]. Under these assumptions, one can write

$$
\left.D_{t} f=-\sum_{i=1}^{3} \frac{\partial v_{i}}{\partial[} f(\vec{x}, \vec{v})\left\langle\Delta v_{i}\right\rangle\right]+\frac{1}{2} \sum_{i, j=1}^{3} \frac{\partial^{2}}{\partial v_{i} \partial v_{j}}\left[f(\vec{x}, \vec{v})\left\langle\Delta v_{i} \Delta v_{j}\right\rangle\right],
$$

where the 'diffusion coefficient' $\left\langle\Delta v_{i}\right\rangle$ is the average change in $v_{i}$ per unit of time due to encounters (see $[43,275]$ for a derivation).

From Jeans' theorem [172, 210], for a spherical system in dynamical equilibrium, the DF $f$ can depend on the phase-space coordinates $(\vec{x}, \vec{v})$ only through the (specific) orbital binding energy $E$ and angular momentum (in modulus) $J$,

$$
f(\vec{x}, \vec{v})=F(E(\vec{x}, \vec{v}), J(\vec{x}, \vec{v})) .
$$

In the vast majority of applications, the Fokker-Planck formalism is applied in the twodimensional $(E, J)$-space or, assuming isotropy, the one-dimensional $E$-space rather than the six-dimensional phase space, through the operation of 'orbit averaging' (see [59-61, 289] amongst others).

A standard form of the FP equation for an isotropic, spherical system is

$$
D_{t} N(E) \equiv \frac{\partial N}{\partial t}+\left.\frac{\partial N}{\partial E} \frac{\mathrm{d} E}{\mathrm{~d} t}\right|_{\phi}=-\frac{\partial \mathcal{F}_{E}}{\partial E}
$$

where

$$
\mathcal{F}_{E}=m \mathcal{D}_{E} F-\mathcal{D}_{E E} \frac{\partial F}{\partial E}
$$

is the flux of particles in the energy space; $\mathrm{d} E /\left.\mathrm{d} t\right|_{\phi}$ is the change of energy due to the evolution of the potential $\phi ; N(E)$ is the density of stars in $E$-space

$$
N(E)=16 \pi^{2} p(E) F(E),
$$

with $p(E)=\int_{0}^{r_{\max }} r^{2} v \mathrm{~d} r$. The 'flux coefficients' are

$$
\begin{aligned}
& \mathcal{D}_{E}=16 \pi^{3} \lambda m_{\mathrm{f}} \int_{\phi(0)}^{E} \mathrm{~d} E^{\prime} p\left(E^{\prime}\right) F_{\mathrm{f}}\left(E^{\prime}\right), \\
& \mathcal{D}_{E E}=16 \pi^{3} \lambda m_{\mathrm{f}}^{2}\left[q(E) \int_{E}^{0} \mathrm{~d} E^{\prime} F_{\mathrm{f}}\left(E^{\prime}\right)+\int_{\phi(0)}^{E} \mathrm{~d} E^{\prime} q\left(E^{\prime}\right) F_{\mathrm{f}}\left(E^{\prime}\right)\right],
\end{aligned}
$$


where $\lambda \equiv 4 \pi G^{2} \ln \Lambda . q(E)=\frac{1}{3} \int_{0}^{r_{\max }} r^{2} v^{3} \mathrm{~d} r$ is the volume of phase space accessible to particles with energies lower than $E$ and $p(E)=\partial q / \partial E$ [132].

We used an index ' $f$ ' for 'field' to distinguish the mass and DF of the population we follow ('test-stars') from the 'field' objects. This distinction does not apply to a single-component system but it is easy to generalize to a multi-component situation by summing over components to get the total flux coefficient

$$
\mathcal{D}_{E}=\sum_{l=1}^{N_{\text {comp }}} \mathcal{D}_{E, l}, \quad \mathcal{D}_{E E}=\sum_{l=1}^{N_{\text {comp }}} \mathcal{D}_{E E, l},
$$

where the flux coefficient for component $l$ can be written by replacing the subscript ' $\mathrm{f}$ ' by ' $l$ ' in equation (25).

We now explain schematically how the FP equation is implemented numerically to follow the evolution of star clusters. A more detailed description can be found in, e.g., Chernoff and Weinberg [56]. In the most common scheme, pioneered by Cohn [60], two types of steps are employed alternately, a method known as 'operator splitting'.

(1) Diffusion step. The change in the distribution function $F$ for a discrete time-step $\Delta t$ is computed by use of the FP equation assuming the potential $\phi$ is fixed, i.e., setting $D_{t} N=\partial N / \partial t=\partial N /\left.\partial t\right|_{\text {coll }}$. The FP equation is discretized on an energy grid. The flux coefficients are computed using the $\mathrm{DF}(\mathrm{s})$ of the previous step; this makes the equations linear in the values of $F$ on the grid points. The finite-differencing scheme is the implicit Chang and Cooper [55] algorithm, which is first order in time and energy.

(2) Poisson step. Now the change of potential, resulting from the modification in the DF, $F$ is computed and $F$ is modified to account for the term $\mathrm{d} E /\left.\mathrm{d} t\right|_{\phi}$, i.e., assuming $D_{t} N=\partial N / \partial t+\partial N / \partial E \mathrm{~d} E /\left.\mathrm{d} t\right|_{\phi}=0$. This can be done implicitly because, as long as the change in $\phi$ over $\Delta t$ is very small, the actions of each orbit are adiabatic invariants. Hence, during the Poisson step, the distribution function, expressed in terms of the actions, does not change. In practice, an iterative scheme is used to compute the modified potential, determined implicitly by the modified DF, through the Poisson equation. The iteration starts with the values of $\phi, \rho$, etc computed before the previous diffusion step.

A variant of the FP equation analogous to equation (22) can be written which allows for anisotropy by taking into account the dependence of $F$ on $J$ and including a $J$-flux and corresponding flux coefficients [59, 61, 62, 81, 300-302]. The expressions for the flux coefficients are significantly longer than in the isotropic case and we do not present them here. However, we note that in galactic nuclei, in contrast to globular clusters, anisotropy plays a key role because of the existence of a loss cone.

The use of the FP approach to determine the distribution of stars around an MBH requires a few modifications. First the (Keplerian) contribution of the MBH to the potential has to be added. Several authors have made use of the FP or similar formalisms to study the dynamics well within the influence radius under the assumption of a fixed potential $[19,20,62,162,163$, $195,214]$, which is a significant simplification. The static potential included a contribution for the stellar nucleus in the last study [214] but was limited to a Keplerian MBH potential in the other cases. The presence of the MBH also constitutes a central sink as stars are destroyed or swallowed if they come very close to it. This has to be implemented into FP codes as a boundary condition. Lightman and Shapiro [195] and Cohn and Kulsrud [62] have developed detailed (and rather complex) treatments of the loss cone for the anisotropic FP formalism. It can be used in a simplified way in an isotropic FP analysis [20] to obtain a good approximation to the distribution of stars around an $\mathrm{MBH}$ and of the rates of consumption of stars by the 
MBH. However, additional analysis is required to determine what fraction of the swallowed stars are EMRIs and what their orbital properties are [161, 163].

2.4.3. The gaseous model. Another way to approximately solve the (collisional) Boltzmann equation is to take velocity moments of it. The moment or order $n=0$ of the DF is the density, the moments of order $n=1$ are bulk velocities and $n=2$ corresponds to (anisotropic) pressures (or velocity dispersions). This is analogous to the derivation of the Jeans equation from the collisionless Boltzmann equation [43] but the collision term introduces moments of order $n+1$ in the equations for moments of order $n$. In the gaseous model ${ }^{5}$, one assumes spherical symmetry (but not necessarily dynamical equilibrium) and truncates the infinite set of moment equations at $n=2$. The system is closed with the assumption that energy exchanges between stars through two-body relaxation can be approximated by an ad hoc (local) heat conduction prescription $[143,198]$. This reduces the study of the stellar system to that of a self-gravitating conducting gas sphere. Multi-mass models have been implemented [126, 197, 290, 291] and the detailed forms for the conductivities have been improved by comparing to direct $N$-body models (described below). The addition of a central accreting $\mathrm{MBH}$ and a treatment for loss-cone effects was done by Amaro-Seoane et al [10] for the single-mass case (a comprehensive description of the code is in the appendix of the same work), and by Amaro-Seoane [9] for a stellar mass spectrum.

2.4.4. Hénon-type Monte Carlo codes. The Monte Carlo (MC) numerical scheme is intermediate in realism and numerical efficiency between Fokker-Planck or gas approaches, which are very fast but based on a significantly idealized description of the stellar system, and direct $N$-body codes, which treat (Newtonian) gravity in an essentially assumption-free way but are extremely demanding in terms of computing time. The MC scheme was first introduced by Hénon to follow the relaxational evolution of globular clusters [149-152]. To our knowledge there exist three independent codes in active development and use which are based on Hénon's ideas. The first is the one written by Giersz (see [124] for his most recent published work), which implements many of the developments first introduced by Stodołkiewicz [292, 293]. Second is the code written by Joshi $[174,175]$ and greatly improved and extended by Gürkan and Fregeau (see for instance [98, 99, 139, 140]). These codes have been applied to the study of globular and young clusters. Finally, Freitag developed an MC code specifically aimed at the study of galactic nuclei containing a central MBH $[105,106,108]$. The description of the method given here is based on this particular implementation.

The MC technique assumes that the cluster is spherically symmetric and represents it as a set of particles, each of which may be considered as a homogeneous spherical shell of stars sharing the same orbital and stellar properties. The number of particles may be lower than the number of stars in the simulated cluster but the number of stars per particle has to be the same for each particle. Another important assumption is that the system is always in dynamical equilibrium so that orbital time scales need not be resolved and the natural time-step is a fraction of the relaxation (or collision) time. Instead of being determined by integration of its orbit, the position of a particle (i.e., the radius $R$ of the shell) is picked up at random, with a probability density for $R$ that reflects the time spent at that radius: $\mathrm{d} P / \mathrm{d} R \propto 1 / V_{\mathrm{r}}(R)$ where $V_{\mathrm{r}}$ is the radial velocity. The Freitag scheme adopts time steps that are some small fraction $f$ of the local relaxation (or collision) time: $\delta t(R) \simeq f_{\delta t}\left(t_{\mathrm{rlx}}^{-1}+t_{\text {coll }}^{-1}\right)^{-1}$. Consequently, the central parts of the cluster, where evolution is faster, are updated much more frequently than the outer parts. At each step, a pair of neighbouring particles is selected randomly with

\footnotetext{
5 http://www.ari.uni-heidelberg.de/gaseous-model.
} 
probability $P_{\text {selec }} \propto 1 / \delta t(R)$. This ensures that a particle stays for an average time $\delta t(R)$ at $R$ before being updated.

Relaxation is treated as a diffusive process, using the classical Chandrasekhar theory on which FP codes are also based. The long-term effects on orbits of the departure of the gravitational field from a smooth stationary potential are assumed to arise from a large number of uncorrelated, small angle, hyperbolic two-body encounters. If a star of mass $M_{1}$ travels with relative velocity $v_{\text {rel }}$ through a homogeneous field of stars of mass $M_{2}$ with number density $n$ for a time $\delta t$, then in the centre-of-mass reference frame, its trajectory will be deflected by an angle $\theta_{\delta t}$ with average values

$$
\left\langle\theta_{\delta t}\right\rangle=0 \quad \text { and } \quad\left\langle\theta_{\delta t}^{2}\right\rangle=8 \pi \ln \Lambda G^{2} n\left(M_{1}+M_{2}\right)^{2} \delta t,
$$

where $G$ is the gravitational constant and $\ln \Lambda \simeq 10-15$ is the Coulomb logarithm. In the MC code, at each step, the velocities of the particles of the selected pair are modified as if by a hyperbolic encounter with deflection angle $\theta_{\text {eff }}=\sqrt{\left\langle\theta_{\delta t}^{2}\right\rangle}$. The particles are then put at random positions on the slightly modified orbits. As a given particle will be selected many times, at various positions on its orbit, the MC scheme will integrate the effect of relaxation over the particle's orbit and over all possible field particles. Proper averaging is ensured if the time steps are sufficiently short for the orbit to be modified significantly only after a large number of effective encounters. The energy is trivially conserved to machine accuracy in such a scheme because the same deflection angle $\theta_{\text {eff }}$ is applied to both particles in an interacting pair. Only the direction of the relative velocity vector is changed by $\theta_{\text {eff }}$.

Using a binary tree structure which allows quick determination and updating of the potential created by the particles, the self gravity of the stellar cluster is included accurately. This potential is not completely smooth because the particles are infinitesimally thin spherical shells whose radii change discontinuously. Test computations have been used to verify that the additional, unwanted, relaxation is negligible provided the number of particles is larger than a few tens of thousands.

Although Hénon's method is based on the assumption than all departures from the smooth potential can be treated as two-body small angle scatterings, it is flexible enough to incorporate more realism. The dynamical effect of binaries (i.e., the dominant three- and four-body processes), which may be crucial in the evolution of globular clusters, has been included in various MC codes through the use of approximate analytical cross-sections [127, 269, 293]. Recently, Fregeau [99, 140] has introduced a much more realistic treatment of binaries by on the fly, explicit integrations of the three- or four-body interactions, a brute force approach that is necessary to deal with the full diversity of unequal-mass binary interactions. This approach was pioneered by Giersz and Spurzem [128] in a hybrid code where binaries are followed as MC particles while single stars are treated as a gaseous component.

The few two-body encounters that lead to large angle $(>\pi / 10$, say) deflections are usually neglected. In globular clusters, these 'kicks' have a negligible imprint on the overall dynamics $[132,152]$ but it has been suggested that they lead to a high ejection rate from the density cusp around a central (I)MBH [196]. Kicks can be introduced in the MC code, where they are treated in a way similar to collisions, with a cross section $\pi b_{\text {l.a. }}^{2}$, where $b_{\text {l.a. }}=f_{\text {l.a. }} G\left(M_{1}+M_{2}\right) v_{\text {rel }}^{-2}$. $f_{\text {l.a. }}$ is a numerical factor to distinguish between kicks and 'normal' small angle scatterings (impact parameter $>b_{\text {l.a. }}$ ). However, recent simulations seem to indicate that such kicks have little influence on the evolution of a stellar cusp around an MBH [108].

The MC code is much faster than a direct $N$-body integration: a simulation of a MilkyWay-type galactic nucleus represented by $10^{7}$ particles requires between a few days and a few weeks of computation on a single CPU. Furthermore, with the proper scaling with the number of stars, the number of stars represented is independent of the number of particles. A high 
particle number is obviously desirable for robust statistics, particularly when it comes to rare events such as star-MBH interactions. In contrast, because they treat gravitational (Newtonian) interactions on a elementary level, without relying on any theory about their collective and/or long-term effects, the results of direct $N$-body codes can generally be applied only to systems with a number of stars equal to the number of particles used.

2.4.5. Applications of Monte Carlo and Fokker-Planck simulations to the EMRI problem. $\mathrm{MC}$ and FP codes are only appropriate for studying how collisional effects (principally relaxation) affect spherical systems in dynamical equilibrium. These assumptions are probably valid within the radius of influence of MBHs with masses in the LISA range. Indeed, assuming naively that the $\mathrm{Sgr} \mathrm{A}^{*}$ cluster at the centre of our Galaxy is typical (as far as the total stellar mass and density is concerned) and that one can scale to other galactic nuclei using the $M-\sigma$ relation in the form $\sigma=\sigma_{\mathrm{MW}}\left(\mathcal{M}_{\bullet} / 3.6 \times 10^{6} M_{\odot}\right)^{1 / \beta}$ with $\beta \approx 4-5$ [91, 308], one can estimate the relaxation time at the radius of influence to be $t_{\mathrm{rlx}}\left(R_{\text {infl }}\right) \approx 25 \times 10^{9} \mathrm{yr}\left(\mathcal{M}_{\bullet} / 3.6 \times 10^{6} M_{\odot}\right)^{(2-3 / \beta)}$.

Although observations suggest a large spread amongst the values of the relaxation time at the influence radius of MBHs with similar mass (see, e.g., figure 4 of [214]), most galactic nuclei hosting MBHs less massive than a few $10^{6} M_{\odot}$ are probably relaxed and amenable to $\mathrm{MC}$ or FP treatment. Even if the age of the system is significantly smaller than its relaxation time, such approaches are valid as long as the nucleus is in dynamical equilibrium, with a smooth, spherical distribution of matter. In such conditions, relaxational processes are still controlling the EMRI rate, no matter how long the relaxation time is, but one cannot assume a steady-state rate of diffusion of stars onto orbits with small pericentres, as is often done in FP codes (see the discussion in [231], in the different context of the evolution of binary MBHs).

The Hénon-type MC scheme has been used by Freitag and collaborators to determine the structure of galactic nuclei [101, 106, 108]. Predictions for the distribution of stars around an $\mathrm{MBH}$ have also been obtained by solving some form of the Fokker-Planck equation $[20,162,163,214,241]$ or using the gaseous model $[9,10]$. These methods have proved useful to determine how relaxation, collisions, large-angle scatterings, $\mathrm{MBH}$ growth, etc, shape the distribution of stars around the $\mathrm{MBH}$, which is an obvious prerequisite for the determination of the rate and characteristics of EMRIs. Of particular importance is the inward segregation of stellar BHs as they lose energy to lighter objects. This effect, combined with the fact that stellar BHs produce GWs with higher amplitude than lower mass stars, explains why they are expected to dominate the EMRI detection rate [163, 287]. An advantage of the MC approach is that it can easily and realistically include a continuous stellar mass spectrum and extra physical ingredients. However, the first point might not be critical here as MC results suggest that, for models where all the stars were born $\sim 10 \mathrm{Gyr}$ ago, the pattern of mass segregation can be well approximated by a population of two components only, one representing the stellar BHs and the other representing all other (lighter) objects [108]. Furthermore, the uncertainties are certainly dominated by our lack of knowledge about where and when stellar formation takes place in galactic nuclei, what the masses of the stars which form might be, and what type of compact remnants they become.

The most recent FP results concerning mass segregation were obtained under the assumptions of a fixed potential and an isotropic velocity dispersion, with the effects of (standard or resonant) relaxation being averaged over $J$ at a given energy. The MC code includes the self-gravity of the cluster so the simulated region can extend past the radius of influence, allowing a more natural outer boundary condition. We note that one has to impose a steeper density drop-off at large radii than what is observed to limit the number of particles to a reasonable value while keeping a good resolution in the region of influence. 
The MC code naturally allows anisotropy and implicitly follows relaxation in both $E$ and $J$. Anisotropic FP codes for spherical self-gravitating systems exist [81, 301, 302] but, to our knowledge, none are currently in use that also include a central $\mathrm{MBH}$. Unique amongst all stellar dynamical codes based on the Chandrasekhar theory of relaxation is FoPAX, a FP code which assumes axial rather than spherical symmetry, thus permitting the study of clusters and nuclei with significant global rotation (see [94] and references therein) and which has recently been adapted to include a central $\mathrm{MBH}$ [93].

Determining the EMRI rates and characteristics is a harder challenge for statistical stellar dynamics codes because these events are intrinsically rare and critically sensitive to rather fine details of the stellar dynamics around an MBH. As we explained in section 2.1.1, the main difficulty, in comparison with, for example, tidal disruptions, is that EMRIs are not 'onepassage' events but must be gradual. The first estimate of EMRI rates was performed by Hils and Bender [158]. Assuming a static cusp profile, they followed the evolution of the orbits of test-particles subject to GW emission (equations (9) and (10)) and two-body relaxation introduced by random perturbations of the energy and angular momentum according to precomputed 'diffusion coefficients'. Hopman and Alexander [161] have used a refined version of this 'single-particle Monte Carlo method', as well as the Fokker-Planck equation, to make a more detailed analysis. It was found that no more than $\sim 10 \%$ of the compact objects swallowed by the MBH are EMRIs, while the rest are direct plunges.

Determination of EMRI rates and characteristics were also attempted with Freitag's MC code [102-104]. Despite its present limitations, this approach might serve to inspire future, more accurate, computations and is therefore worth describing in some detail. The MC code does not include GW emission explicitly (or any other relativistic effects). At the end of the step in which two particles have experienced an encounter (to simulate two-body relaxation), each particle is tested for entry into the 'radiation-dominated' regime, defined by equation (4) (with $C_{\mathrm{EMRI}}=1$ ). A complication arises because the time step $\delta t$ used in the MC code is a fraction $f_{\delta t}=10^{-3}-10^{-2}$ of the local relaxation time $t_{\mathrm{rlx}}(R)$, which is generally much larger than the critical timescale defined by the equality $\tau_{\mathrm{GW}}(e, a)=C_{\mathrm{EMRI}}(1-e) t_{\mathrm{rlx}}$. In other words, the effective diffusion angle $\theta_{\text {eff }}$ is generally much larger than the opening angle of the 'radiation cone', $\tilde{\theta} \equiv(1-\tilde{e})^{1 / 2}$. So that the entry of the particle into the radiation cone (corresponding to a possible EMRI) is not missed, it is assumed that, over $\delta t$, the energy of a given particle does not change. Hence, each time it comes back to a given distance from the centre, its velocity vector has the same modulus but relaxation makes its direction execute a random walk with an individual step per orbital period of $\theta_{\text {orb }}=\theta_{\text {eff }}\left(P_{\text {orb }} / \delta t\right)^{1 / 2}$. Entry into the unstable or radiation cone is tested at each of these sub-steps. If the particle is found on a plunge or radiation-dominated orbit, it is immediately removed from the simulation and its mass is added to the MBH.

Unfortunately, in addition to this approximate way of treating relaxation on small time scales, there are a few reasons why the results of these simulations may be only indicative. One is the way $t_{\mathrm{rlx}}$ is estimated, using the coefficient in front of $\delta t$ in equation (27), i.e., an estimate based on the neighbouring particle. Even if it is correct on average, this estimate is affected by a very high level of statistical noise and its value can be far too long in some cases (e.g., when the relative velocity between the particles in the pair is much larger than the local velocity dispersion). This could lead one to conclude erroneously that a star has reached the radiation-dominated regime and will become an EMRI. To improve on this one could base the $t_{\mathrm{rlx}}$ estimate on more than one point on the orbit and on more than one 'fieldparticle' (the number of stars within a distance of $10^{-2} \mathrm{pc}$ of Sgr $\mathrm{A}^{*}$ is probably larger than 1000 , so $t_{\mathrm{rlx}}$ is a well-defined quantity even at such small scales). Another limitation is that GW emission is not included in the orbital evolution, which forces one to assume an abrupt 
transition when $\tau_{\mathrm{GW}}=(1-e) t_{\mathrm{rlx}}$. Hopman and Alexander [161] have also shown that a value of $C_{\mathrm{EMRI}}$ as small as $10^{-3}$ might be required to be sure the EMRI will be successful. Furthermore, the MC simulations carried out so far suffer from relatively poor resolution, with each particle having the statistical weight of a few tens of stars. To improve this one would need to limit the simulation to a smaller volume (such as the influence region) or develop a parallel implementation of the MC code to use $\sim 10^{8}$ particles. Finally, these MC simulations did not include resonant relaxation, an effect which can increase the EMRI rate by of order 10 or completely suppress it, depending on its strength [162].

Future of statistical stellar-dynamical simulations for EMRIs. Despite recent progress, a full understanding of the stellar dynamical processes leading to EMRIs and a robust prediction for their rate and characteristics (eccentricities, stellar types and masses etc) are still lacking. The key points to address include

- interplay between relaxation and GW emission in the orbital evolution of EMRI 'precursors';

- importance of resonant relaxation;

- dynamics of binaries in a galactic nucleus;

- effects of non-sphericity in the potential.

We now suggest how new or updated statistical stellar-dynamics methods can contribute to clarify these points.

A self-gravitating, multi-mass, anisotropic Fokker-Planck [241] code, including a detailed treatment of the loss-cone [62] and with additional terms to include GW-emission and resonant relaxation [161, 162, 171] could provide EMRI (and direct-plunge) rates while accounting for mass segregation at all scales, from the vicinity of the $\mathrm{MBH}$ to well beyond the radius of influence, thus including the whole volume where relaxational effects might play a role. In contrast to FP codes the gaseous approach does not operate in $(E, J)$ phase space where each point corresponds to a well-defined orbit, but in direct space (i.e., the radial coordinate $R$ ) at each point of which only statistical quantities (velocity dispersions) are used to represent approximately the kinematics of all orbits crossing this position. Therefore the treatment of loss-cone effects can only be done in a much more approximate way $[9,10]$, making it probably unsuitable for determining EMRI rates. The FP codes evolve distribution functions, therefore relying on the assumption that any 'interesting' element of phase space always contains a significant number of stars. It is not clear how to apply them to or interpret their results for situations such as EMRIs, corresponding to rare events where small-number effects might be crucial. They require that the stellar population be discretized into a set of 'components' sharing the same properties ( $E, J$, stellar mass and age, etc) and can therefore not treat accurately a continuous mass function, a mixed-age population, or binaries.

Monte Carlo codes are based on a more direct star-by-star (or particle-by-particle) approach making it much easier to consider realistically complex stellar populations and to follow in detail the evolution of individual orbits. Although single-particle MC computations lack self-consistency, the only application of an Hénon-type code to EMRIs suffered from a few shortcomings connected with insufficient resolution in time or particle number. However, there are different avenues for useful MC work.

- Monte Carlo simulations of binary dynamics. The evolution of binaries in a galactic nucleus is still a virtually unexplored territory whose study is made urgent both by the possibility of forming EMRIs (and hyper-velocity stars) through binary separation and by the observation of X-ray binaries around Sgr A* $[239,240]$. Binary stars can be included in a very realistic way into Hénon-type MC codes, using explicit few-body integrations to 
treat interactions between a binary and another single or binary star $[99,128]$ or between a binary and the central MBH.

- Keplerian Monte Carlo. Barring tidal processes such as binary separation or the capture of the core of a giant, the rate of EMRIs is determined by the stellar dynamics well within the influence radius of the MBH. To follow the dynamics in this region with both accuracy and computational efficiency, the development of a specialized MC code is planned. The potential will be the simple Keplerian contribution of the MBH with no account for self gravity of the stellar cluster besides relaxation. It is expected that, in such an external potential, one does not need to conserve energy exactly as for a self-gravitating cluster. Therefore, each particle (representing just one star) can have its own time step, adapted to its orbit. The effects of GW emission can be taken into account explicitly and the dynamics in or close to the 'radiative' cone can be followed on appropriately short timescales.

- Non-spherical hybrid code. Departure from the assumption of sphericity may be important for two non-standard EMRI channels (see section 2.1.4). The first is the one involving a dense accretion disc. In this case, the potential might still be essentially spherical but dissipative interactions with the disc might cause a flattening of the distribution of stars and a significant rate of equatorial circular EMRI events. The second situation is the contribution of binary tidal separations which (unlike standard EMRIs) can be increased significantly by replenishing the loss cone outside the sphere of influence. At the present time FOPAX is the only statistical stellar-dynamics code which does not rely on the assumption of spherical symmetry. It is, however, limited to axially symmetric systems and a particle-based method would be more suitable for the inclusion of stardisc interaction effects or binaries. Following a suggestion already made by Hénon [149], a collisionless $N$-body code could be used as a 'backbone' orbit integrator and complemented by the pairwise treatment of relaxation of the Monte Carlo method. Sigurdsson has applied a similar idea to the study of globular clusters by combining the self-consistent-field $N$-body algorithm with FP terms for two-body relaxation [173].

Some possibly important aspects of the dynamics cannot be treated directly by statistical methods. Important examples are resonant relaxation or the possible effects of the motion of the MBH ([212] and references therein). These processes require direct $N$-body simulations for their study before they can be introduced in an approximate way into FP or MC codes.

2.4.6. Direct-summation $N$-body codes. We finish by considering the $\operatorname{direct} N$-body approach $[1,2,261]$. This is the most expensive method because it involves integrating all gravitational forces for all particles at every time step, without making any a priori assumptions about the system. The NBODy codes use the improved Hermite integration scheme as described in [1, 2], which requires computation of not only the accelerations but also their time derivatives. Since these approaches integrate Newton's equations directly, all Newtonian gravitational effects are included naturally. More crucial for this subject is that the family of the direct NBODY codes of Aarseth also includes versions in which both $K S$ regularization and chain regularization are employed, so that when particles are tightly bound or their separation becomes too small during a hyperbolic encounter, the system is regularized (as described first in [2, 184]) to prevent dangerous small individual time steps. This means that we can accurately follow and resolve individual orbits in the system. Other schemes which make use of a softening in the gravitational forces (i.e. $1 /\left(r^{2}+\epsilon^{2}\right)$ instead of $1 / r^{2}$, where $\epsilon$ is the softening parameter) cannot be employed because $\epsilon$ can induce unacceptable errors in the calculations. The $N$-body codes scale as $\mathcal{N}_{\star}^{2}$, or $\Delta t \propto t_{\text {dyn }}$, which means that even with special-purpose hardware, a simulation can take of the order of weeks if not months. This hardware is the GRAPE (short for GRAvity PipE), a family of hardware which acts as a Newtonian force accelerator. For instance, a 
GRAPE-6A PCI card has a peak performance of 130 Gflop, roughly equivalent to 100 single PCs [141]. It is possible to parallelize basic versions of the direct NBody codes (without including regularization schemes) on clusters of PCs, each equipped with one GRAPE-6A PCI card. This leads to efficiencies greater than 50\% and speeds in excess of 2 TFlops and thus the possibility of simulating up to $\mathcal{N}_{\star}=2 \times 10^{6}$ stars [146]. Nevertheless, when we consider the situation relevant to an EMRI, in which mass ratios are large and we need to follow thousands of orbits, the Hermite integrator is not suitable and problems show up even in the Newtonian regime. Aarseth et al [2,3] summarize different methods developed to cope with large systems with one or more massive bodies. The problem becomes even more difficult when including relativistic corrections to the forces when the stellar black hole approaches the central $\mathrm{MBH}$, because extremely small time-scales are involved in the integration. Progress is being made in this direction with a recently developed time-transformed leapfrog method [218] (for a description of the leapfrog integrator see [219]) and the even more promising wheel-spoke regularization, which was developed to handle situations in which a very massive object is surrounded by strongly bound particles, precisely the situation for EMRIs [2, 325]. Additionally, one must include post-Newtonian $(\mathcal{P N})$ corrections in the direct NBody code because secular effects such as Kozai or resonant relaxation may be smoothed out significantly by relativistic precession and thus have an impact on the number of captures [162].

There already exists a version of direct NBODY that includes relativistic corrections at $1 \mathcal{P N}, 2 \mathcal{P N}$ (periapsis shifts) and $2.5 \mathcal{P N}$ (energy loss in the form of gravitational wave emission) [185]. The authors included perturbations in the $K S$ regularization scheme, so that the forces (actually the accelerations) were modified by

$$
F=\underbrace{F_{0}}_{\text {Newtonian }}+\underbrace{c_{1 \mathcal{N}}^{c^{-2} F_{2}}+\underbrace{c^{-4} F_{4}}_{2 \mathcal{P N}}}_{\text {periapsis shift }}+\underbrace{c^{-5} F_{5}}_{\mathrm{GW}}+\mathcal{O}\left(c^{-6}\right)
$$

Note that the perturbations do not need to be small compared to the two-body force [217]. The method will be applicable even when the relativistic terms become comparable to the Newtonian term provided the KS time step is duly adjusted. Nonetheless, the gauge choice is crucial, since it can introduce an artificially enlarged cross-section for the pairs if the centre-of-mass of the system is 'wobbling' [73, 288]. The desired frame is the centreof-mass frame, which is equivalent to the centre-of-mass Hamiltonian in the ADM (Arnowit, Deser and Misner) formalism [44]. The relative accelerations then have the form

$$
\frac{\mathrm{d} v^{i}}{\mathrm{~d} t}=-\frac{m}{r^{2}}\left[(1+\mathcal{A}) n^{i}+\mathcal{B} v^{i}\right]+\mathcal{O}\left(\frac{1}{c^{7}}\right),
$$

[44] where the relative separation of the binary components is $x^{i}=y_{1}^{i}-y_{2}^{i}, r=|\mathbf{x}|$ and $n^{i}=x^{i} / r ; \mathcal{A}$ and $\mathcal{B}$ are given by the expressions (3.10a) and (3.10b) of [44]. Whilst the gauge choice was not a problem for the system studied in [185], since they were interested in the global dynamical evolution, for the EMRI problem the centre-of-mass frame (located at the origin of the coordinates) must be employed. The integration cannot be extended to velocities higher than $\sim 0.3 \mathrm{c}$, because at these velocities the post-Newtonian formalism can no longer be applied accurately. This means that we cannot reach the final coalescence of the stellar $\mathrm{BH}$ with the $\mathrm{MBH}$, but nonetheless the parameter space that can be explored is unprecedented in scope. There has also been encouraging progress including $\mathcal{P} \mathcal{N}$ corrections in conjunction with alternative high-accuracy methods [13]. We note that it will not be possible to include in $N$-body codes all the $\mathcal{P N}$ corrections that are required for accurate modelling of the phase evolution of the EMRI during the last few years before plunge. However, the $N$-body codes are not required in that regime, since the system is then decoupled from the rest of the stellar 
cluster. Accurate modelling of this regime is required for detection of these systems, and this will be described in section 3.2 .

\section{EMRI detection}

\subsection{Data analysis algorithms}

A typical EMRI signal will have an instantaneous amplitude an order of magnitude below the LISA's instrumental noise and (at low frequencies) as many as several orders of magnitude below the gravitational wave foreground from Galactic compact binaries. This makes detection a rather difficult problem. However, the signals are very long lived, and will be observed over more than $10^{5}$ cycles, which in principle allows the signal-to-noise ratio (SNR) to be built up over time using matched filtering. Estimates of the number of important parameters in EMRI evolution range from 7 to 15 . Even taking a number at the lower end of this range, the naive expectation is that $N \sim 10^{35}$ templates would be needed to carry out a fully coherent matched filtering search [116]. This is far more than can reasonably be searched with realistic computing resources. Several alternative approaches to EMRI detection that will be computationally feasible have been investigated. These will be able to detect signals with matched filtering SNR $\gtrsim 20$. By comparison, in a fully coherent search, the SNR required for detection is $12-14$, to ensure a reasonable false alarm rate when searching such a huge number of templates.

LISA data analysis is further complicated by the richness of the LISA data stream. The motion of LISA in its orbit creates amplitude and phase modulation of the signals and we have to employ time-delay interferometry techniques $[14,307]$ in order to remove the laser frequency noise. Time-delay algorithms applied to the Doppler readouts lead to a rather complicated response function which depends on the frequency and sky position of the source under investigation. It is expected that the detection rates for EMRIs will fall somewhere between a few tens and a few thousands [27, 116, 163]. Additionally, the LISA data will be very strongly coloured by gravitational wave signals from the foreground of white dwarf binaries in our galaxy $\left(\sim 10^{7}\right.$ sources which create confusion noise at frequencies below a few millihertz [40, 88, 156, 245]) and signals from a handful of merging supermassive black hole binaries which might have SNR as high as a few thousand [86, 284, 322]. All these signals will overlap in time and frequency. To illustrate this complexity we have simulated a LISA frequency Doppler shift measurement with $\sim 27$ million Galactic binaries, 1 EMRI and 1 inspiralling MBH binaries. The power spectral density of each separate source and the total envelope is presented in figure 4, produced using the methods outlined in [17, 67] and software written by MLDC task force ${ }^{6}$. This figure is primarily illustrative, but it appears that the EMRI signals will be overwhelmed by the signals from the MBH mergers. However, it should be possible to identify and remove the high signal-to-noise ratio $\mathrm{MBH}$ merger signals from the data stream before searching for the EMRI signals, so the situation is not as bad as it may at first appear.

3.1.1. Current status. To date, three algorithms for detection of EMRIs in LISA data have been considered.

The first is a semi-coherent algorithm, which uses a first coherent matched filtering stage to search for $\sim 3$-week sections of EMRIs, followed by a second stage where the power is summed incoherently along trajectories through these sections that correspond to inspirals.

${ }^{6}$ http://sourceforge.net/projects/lisatools. 


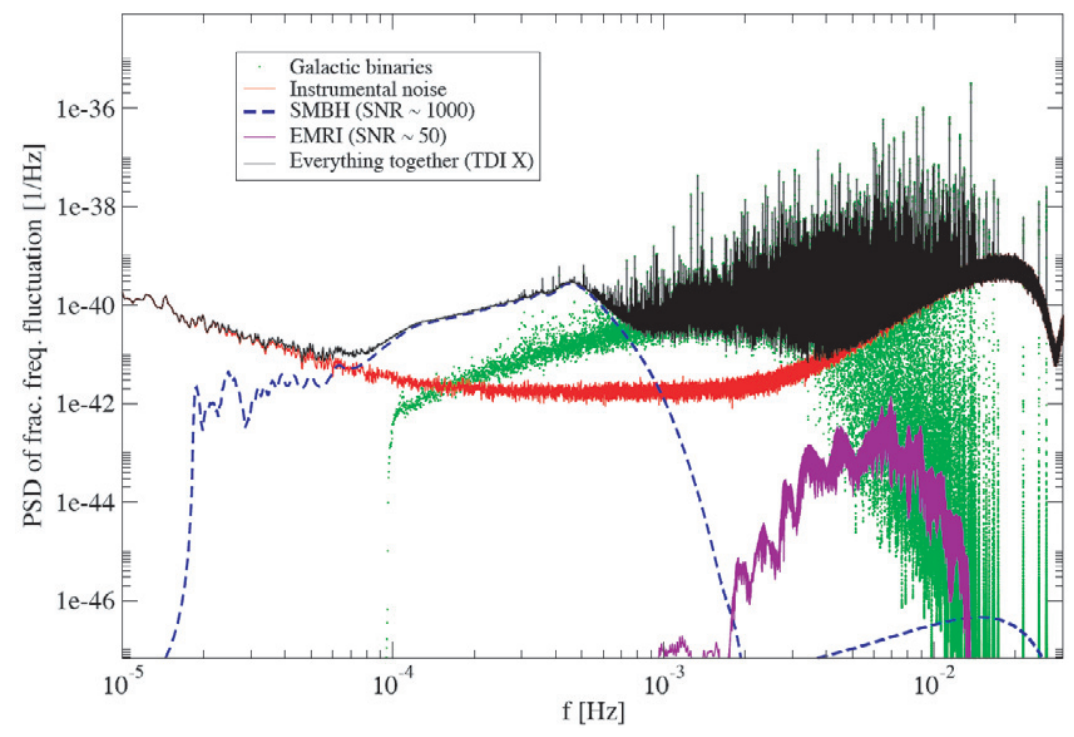

Figure 4. Power spectral density of one of the unequal arm Michelson TDI channel. It contains $1 \mathrm{MBH}$ inspirals at luminosity distances of $3.3 \mathrm{Gpc}$ and 1 EMRI at luminosity distances of $2.3 \mathrm{Gpc}$. The duration of the EMRI was taken to be one and a half years. The galactic binary realization used here was drawn from the distribution described in [245].

This algorithm could detect EMRIs at redshift $z \sim 1$, which translates to tens to hundreds of LISA events, depending on the intrinsic astrophysical rate [116]. The preliminary analysis of this algorithm made only limited efforts to optimize its performance. It is likely that optimization, such as the addition of extra stages in the hierarchy, will be able to further improve the reach of search, but this has not yet been explored.

A second approach is to use time-frequency methods, i.e., divide the data stream into segments of a few weeks in length, perform a Fourier transform on each and then analyse the resulting spectrogram. A simple method that looks for unusually bright pixels in binned versions of this spectrogram could detect typical EMRI signals at about half the distance of the semi-coherent search, but at a tiny fraction of the computational cost [115, 319]. An improved method that considers clustering of bright pixels in the binned spectrograms (the Hierarchical Algorithm for Clusters and Ridges), has slightly further reach, and also more potential for parameter extraction [114]. While more work needs to be done, template-free techniques could detect as many as one tenth of the EMRI sources in the data stream. A typical spectrogram for an EMRI signal is presented in figure 5 for which the amplitude of the signal at plunge was normalized to one.

The third approach that has been explored is to use Markov Chain Monte Carlo (MCMC) techniques. The MCMC approach essentially carries out fully coherent matched filtering, but does so in an intelligent way, reducing the number of waveform templates that have to be considered. MCMC methods are being explored extensively for application to all aspects of LISA data analysis [65, 310, 320]. In the context of the EMRI search, the MCMC approach has been found to work well when searching for a simple model EMRI signal in a short stretch of LISA data [295]. The exact reach of the MCMC search has not yet been properly assessed. Given infinite computing resources, the MCMC would eventually return the posterior probability function for the source parameters. The ability of correctly identifying sources then depends on the shape of this posterior, which depends on both the signal-to-noise 


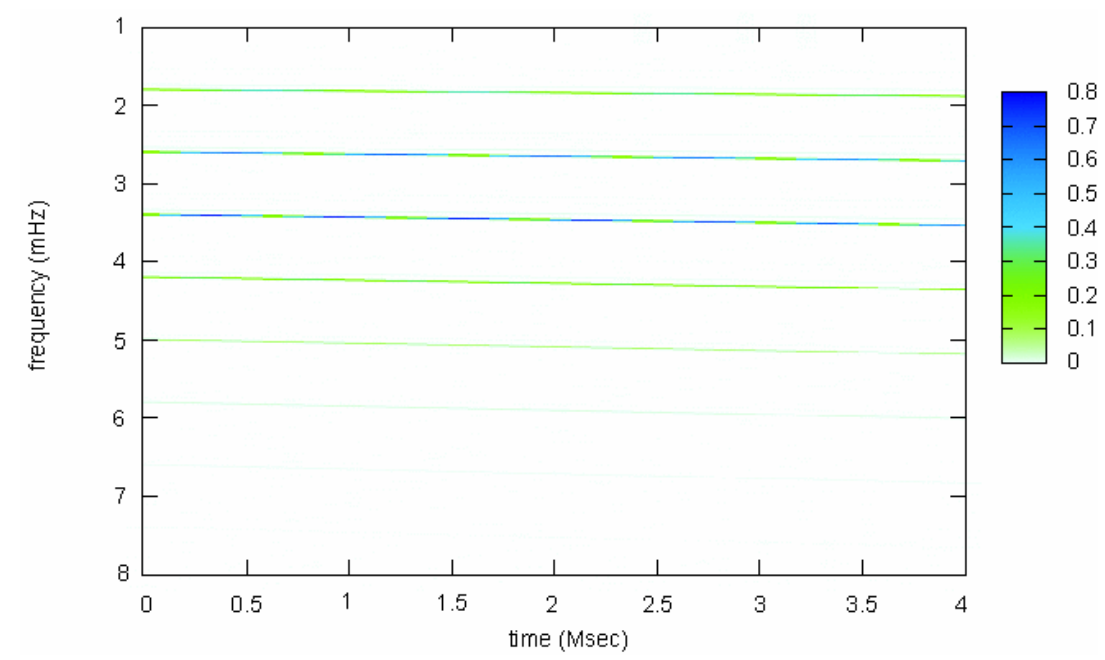

Figure 5. Spectrogram of the signal from an EMRI on an inclined and eccentric orbit. One can see several harmonics modulated by orbital precession and LISA's orbital motion.

ratios of the sources and the structure of the waveform template space. These are the same properties that determine the detection limit of a fully coherent matched filtering search, so it is plausible that an MCMC search with infinite computing power could achieve the same range as the fully coherent matched filtering approach. However, the MCMC suffers from the same computational constraints as the fully coherent search, and these will limit the ability of an MCMC search to sample the posterior. Peaks in the posterior will have to be larger to be detected, and sources will have to be correspondingly closer. For these reasons, it is not possible to say how the performance of the MCMC and the semi-coherent method will compare in practice, although this should become clear over the next several years.

3.1.2. Outstanding challenges. The results quoted above for the various search algorithms were obtained using a vastly simplified model of LISA - searching for a single EMRI event in coloured Gaussian noise. However, as already mentioned, the LISA data stream will actually be source-dominated and in order to detect EMRIs we might first need to clear the data of signals from MBH binaries and from resolvable Galactic binaries. As many as $10^{4}$ Galactic binaries with frequencies above a few $\mathrm{mHz}$ will be individually resolvable [88] and hopefully removed. Estimates suggest there could be tens of coalescing MBH binaries observed each year during the mission lifetime [284]. LISA could also see as many as several hundred individually resolvable EMRI events [116] plus a confusion background generated by distant EMRI signals [27]. In the work on EMRI searches, the confusion background from unresolvable compact binaries has been included in an approximate way, but no account has been made of the effect of interference from the thousands of resolvable sources that will be present in the data. Research on the problem of resolving individual sources in the rather complex LISA data is under way within the Mock LISA Data Challenge effort $[15,16]$.

Some of the questions that need to be addressed are as follows.

- How do the three existing algorithms perform when applied to data streams containing two, ten or a hundred EMRI events? How does the performance degrade when there are other sources, e.g., an MBH merger signal, in the data stream? The semi-coherent and MCMC algorithms are likely to be better at handling confusion than the time-frequency 
approach, since the former methods use matched filtering. Time-frequency analyses will not be readily able to cope with many sources of comparable brightness that intersect in the time-frequency plane. Work needs to be done to quantify these statements.

- If existing algorithms for the extraction of compact binaries and supermassive black hole mergers from the data stream are used on a data stream including one or more EMRI signals, how is the EMRI signal affected? Can the process of 'cleaning' other sources be modelled merely as an alteration in the noise properties of the data stream? This will answer the important question of whether it is necessary to fit simultaneously for all the sources in the data, or whether the parameters of the different source types can be estimated sequentially, before following up with a global fit and refinement of the parameters. Another concern here is how well we can model MBH merger signals- the mismatch between the true signal and the theoretical model could result in rather high residuals.

- Markov Chain Monte Carlo (MCMC) techniques in principle can search for multiple types of source in the data stream, but how well do they perform when searching for compact binaries, supermassive black hole mergers and EMRIs in the same data set? How quickly does the chain converge? How complex is the likelihood function with many different source types in the data stream? It has recently been shown that MCMC based methods can detect $\mathrm{MBH}$ inspirals in the presence of a Galactic WD binary foreground to a very high accuracy [66], which gives reason to hope that this problem will be surmountable.

- What are the computational costs of the various approaches? Which of them are computationally feasible? As mentioned earlier, fully coherent matched filtering is impossible due to the large number of templates required to tile the whole parameter space. The semi-coherent algorithm was designed to make maximum use of expected computational resources, and 3 weeks was estimated to be the longest possible 'snippet' length under that assumption. Markov Chain Monte Carlo techniques provide a more computationally efficient way to search high-dimensional parameter spaces. The MCMC search for a simplified EMRI in 1 month of data described in [295] required the evaluation of $\sim 10^{7}$ chain states in order to determine the parameters of a source which had SNR $\sim 10$. This is fairly typical of the number of states required to accurately recover the posterior in searches for small numbers of sources [66]. However, the exact number of states required will depend on the number of sources in the data (typically a linear scaling), the complexity of the waveform space and the SNRs of the various sources. This will likely increase the requirement by several orders of magnitude, although the MCMC should be able to obtain reasonable estimates of the parameters of the loudest sources using many fewer than the $\sim 10^{35}$ templates required to cover the whole parameter space in a fully coherent matched filtering search [116]. However, the template at each one of these Markov chain states still needs to be evaluated as the chain runs. The templates thus either need to be generated 'on-the-fly' or a bank of templates needs to be generated in advance. For the former, we would need quick methods to generate EMRI waveforms, such as the kludge models. For the latter, we are back to the necessity that the entire parameter space be covered with EMRI templates. This is unlikely to be computationally practical, and even if it were, the overhead associated with accessing such a huge database would be prohibitively high. Thus, computational costs will have to be considered very carefully when devising the final EMRI search.

The final EMRI search will most likely include a combination of the three approaches described above, and perhaps some new techniques. The search is likely to be hierarchical, using inaccurate but quick techniques to get estimates of the source parameters before refining with more computationally intensive methods. It is likely that the searches for different types 
of LISA source will be somewhat integrated with one another, although one possible approach might be to estimate the parameters for each source type separately, before unifying everything in a final global fit.

As described in section 2.1.4, there are various 'non-standard' channels for EMRI formation that may produce comparable numbers of inspirals as the standard picture. A binary tidal separation or the capture of the core of a giant star would tend to lead to an EMRI on a circular orbit. The formation of stars in a disc would tend to lead to an EMRI on a circular and equatorial orbit. These special types of orbit have less free parameters than a generic EMRI. It might therefore be worthwhile having three EMRI data analysis pipelines, focussed on circular-equatorial, circular-inclined and eccentric-inclined orbits respectively. The reduction in the parameter space in the restricted cases will probably not be sufficient to allow fully coherent matched filtering to be carried out, but it will allow considerably longer segments to be used in the first stage of a semi-coherent search. Moreover, the threshold SNR required for detection of a source will be reduced (since there are far fewer templates in which a false alarm could be found), increasing the range of the search. It will be important to find out to what distance a well-tuned search algorithm targeting circular or circular-equatorial EMRIs can make a detection, but this has not yet been explored thoroughly.

No algorithms have been examined explicitly in the context of IMRI detection, although the above algorithms for EMRI detection can all be applied. For the matched filtering algorithms, the complication is the need for waveform models, which will be discussed in more detail below. The time-frequency algorithms can see IMRI events further away, since they are intrinsically brighter, so this might be a good method to use, but it will depend on the distance to the nearest likely event. As search techniques for EMRIs and MBH mergers are further developed in the future, they can be expanded to encompass a search for IMRIs.

\subsection{Source modelling}

Most of the data analysis algorithms outlined above require models of the source waveforms. Waveform templates will also be essential for parameter estimation once sources have been detected. Templates for EMRIs could be constructed in several ways which we discuss here.

\subsubsection{Current status.}

Post-Newtonian expansion. The post-Newtonian expansion in powers of velocity $v / c$ converges poorly when $v / c \gtrsim 0.3$ [45]. Unlike comparable-mass inspirals, which only spend a few cycles in the regime where the post-Newtonian approximation breaks down, IMRIs and EMRIs may spend thousands to millions of cycles in this regime. Therefore, this expansion is not useful for EMRI or IMRI waveform modelling.

Numerical relativity. Solving Einstein's equations numerically on a computer has proven to be a very difficult task, but significant progress has been made in the past year. Several groups have now successfully modelled the last orbit, merger and ring-down of a comparable mass binary system [21, 48, 49, 268]. Numerical techniques are essential for modelling the highly nonlinear dynamics during the last few orbits and merger of a comparable mass system. However, numerical techniques are not fast enough to evolve the large number of cycles necessary for EMRI waveforms. In addition there are technical problems which make numerical methods unreliable as one goes to higher mass ratios. Fortunately, in EMRI systems the extreme mass ratio makes it possible to produce templates accurate over many cycles by 
perturbative methods expanding in the mass ratio, so numerical relativity is not needed in this context.

Self-force waveforms. The extreme mass ratio in an EMRI system allows the waveform to be obtained by perturbation theory. The inspiralling object can be regarded as a small perturbation on the background spacetime of the central black hole, except very close to the small object. In the vicinity of the small object, the spacetime can be regarded as a point mass moving under the influence of an external tidal field due to the central body. Matching these two regimes allows one to obtain an expression for the self-force acting on the small body as a result of its motion in the spacetime. This self-force can be thought of as arising from gravitational radiation being generated by the small object, reflecting off the curvature of the background spacetime and then subsequently acting on the small body. The mathematical theory of this self-force interaction has been developed over the past ten years (see the review [257] and references therein). In principle, the self-force formalism will provide accurate EMRI waveforms that can be used for source characterization. However, evaluation of the self-force is computationally difficult. Recently, a new scheme was proposed [29] which has produced results for the self-force acting on circular orbits in the Schwarzschild spacetime [30]. However, it is computationally expensive to generate the self-force acting even at a single point in an orbit. A generic inspiral trajectory and waveform from a particle evolving as a result of the self-force is still some way in the future.

Adiabatic inspiral waveforms. Evaluation of the self-force acting at every point on the orbit is necessary to include the 'conservative' piece of the self-force, i.e., the piece that modifies the orbit, but does not dissipate energy, $E$, angular momentum, $L_{z}$, or Carter constant, $Q$, (a generalized angular momentum squared which is the third integral of the motion for orbits in the Kerr spacetime). However, the radiative piece of the self-force can be determined more easily, by solving the perturbation equations for the background spacetime, with a source that represents a particle moving on a geodesic of this background. This reduces to integrating the Teukolsky equation [304, 305]. Solutions of the Teukolsky equation encapsulate radiation at infinity in a single equation for the Weyl scalar $\psi_{4}$ :

$$
\psi_{4}=\frac{1}{2}\left(\ddot{h}_{+}-\mathrm{i} \ddot{h}_{\times}\right) \text {. }
$$

The Teukolsky solution also determines the orbital averaged rates of change of the orbital constants- $\langle\mathrm{d} E / \mathrm{d} t\rangle,\left\langle\mathrm{d} L_{z} / \mathrm{d} t\right\rangle,\langle\mathrm{d} Q / \mathrm{d} t\rangle$-from which the value of these constants a short period of time later can be determined. This allows the construction of 'adiabatic waveforms' [170] - a sequence of geodesics can be found that represent an inspiral, by solving the Teukolsky equation for a given geodesic, then computing the energy, angular momentum and Carter constant loss rate for that geodesic, neglecting oscillatory terms which average to zero over the orbital period. These are then used to determine the next geodesic in the sequence. The corresponding gravitational waveforms generated on each geodesic orbit can then be stitched together to give an adiabatic inspiral waveform. This procedure works provided the timescale over which the orbit is changing is long compared to the orbital period, i.e., the evolution is adiabatic. For special classes of orbits-eccentric equatorial and circular inclined - the rate of change of the orbital constants is determined by energy and angular momentum balance. It is possible to extract from $\psi_{4}$ (30) the gravitational waveform near the horizon and near infinity, and hence the amount of energy and angular momentum being carried by the waves down the horizon and out to infinity. Equating the loss of energy and angular momentum of the orbit to the sum of the energy and angular momentum fluxes near the horizon and near infinity determines the orbital evolution. This simplification meant that adiabatic inspirals were determined several years ago for both of these special classes of orbit 
$[130,166]$, although the stitching together of waveforms has only been done for circular inclined orbits [165]. Recently, 'snapshot' waveforms and energy/angular momentum fluxes have also been generated for generic geodesics in the Kerr spacetime [79]. Generic geodesics in Kerr have the third integral of the motion, the Carter constant, in addition to the energy and the polar component of the orbital angular momentum. It was originally thought that evaluation of the self-force would be required to correctly evolve the Carter constant. However, it was recently shown that the evolution of the Carter constant can also be determined from the same Teukolsky coefficients that are needed for computing the energy and angular momentum fluxes [232, 281, 282].

This should allow the construction of generic adiabatic waveform templates in the near future. Adiabatic waveforms are accurate except for the omission of the conservative piece of the self-force. There is some debate in the literature about how important this omission will be $[80,265]$. However, the adiabatic waveforms may be accurate enough over a timescale of a few weeks that they can be used for source detection via the semi-coherent algorithm. They may also perhaps find a role in source characterizations and are somewhat less computationally expensive to generate than full self-force waveforms.

'Kludge' waveforms. The adiabatic waveforms, although accurate, are still computationally intensive to compute. For the purposes of scoping out data analysis algorithms for the detection of EMRIs with LISA, it is necessary to generate waveforms in large numbers, e.g., to count the number of templates needed to cover the whole parameter space with sufficiently high overlap. Perturbative waveforms did not fit this requirement, which led to the development of two families of approximate, 'kludge' waveforms, that capture the main features of true EMRI waveforms but are much quicker and easier to generate.

The first family of kludge waveforms use an 'analytic kludge' (AK). They are based on waveforms representing emission from a particle on a Keplerian orbit, as given by Peters and Mathews [253, 255]. The waveform is augmented by imposing relativistic precession of the orbital periapsis and orbital plane, plus inspiral (in an analogous way to how the adiabatic waveforms include inspiral). The precession and inspiral rates are taken from postNewtonian results. These waveforms are described in [26]. The AK approach is 'analytic' since analytic expressions are known for the Peters and Mathews waveforms. This makes the AK waveforms very quick to evaluate. However, they are not particularly accurate in the latter stages of inspiral, since a Keplerian orbit with precessions is not a good approximation to a true Kerr geodesic close to the central black hole.

The second family of kludge waveforms attempts to address this failing by using a true geodesic orbit for the inspiralling particle. The geodesic equations have to be integrated numerically, so the second family is labelled the 'numerical kludge' (NK). The procedure to compute a numerical kludge waveform has two stages. Firstly, a phase-space inspiral trajectory is constructed, i.e., the sequence of geodesics that an inspiral passes through, by integrating prescriptions for the evolution of the three constants of the motion-energy, angular momentum and Carter constant. An initial prescription for this evolution based on post-Newtonian expansions of the Teukolsky function [131] was found to exhibit pathologies in certain regimes. By imposing consistency corrections and augmenting the evolution with higher order post-Newtonian terms and fits to solutions of the Teukolsky equation, a considerably improved prescription for the inspiral has now been obtained [113]. This current inspiral prescription is accurate until very close to the end of the inspiral for circular orbits. It is less accurate for eccentric orbits, but it should be possible to improve this in the future now that Teukolsky data for generic orbits is available. Once a phase-space trajectory has been obtained, the Kerr geodesic equations can be numerically integrated, with the time-dependent constants 
of the motion inserted. The trajectory of the inspiralling particle through the Kerr background is then obtained. The second stage of the NK construction is to construct a waveform based on this trajectory. This is done by identifying the Boyer-Lindquist coordinates of the particle trajectory with spherical polar coordinates in flat space, and applying a weak field gravitational wave emission formula to the pseudo-flat space trajectory. NK waveforms have been constructed using the standard flat space quadrupole radiation formula, the quadrupoleoctupole formula [38] and the Press formula [266], which is derived for fast-motion but weak field sources. All three prescriptions perform well when compared to more accurate, adiabatic waveforms, but there is little gain from using the Press formula in preference to the quadrupole-octupole expression [18].

AK waveforms are not particularly 'faithful' as EMRI templates, i.e., an AK waveform with a given set of parameters does not have high overlap (noise weighted inner product) with a more accurately computed waveform with the same set of parameters. However, they do capture the main features of EMRI waveforms, which has made them a useful tool for scoping out the semi-coherent algorithm [116] and other studies. They may also be quite 'effectual' templates, i.e., for any real EMRI waveform, there may be an AK waveform with different parameters that has a high overlap with that waveform. The AK family of waveforms may thus play some role in the final analysis of LISA data. The NK waveforms are not only effectual but very faithful, because they are built around true Kerr geodesics. For orbits with periapsis greater than $\sim 5 M$ (in geometrical units, where $M$ is the mass of the central black hole), the NK waveforms have overlaps in excess of $95 \%$ with waveforms computed via solution of the Teukolsky equation. With further improvements (outlined below), the NK waveforms are likely to be very useful tools in LISA data analysis, not only for source detection but also for approximate source identification before subsequent follow-up with more accurate templates. In figure 6 we show a snapshot of NK EMRI waveform. This figure serves as an illustration of the structure of the signal.

3.2.2. Outstanding challenges. None of these approaches to source modelling is as yet fully developed. In the case of the self-force formalism, recent progress has been significant, and the self-force acting on particles in circular orbits in the Schwarzschild spacetime has been computed [30]. However, this is only for a small selection of circular orbits. The work must then be extended to eccentric orbits in the Schwarzschild spacetime, then to circular equatorial orbits in the Kerr spacetime before finally moving onto eccentric-equatorial and ultimately eccentric-inclined orbits in Kerr. Although this is a non-trivial progression, it should be achieved within the next five to ten years. By the time LISA flies, it is likely that codes will exist to compute self-force based waveform templates for arbitrary orbits. However, these are likely to be computationally expensive, which is why it is necessary to pursue the alternative models.

Adiabatic waveforms are at a more advanced stage of completion. All that remains is to compute the evolution of the Carter constant for generic orbits, using the results of [232], and to 'stitch together' waveforms for generic inspiral orbits. There are no technical challenges remaining, although computational cost is an issue. Generic adiabatic inspiral waveforms should be available within one to two years. Understanding their range of validity may take longer, without self-force templates to compare them against. However, the consideration of conservative corrections outlined below will be important for developing this understanding.

The NK waveforms can also be improved. One of the reasons that the performance degrades for small periapsis is that the kludge waveforms do not include tail contributions, i.e., back-scattering of the radiation from the background geometry. It should be possible to include this in an approximate way, which is likely to improve the NK performance for 

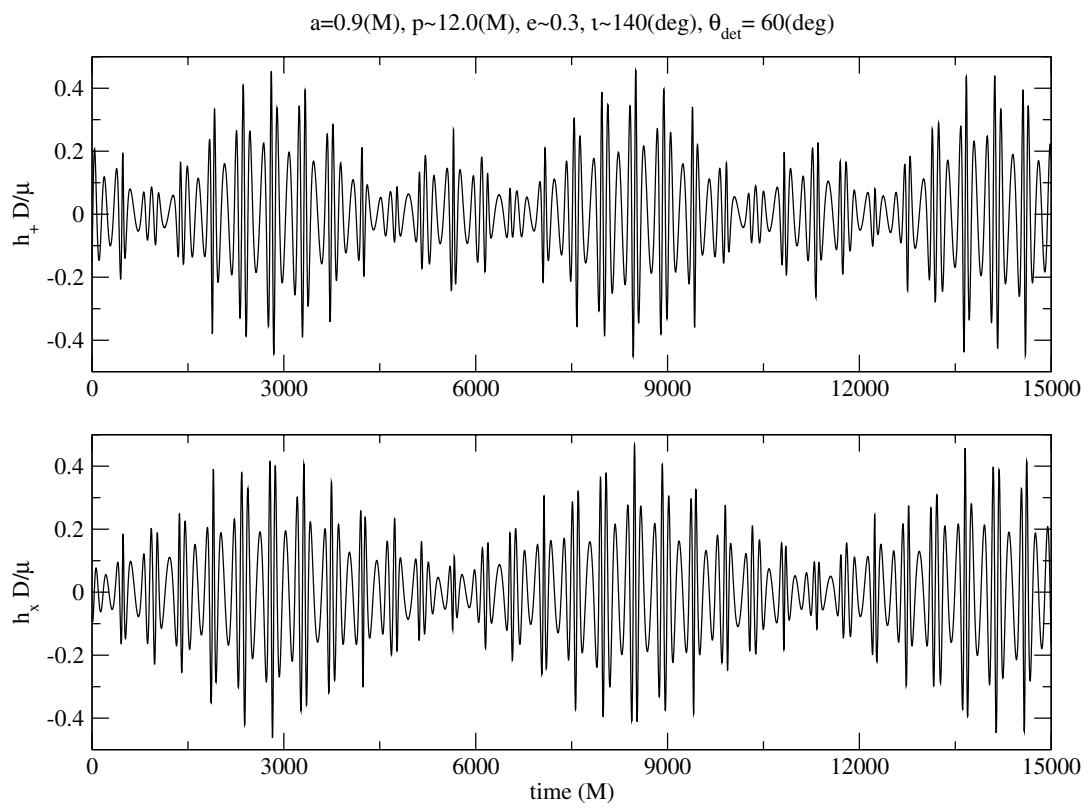

Figure 6. The two polarizations of an NK EMRI waveform with a mass ratio of $10^{-7}$. The GW amplitude is measured in units of the mass of the compact object over distance $(\mu / D)$ and time is measured in units of MBH mass $M$. The eccentricity is $\sim 0.3$, the semi-latus rectum $p \sim 12 M$, the inclination of the plane to the $\mathrm{MBH}$ spin axis is $140^{\circ}$ and the detector (observer) is $30^{\circ}$ above the azimuthal plane.

orbits close to the central black hole. Additionally, the inspiral prescription can be improved by fitting functions to Teukolsky data for generic orbits. The current inspiral prescription [113] includes fits to Teukolsky data for circular inclined orbits, and the resulting inspiral trajectories agree very well for that class of orbit. It is likely that similar accuracy can be obtained for generic orbits in a similar way. Finally, the NK waveforms can be augmented by inclusion of conservative corrections. The conservative correction to the phase evolution of an EMRI is already known in post-Newtonian theory to $3.5 \mathcal{P N}$ order. By considering asymptotic observables, namely the rate of change of the orbital, periapsis precession and orbital plane precession frequencies as functions of those three frequencies, it is in principle possible to compute the necessary conservative corrections for inclusion in the NK model. This was demonstrated for the simple case of circular equatorial orbits in Schwarzschild in [18]. Although the conservative effects could be included this way only up to a certain $\mathcal{P N}$ order, it is quite plausible that this will suffice, since conservative effects contribute most significantly to the phase evolution in the weak field [265], where the $\mathcal{P N}$ expansion is valid.

This last improvement of the NK model is potentially important, since an issue that needs to be understood before LISA flies is how much conservative effects can influence the emitted waveform. If conservative effects are not significant, then a combination of kludge and adiabatic waveforms will be able to identify the parameters of EMRI sources in the LISA data stream with quite high accuracy. If conservative effects are important, the degree to which these waveform families can constrain the parameter space will be significantly reduced. Although self-force calculations are now at the point of computing conservative corrections, this has only been done to date for circular orbits in Schwarzschild. It is unlikely that generic conservative corrections from self-force calculations will be available in the near future. The $\mathcal{P} \mathcal{N}$ fitting 
procedure outlined above will provide approximate results on a much shorter timescale. This should allow the contribution to the phasing of each $\mathcal{P N}$ order in the conservative correction to be assessed, which will give some insight into the importance of including conservative effects. Moreover, the procedure for including the $\mathcal{P N}$ conservative corrections in the NK model can also be used to include these effects in the adiabatic waveforms.

As these developments and improvements to each family of waveforms proceed, we will be able to address three primary groups of questions of great importance.

- What is the computational cost of evaluating waveforms of each type (self-force, adiabatic, kludge)? How many self-force templates could be generated in a reasonable time for a follow-up analysis? How accurately would kludge/adiabatic templates therefore have to constrain the source parameters prior to the self-force analysis?

- What is the overlap of kludge, adiabatic and self-force templates with one another? How accurately, therefore, can kludge and adiabatic templates determine the source parameters, assuming we consider 'faithful' searches only? Can we compute sufficiently many selfforce waveforms to determine parameter mappings between the families, i.e., can we allow the search to be only effectual?

- What is the most computationally efficient way to detect and identify a source, assuming we are using a multi-stage search employing kludge, adiabatic and self-force templates at different stages of this search? What limit on parameter extraction accuracy is set by computational constraints on our search? Is this limit much worse than the theoretically achievable parameter measurement accuracy?

The answers to the first and second itemized points may be incompatible, i.e., we may not be able to determine the parameters of the source sufficiently accurately using kludge or adiabatic templates to allow a self-force follow-up with reasonable computational cost. In this case, we would have to live with a potentially larger error in our source parameters, as mentioned in the third item.

In the above, we have focussed on the modelling of waveforms from EMRIs. However, another outstanding challenge is to develop models for IMRI waveforms. To date, no models have been developed explicitly for IMRIs, although existing models for other systems can be easily applied. An IMRI (mass ratio 1 : 1000) lies somewhere between the inspiral of two comparable mass black holes (a CMRI, mass ratio $\sim 1: 1$ ) and an EMRI system (mass ratio $\sim 1: 10^{6}$ ). For a comparable mass system, the masses and spins of both components are important, but the system spends very few cycles in the regime where the velocity of the components is close to the speed of light. The waveforms can thus be accurately computed from post-Newtonian expansions. In an EMRI system, the number of cycles spent in the highvelocity regime (scaling as one over the mass ratio) is $10^{6}$ times higher, so the post-Newtonian expansion is not reliable, but the extreme mass ratio allows construction of accurate waveforms from perturbation theory. An IMRI is somewhere in between, spending 1000 times longer than a CMRI in the high-velocity regime, but 1000 times less than an EMRI. Post-Newtonian results will not be fully reliable for an IMRI, since they spend so many cycles with $v \sim c$. However, perturbative results are not fully reliable either, since they are linearized in the mass ratio and thus omit terms at higher order in $m / M$ (where $m$ is the mass of the inspiralling object and $M$ is the mass of the central body). In addition, at present we do not have $\mathcal{P N}$ waveforms that include both spins of the two bodies and orbital eccentricity, both of which could be non-negligible for IMRIs.

How quickly do higher order mass-ratio corrections become important? For simplicity, we consider circular equatorial orbits, but the following arguments apply generally. Higher order mass ratio corrections fall into two categories: (i) corrections to the frequency for an 
orbit at fixed radius (these corrections arise from the conservative piece of the self-force and from spin-orbit interactions due to the spin of the smaller body) and (ii) corrections to the inspiral rate. Corrections to the frequency enter at $O(m / M)$, e.g., the spin of the small object is of order $m^{2}$ and leads to a spin-orbit coupling force at this order, with resulting acceleration at order $m$ [250]. However, observationally, we cannot measure the radius of the orbit, only the orbital frequency, so to lay out accurate templates we in fact need the rate of change of orbital frequency as a function of the observable orbital frequency (half the frequency of the fundamental gravitational wave harmonic). Higher order mass-ratio corrections just modify the rate of change of orbital frequency as a function of orbital frequency (as discussed for conservative self-force corrections in [18]). These corrections occur at order $m / M$ above leading order (which itself is $O(m / M)$ since inspiral arises from radiation reaction). A change $\Delta \dot{f}$ in the inspiral rate $\mathrm{d}(\mathrm{Mf}) / \mathrm{d}(\mathrm{t} / \mathrm{M})$ leads to a $\Delta N \sim \Delta \dot{f}(T / M)^{2}$ change in the number of cycles over a dimensionless observation time $T / M$. If the observation time was fixed, this increases like $O\left((m / M)^{2}\right)$, and therefore is much bigger for IMRIs than EMRIs. However, LISA has a fixed frequency bandwidth, and so above a certain mass ratio, inspirals will be observed over a fixed range of frequency. In that limit, the effective observation time is proportional to the number of cycles in a fixed frequency range, $T / M \sim M / m$, which suggests higher order corrections to $\dot{f}$ lead to a phase shift $\Delta N \sim(m / M)^{2}(T / M)^{2} \sim 1$, independent of mass ratio. This argument indicates that perturbative waveform templates will become worse as the mass ratio increases, but at some point the errors will stabilize. Of course, the argument above sweeps many things under the carpet. The orbital frequency is not the only observable, and we have ignored the changes to the GW energy spectrum in the above argument. We have also not considered the size (or post-Newtonian order) of the various corrections to the rate of change of frequency. This argument can be quantified somewhat by using post-Newtonian models. It is possible to compute the overlap of a $\mathcal{P N}$ waveform, linearized in mass ratio, with the full $\mathcal{P N}$ waveform. This calculation will indicate the relative importance of including higher order mass ratio terms in perturbative models, even though the $\mathcal{P N}$ models are not reliable as EMRI templates. Using a leading order $\mathcal{P} \mathcal{N}$ model, describing the last three years of inspiral of two non-spinning bodies, with central $\mathrm{MBH}$ mass of $10^{6} M_{\odot}$, the mismatch between the linearized and full waveforms increases from $0.001 \%$ (when $m=0.5 M_{\odot}$ ) to $0.01 \%\left(m=1.4 M_{\odot}\right)$ to $1 \%\left(m=10 M_{\odot}\right)$ to $15 \%\left(m=100 M_{\odot}\right)$ to $18 \%\left(m=1000 M_{\odot}\right)$. This is consistent with the above argument. For lower mass central black holes, the mismatches are likely to be higher since the plunge frequency is correspondingly higher. There is also likely to be a significant increase in the mismatch when spin is included.

In summary, perturbative templates without higher order mass ratio corrections or spinorbit coupling corrections will probably not be good enough as IMRI templates. However, it might be possible to construct 'Kludge' IMRI templates by including post-Newtonian spin-orbit and other corrections in the current kludge EMRI models (in the same way that conservative corrections are currently being included). This needs further investigation.

\section{Testing relativity theory}

One of the potentially exciting payoffs from EMRI and IMRI observations made by LISA is the ability of testing aspects of relativity theory. The high mass ratio ensures the small object acts like a test particle moving in the background spacetime of the central black hole. The emitted gravitational waves trace out the orbit of the particle, which in turn encodes a map of the spacetime [278]. EMRI events are comparatively 'clean' systems, and we know what this map should look like if the inspiral is an inspiral into a Kerr black hole. Decoding the map then allows us to measure the parameters of the system to high precision. However, 
if the inspiral deviates from what we expect-an inspiral, described by general relativity, of a compact object falling in vacuum into a Kerr black hole-we should be able to see this deviation in the emitted gravitational waves.

\subsection{Current status}

Conceptually, tests of a theory fall into two general categories: comparisons of rival theories (which theory is best supported by the data?) and tests of consistency (are the data consistent with a given theory?).

4.1.1. Comparisons of rival theories. Currently there are no really plausible rivals to general relativity; rival theories have either been ruled out, or, like Brans-Dicke, can be dialled arbitrarily close to general relativity by adjustment of extra parameters (and so could never be ruled out even if GR were completely correct). Moreover, to test an alternative theory, we need to be able to compute gravitational waveforms for EMRIs in that alternative theory to compare against EMRI gravitational waves from relativity. This is a very challenging problem both theoretically and computationally.

Constraining the parameter space that rival theories can occupy can be a useful exercise, since it is a measure of how close to general relativity (GR) the true theory must lie. It is possible to compute the leading-order correction to the gravitational wave phasing for inspiralling objects in Brans-Dicke theory. Work on this has suggested that LISA observations of neutron stars inspiralling into $\sim 10^{2}-10^{4} M_{\odot}$ black holes could put meaningful constraints on the BransDicke parameter and on the mass of the graviton [41, 321]. The theoretical waveforms used for this work were rather simple and ignored important effects such as orbital plane precession due to spin-orbit coupling. It is known that parameter estimation accuracies for MBH binaries improve significantly when spin-orbit coupling is included [186, 314], so the current results are probably conservative. However, it is unclear whether the astrophysical rate is sufficiently high that LISA will be likely to see any of the 'optimal' events for such an analysis $\left(1.4 M_{\odot}+\right.$ $\left.10^{3} M_{\odot}\right)$. Moreover, developing highly accurate waveforms under alternative theories is difficult, and the constraints that I/EMRI observations will be able to impose on the parameter space will probably not be significantly tighter than existing results. For these reasons, most research to date has focussed on the second type of test-tests of consistency.

4.1.2. Tests of consistency within general relativity. A simple example of a consistency test would be to divide an observed EMRI signal into several consecutive pieces, and show that the best-fit parameters from each piece were consistent with each other, within the error bars. This would already be a very strong test of the theory. The complication in regarding EMRI observations as consistency tests is that taking GR to be the correct theory of gravity is not the only assumption that goes into generating the waveform. We assume also that the system is vacuum and that the central body is described by the Kerr metric. Without requiring an alternative theory, we can regard EMRI observations as testing the premise that massive compact objects in our universe are Kerr black holes, rather than some other exotic object (e.g., a boson star or naked singularity) that is still consistent with relativity. Any axisymmetric, vacuum spacetime in relativity can be decomposed into mass $\left(M_{l}\right)$ and current $\left(S_{l}\right)$ multipole moments [120, 145] and it was demonstrated by Ryan [278] that these multipole moments are redundantly encoded in gravitational wave observables, namely the periapsis precession frequency, the orbital plane precession frequency and the gravitational wave energy spectrum for nearly circular, nearly equatorial orbits. If an object is enclosed by a horizon and there are no time-like curves exterior to the horizon, then the object must be a Kerr black hole (this is 
the 'no-hair' theorem), and all of its multipole moments are determined by the mass and spin of the black hole [145]

$$
M_{l}+\mathrm{i} S_{l}=M(\mathrm{i} a)^{l}
$$

where $M$ is a black hole's mass and $a$ is the reduced spin of the black hole $a=S_{1} / M$. If three moments of the spacetime are extracted from the gravitational wave emission, these can be checked for consistency with (31). A boson star with large self-interaction (one viable alternative to an $\mathrm{MBH}$ ) is uniquely characterized by three multipole moments [279], so if four moments are extracted from the gravitational wave emission, the boson star model could also be ruled out. This idea of measuring multipole moments is sometimes referred to as 'testing the no-hair property' (one sometimes hears the variation 'testing the no-hair theorem', but this is obviously sloppy wording, since a true mathematical theorem cannot be invalidated by any experimental test). Objects with non-Kerr values of higher multipole moments within general relativity would have to be exotic stars or naked singularities. The no-hair theorem applies only in relativity, thus non-Kerr values of the higher multipole moments could also arise if the object is a black hole, but relativity is the wrong theory of gravity. If, for example, the quadrupole moment of the massive object was found to differ from that of a Kerr black hole, this could, therefore, have several explanations. We will discuss this in more detail later.

A significant amount of work has been done on quantifying how well LISA could measure multipole moments and carry out these sorts of tests. Ryan [280] considered a general axisymmetric spacetime, decomposed into multipole moments and found that a LISA observation of a nearly circular, nearly equatorial EMRI could measure the mass quadrupole moment to an accuracy of $\Delta M_{2} / M^{3} \sim 0.0015-0.015$, depending on the source parameters. Ryan's approach is somewhat unwieldy, however, since an infinite number of multipole moments are present in the Kerr spacetime. The multipole expansion is essentially an expansion in $1 / r$, where $r$ is the distance from the black hole. LISA will mostly observe EMRIs that are deep in the strong field region, very close to the black hole and in that regime all the multipole moments will be important. Extracting multipole moments one at a time is therefore a rather inefficient way to characterize a Kerr black hole. Collins and Hughes [64] were the first to suggest an alternative approach to LISA observations-regarding the observation as a null-hypothesis test of the assumption that the EMRI is a Kerr EMRI. By constructing spacetimes that are close to Kerr, which Collins and Hughes called 'bumpy black holes', it is possible to quantify how large a deviation from the Kerr spacetime could be present while leaving the signal observationally consistent with a Kerr inspiral [168]. This approach is preferable, since the Kerr spacetime can then be recovered exactly by dialling a small parameter to zero, so we do not lose much sensitivity to the events that we expect to see by using detection templates with an additional bumpy parameter. Collins and Hughes constructed a static spacetime that deviates from the Schwarzschild spacetime by a small amount. They did this by using the Weyl metric and adding a perturbation that represents a pure mass quadrupole asymptotically. They found that the azimuthal frequency of equatorial orbits with the 'same parameters' differed by $0.01 \%$ (for an orbital periapsis of $\sim 50 M$ ) $-10 \%$ (for periapsis of $\sim 6 M$ ) when a quadrupole moment perturbation $Q=0.01 M^{3}$ was added.

Babak and Glampedakis [129] carried out a similar calculation for stationary spacetimes that deviated from Kerr by a small amount, which they constructed using the Hartle-Thorne approach. They not only considered orbital frequencies, but also constructed kludge waveforms and found that in the presence of a $~ 10 \%$ deviation in the quadrupole moment of the spacetime, the overlap could degrade by $25 \%$ over the radiation reaction timescale for typical LISA events. In both these analyses, the results were not directly relevant to observations, since they took no account of the fact that some of the differences in the waveforms could 
be mimicked by changing the orbital parameters (Babak and Glampedakis did comment on this fact in their paper, however). Recently, Barack and Cutler [28] have done an analysis accounting for parameter correlations, using a waveform model constructed by including a term representing a non-Kerr value of the quadrupole moment of the central black hole into their analytic kludge [26]. They find that LISA could measure the quadrupole moment, $Q=-S^{2} / M$, of the central black hole to an accuracy $\Delta Q / M^{3} \sim 10^{-3}$, while simultaneously measuring the mass and spin to an accuracy of $\sim 10^{-4}$. We note that spinning boson stars typically have quadrupole moments ten to a hundred times larger than Kerr black holes of the same mass and spin [279]. This provides an indication of the accuracies required to do meaningful tests.

The research described above concerns extracting information about the spacetime multipole structure from the inspiral part of the waveform. If a ringdown was also detected, the ringdown frequencies can also be used to measure the multipole structure of the ringing object [42]. EMRI-induced ringdowns are unlikely to be detected with sufficient signal-tonoise ratio, but IMRI-induced ringdowns could plausibly be used for such a test. However, more work needs to be done to quantify this, in particular to compute ringdown frequencies for non-Kerr supermassive objects. Moreover, in general the constraints on the multipole moments obtained from the inspiral will be tighter than those obtained from the ringdown due to the large number of wave cycles that can be observed over the inspiral.

There is an analogous source to EMRIs that might be detected by ground-based gravitational wave detectors, namely the inspiral of a stellar mass neutron star or black hole into a $\sim 100 M_{\odot}$ IMBH (a 'LIGO IMRI'). The event rate for such inspirals is somewhat uncertain (see [161, 203, 256] for discussion and further references). However, if they are detected, these sources have the potential to probe the strong-field regime with more modest accuracy than LISA's EMRIs, and would be observed by Advanced LIGO, i.e., a few years before LISA EMRI events are observed. A significant amount of work has gone into studying these sources [46], and this work also applies to LISA EMRI/IMRI events. Some of the applicable results include the generalization of Ryan's results [278] to more generic cases. Ryan considered only nearly circular, nearly equatorial orbits and ignored the effect of tidal coupling. The generalization to eccentric but nearly equatorial orbits is straightforward, although the generalization to arbitrary orbits is difficult $[46,193]$. An extension of Ryan's theorem to tidal coupling tells us that this coupling is also encoded in (and could be extracted from) the gravitational wave observables $[46,194]$. The inspiralling object distorts the horizon surrounding the central object and orbital energy is lost to gravitational radiation flowing into the horizon as well as out to infinity. These interactions with the horizon can be modelled as a tidal interaction, and characterized in terms of energy being lost to the central body through tidal dissipation. In broad terms, the multipole moments of the spacetime can be extracted from observations of the periapsis and orbital plane precession rates, and from these the rate at which energy is being radiated to infinity can be determined. The rate at which energy is being lost from the orbit can also be measured, by observing the change of the orbital frequency with time. The difference between these two energy fluxes gives the flux of energy going into the central body, which is a measure of the strength of the tidal interaction, and tells us about the structure of the central object [46].

In another aspect of this effort to study EMRIs, Gair et al [117] have studied the properties of geodesics in other classes of 'bumpy' spacetimes. This work has considered two types of nearly Kerr spacetimes - exact solutions in the family of Manko and Novikov [204] that deviate from Kerr in the quadrupole and higher moments, and perturbative solutions constructed via solving the Teukolsky equation and then applying the Chrzanowski-Ori procedure to recover the metric [57]. As mentioned before, the geodesics in the Kerr spacetime possess three 
integrals of the motion, because the Kerr spacetime is one of a special class of GR solutions for which the Hamilton-Jacobi equation is separable [51] and $Q$ arises as the separation constant. Spacetimes that deviate from Kerr, even by a small amount, may not be of separable form, and therefore there is no guarantee that the geodesics will possess a full set of integrals. However, it turns out that the majority of geodesics in most of the spacetimes considered in [117] have an approximate third invariant, and the geodesics are tri-periodic to high accuracy: the waveform phase evolution can be decomposed in terms of harmonics of three fundamental frequencies to an accuracy of one part in $10^{7}$ or better. This means that Ryan's theorem can be applied, and deviations from Kerr show up only in the differences in precession and inspiral rates. However, in a small subset of cases, the geodesics show apparently ergodic motion, with no well defined frequency structure. Although this makes the sources difficult to detect, observations of ergodic dynamics would be a clear signature that the spacetime was not simply Kerr. This is unlikely in practice since the ergodic motion only appears for orbits that are very close to the central object, and this is a regime that is probably not accessible in an inspiral that begins with the capture of a star on a highly eccentric orbit some distance from the central object.

\subsection{Outstanding challenges}

There are several questions that still need to be addressed before LISA will be able to carry out tests of general relativity. These include the following.

- What are the imprints of deviations from Kerr on the waveforms generated by EMRIs on generic orbits? As described above, we have a partial answer to this question already. However, the only work so far that includes the effect of radiation reaction on generic orbits is [28], which uses a very simple model. With further research, it should be possible to make general statements about how deviations from Kerr manifest themselves in the EMRI signal, and how the deviations correlate with other parameters. We want to understand how adding greater complexity in the family of deviations from Kerr causes the determination of other parameters to degrade, e.g., whether a Kerr EMRI could also be well described by a 'bumpy' Kerr EMRI with different parameters. In [280], Ryan found that by adding arbitrary multipole moments (up to $M_{10}$ ), the accuracy with which the mass of the large body could be determined in an observation degraded from one part in $10^{6}$ to one part in 3, although most of this degradation was due to the inclusion of moments up to $S_{5}$. This is a known problem in data analysis: introducing more parameters (especially if they are small) causes effective 'noise' in the parameter space. This would also apply if we wanted to use EMRI observations to also test alternative theories of gravity. Testing everything simultaneously would yield very poor constraints, since there would likely be correlations between, for example, a nonzero Brans-Dicke parameter and an anomalous quadrupole moment. Thus, it might be necessary to decide a priori which deviations from relativity we most want to constrain in order to produce useful statements.

- Are there any 'smoking gun' signatures for deviations from Kerr? For instance, ergodicity in the orbits—which 'nearly Kerr' spacetimes admit ergodic orbits, and can a star end up on these orbits in practice? What are the observational signatures?

- Can matter external to the massive object (e.g., an accretion disc, or other stars) perturb the EMRI orbit sufficiently to leave a measurable imprint on the emitted gravitational waves? How would we detect or recognize such a system? Some work has already been done to estimate the effects of an accretion disc [31, 53, 242], which suggests that these effects are unlikely to be measurable unless the accretion disc is very massive. Such massive discs might be found around MBHs accreting at or near their Eddington limit, 
i.e., in active galactic nuclei. As described in section 2.1.4, star formation in the disc of such systems might lead to EMRI events. In a normal nucleus, accretion could happen if a lot of material was recently dumped in the vicinity of the $\mathrm{MBH}$ by, for example, the disruption of a star or a gas cloud. However, such events occur very infrequently [317]. If a very tight binary, consisting of a main sequence star and a compact object, were to inspiral into a $10^{6} M_{\odot} \mathrm{MBH}$, the MS star would be disrupted at a distance of $\sim 2 \times 10^{-6} \mathrm{pc}$ from the MBH. Most of the bound stellar material would be accreted within a few years $[209,309]$ but it would take $100-10^{4}$ years for the compact object to complete its inspiral. Therefore it seems unlikely that the material of the disrupted companion can either perturb the EMRI or create a clear-cut electromagnetic precursor to it. Nonetheless, if there is a small possibility that such a system could in principle be observed, it is important that we know how to detect it and recognize it, not least because an EMRI interacting with a massive accretion disc might lead to an electromagnetic counterpart. An EMRI with a counterpart is a powerful cosmological probe, so it is valuable to maximize our chances of seeing such events.

Finally, we note that if two EMRIs were occurring simultaneously, this would almost certainly leave a measurable imprint on the emitted gravitational waves. However, this is very unlikely as well. A compact binary of mass $m_{\text {bin }}$ tight enough to survive tidal separation down to a distance $R$ of the $\mathrm{MBH}$, would have to have a timescale for (self-)merger by GW emission, $\tau_{\text {merge }}$, smaller than $\tau_{\text {merge }}<\tau_{\text {insp }}\left(\mathcal{M}_{\bullet} / m_{\text {bin }}\right)^{2 / 3}$, where $\tau_{\text {insp }}$ is the timescale for inspiral into the MBH on a circular orbit of radius $R$. Therefore there cannot be any significant number of binaries tight enough to survive until the last $\sim 100$ years of inspiral. If the binary disrupts earlier it is likely that both stars will find themselves on orbits with vastly different inspiral times and when the faster inspiralling one becomes detectable the other will still be too dim. However, the distant EMRI might cause a detectable orbital perturbation on its ex-companion and this has to be assessed.

- How do we interpret deviations from Kerr if they are observed? There could be several explanations - there could be material external to the black hole; alternatively, the massive object could be some exotic star with a non-singular distribution of matter (e.g., a boson star); yet another possibility is that the massive object is a naked singularity, which would disprove the cosmic censorship conjecture, but would not contradict the no-hair theorem. How could we distinguish these possibilities in an observation? Over a long ( $\sim$ year) observation, it should be relatively straightforward to distinguish the effect of material outside the black hole (an 'external' quadrupole perturbation) from a change in structure of the central object (an 'internal' quadrupole perturbation), since the effect would accumulate differently over the course of an inspiral. The existence/location of a horizon might be determined from gravitational wave observations. While an inspiral into a Kerr black hole would undergo a rapid plunge from the innermost stable circular orbit followed by a ring-down, an inspiral into a boson star may continue to produce inspirallike waves after the compact object crosses the stellar surface. The signal-to-noise ratio generated during the plunge and ringdown for an EMRI will be small, so this will almost certainly not be observed. However, if a signal persisted after the 'plunge', this might be detected by building up the signal-to-noise ratio over several waveform cycles. Thus, we might be able to say whether a signal is still 'present' or 'absent', although the resolution of the time at which the signal became absent would not be very high. A horizon could be inferred by comparing the approximate time of plunge with the plunge time predicted based on parameters measured in the early part of the inspiral. If a horizon is found to be absent, the object might be an extended mass distribution (e.g., a boson star) or a naked singularity. If emission after the object's path started to intersect the boson star material 
was observed with sufficiently high signal-to-noise ratio, the features of this emission might allow us to distinguish between these two possibilities [177]. Only if the massive object were found to have a non-Kerr quadrupole moment that was not due to the presence of exterior matter and a horizon would there be firm evidence that the system did not have the no-hair property; however, proving that any horizon completely surrounds the body and that no closed time-like curves exist in the exterior may be impractical. While these ideas give us some hope that interpretation will be possible, further research is needed on all of these topics.

- How do we detect deviations from GR in practice? The need to use matched filtering for EMRI detection makes it difficult to detect signals that look very different from our template models. It also makes it hard to detect small deviations in the model. The simplest thing we can do is to look only for inspirals into Kerr black holes. If our observations are consistent with this model, then we have tested the theory to high precision. The existing research programme then allows us to make statements such as 'this observation is consistent with a Kerr inspiral, with agreement in the quadrupole moment to $x \%$ '. To do this in practice we would perform Monte Carlo simulations to find the maximum ' $x$ ' such that the gravitational waveform emitted during an inspiral into a non-Kerr object with a quadrupole moment that differed by $x$ from the Kerr value still had sufficiently high overlap (for a suitable definition of 'high') with a waveform emitted during an inspiral into a Kerr black hole with some (not necessarily the same) parameters. A more sophisticated analysis could look at segments of the inspiral separately, and check for consistency in the parameters estimated for each segment. We could also look for characteristic signatures of a deviation from Kerr, for instance a transition from regular to ergodic motion in the orbits or the existence/location of the horizon inferred by the plunge time. This might be done by dividing the end of the inspiral into short segments, in which the signal should have SNR large enough so that we can say with high confidence whether the signal is present or absent. The resolution of the plunge time is thus likely to be poor. If the EMRI is close enough to be loud (SNR $\gtrsim 50$ ), it might be possible to detect the signal in a time-frequency analysis. This would not only make it easier to measure things like the plunge point, but would allow us to detect signals that deviate significantly from Kerr inspirals. Finally, it might be possible to do a more generic analysis using templates parametrized by spacetime multipole moments, e.g., the family employed by Ryan [280]. Such a technique would not be particularly sensitive to Kerr inspirals, but if it was used in conjunction with a matched filtering search for Kerr EMRIs it might be a useful diagnostic. As EMRI data analysis techniques are developed, techniques for spacetime mapping will need to be properly explored.

\section{EMRI science}

It is clear from the discussion of EMRI detection above that, while much is already known, there is still some work to be done before LISA flies. However, the scientific payoffs if we detect and characterize a large number of EMRI events could be very significant. From a single EMRI observation, we can measure the parameters of the system to very high precision [26]. The mass and spin of the central black hole, the mass of the inspiralling object, and the orbit's eccentricity (at some fiducial instant) can all be determined to a part in $10^{4}$, typically, while the cosine of the orbit's inclination angle (roughly, the angle between the MBH's spin vector and the orbital angular momentum of the CO) can typically be determined to $\sim 10^{-3}-10^{-2}$. The luminosity distance to the source can be measured to an accuracy of $\sim 5 \%$, and the sky position to a resolution of $10^{-3} \mathrm{sr}$ (a few square degrees). The accuracies achievable with 
Table 1. This table shows the signal-to-noise ratio (SNR) at a distance of $1 \mathrm{Gpc}$ for systems with a variety of observed masses $M$ and $m$. Also shown is the maximum redshift at which such a source could be detected, $z_{\max }$, and the intrinsic masses of the system, $M_{i}=M /\left(1+z_{\max }\right)$ and $m_{i}=m /\left(1+z_{\max }\right)$, that a source at redshift $z_{\max }$ would need to have in order to have apparent redshifted masses $M$ and $m$. The SNRs were computed assuming the optimal TDI combination of LISA data streams could be constructed for five years of observation. All sources have MBH spin of $S / M^{2}=0.8$, inclination of $45^{\circ}$ and eccentricity at plunge of 0.25 . The waveforms were computed using the numerical kludge model [18, 113] and the LISA response was included using the Synthetic LISA simulator [311]. These results were used for computing event rate estimates using the semi-coherent search [116].

\begin{tabular}{lcclll}
\hline $\mathrm{M} / M_{\odot}$ & $\mathrm{m} / M_{\odot}$ & $\mathrm{SNR}$ at $1 \mathrm{Gpc}$ & $z_{\max }$ & $M_{i} / M_{\odot}\left(z_{\max }\right)$ & $m_{i} / M_{\odot}\left(z_{\max }\right)$ \\
\hline $3 \times 10^{5}$ & 0.6 & 18 & 0.13 & $2.7 \times 10^{5}$ & 0.53 \\
& 10 & 73 & 0.44 & $2.1 \times 10^{5}$ & 6.9 \\
& 100 & 620 & 2.5 & $8.5 \times 10^{4}$ & 29 \\
$1 \times 10^{6}$ & 0.6 & 30 & 0.21 & $8.3 \times 10^{5}$ & 0.50 \\
& 10 & 210 & 1.0 & $4.9 \times 10^{5}$ & 4.9 \\
& 100 & 920 & 3.5 & $2.2 \times 10^{5}$ & 22 \\
$3 \times 10^{6}$ & 0.6 & 25 & 0.17 & $2.6 \times 10^{6}$ & 0.51 \\
& 10 & 270 & 1.3 & $1.3 \times 10^{6}$ & 4.4 \\
& 100 & 1500 & 5.2 & $4.8 \times 10^{5}$ & 16 \\
\hline
\end{tabular}

LISA IMRI observations should be significantly better. In addition, for each of these observed systems we will be able to test the black hole hypothesis to high accuracy (e.g., constrain the mass quadrupole moment to a fraction of a percent), as described in section 4.

LISA may detect as many as several hundred EMRIs out to a redshift of $z \sim 1-2$ $[25,116]$. The first estimates of signal-to-noise ratios for EMRIs were done by Finn and Thorne [96]. They considered circular equatorial inspirals only, and found that at a distance of $1 \mathrm{Gpc}$, one year before plunge, the inspiral of a $10 M_{\odot}$ object into a rapidly spinning $10^{6} M_{\odot}$ black hole would have signal-to-noise ratio of $\sim 100$ in a gravitational wave frequency bandwidth equal to the frequency. To obtain EMRI rate estimates, updated signal-to-noise ratios were computed for inclined and eccentric orbits, using Kludged waveforms [18, 113] and including a more accurate model of the LISA response provided by the Synthetic LISA simulator [311]. Table 1 shows the results of those calculations - the signal-to-noise ratio of a variety of systems at a fiducial distance of $1 \mathrm{Gpc}[25,116]$. These signal-to-noise ratios assume that the LISA mission lasts five years and that the satellite is fully functional for the whole time, so that the optimal combination of TDI data streams can be used. These results are consistent with Finn and Thorne [96] when one accounts for the gravitational wave bandwidth remaining one year from plunge, the fact that Finn and Thorne compute the SNR in one LISA Michelson channel only and the fact that these orbits are eccentric.

To translate these SNRs into a maximum detectable distance, we first need to specify a detection threshold. The estimated detection threshold for the semi-coherent algorithm described earlier is $\sim 30$, although optimization of this method may be able to reduce this threshold somewhat. A GW source at a redshift $z$ with masses $M$ and $m$ looks like the same type of gravitational wave source at a Euclidean distance equal to the luminosity distance to redshift $z, D_{L}(z)$, but with redshifted masses $(1+z) M$ and $(1+z) m$. Table 1 also shows the redshift at which the source would have SNR of 30 (computed by setting SNR $(1 \mathrm{Gpc}) / 30=$ $D_{L}(z) / 1 \mathrm{Gpc}$ ) and the intrinsic masses $M_{i}$ and $m_{i}$ that a source at that redshift would have to have in order to give the appropriate observed masses. This table serves to illustrate the typical distances to which sources can be detected. 


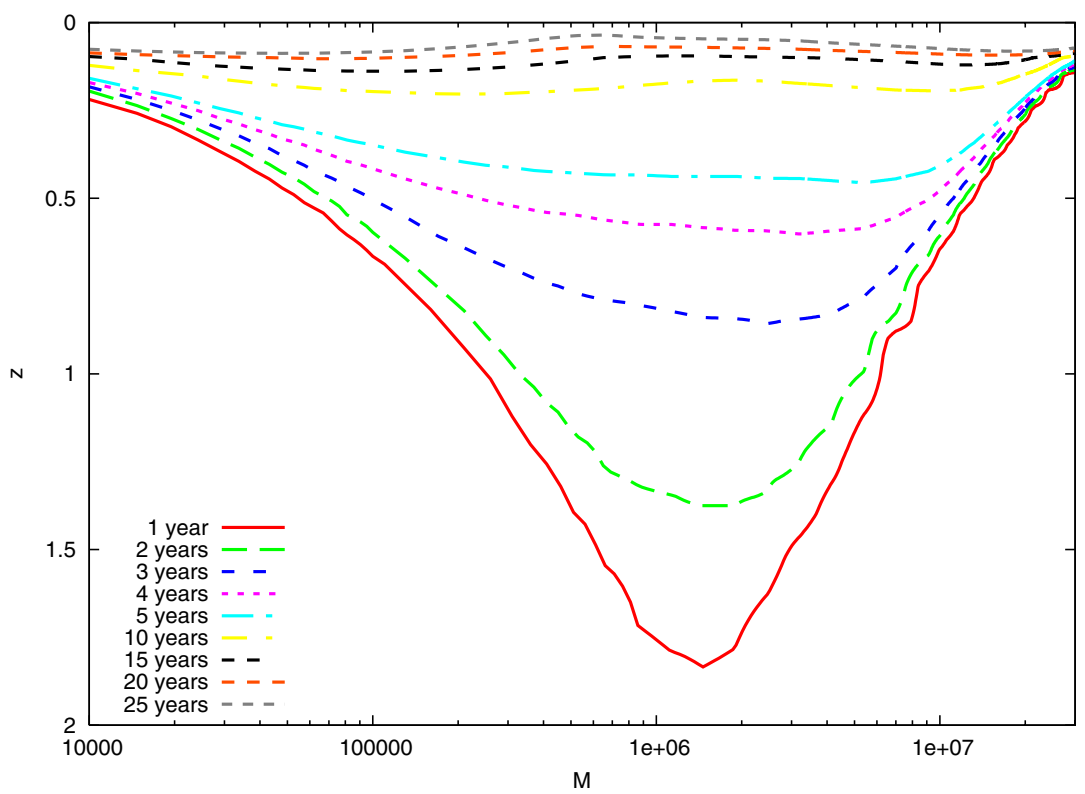

Figure 7. Contours of constant 'detectable lifetime' (as defined in the text) for the circularequatorial inspiral of a $10 M_{\odot}$ black hole into an $\mathrm{MBH}$ with spin $a=0.99$, as a function of $\mathrm{MBH}$ mass $M$ and redshift $z$.

The mass redshifting makes the process of computing the range for a given source a more complicated procedure than a simple linear distance scaling. Figures 7 and 8 show preliminary results of a more careful calculation [112]. The plots show contours of constant 'detectable lifetime' for circular-equatorial EMRI sources as a function of the mass of the central black hole. The figures plot the intrinsic mass of the $\mathrm{MBH}$ on the $\mathrm{x}$-axis, and redshift on the $y$-axis. The lines on the plot are contours of equal observable lifetime, $\tau$. A source will be detectable only if the signal-to-noise ratio accumulated over the LISA mission exceeds the necessary threshold (assumed to be 30 for this plot). This requirement will only be satisfied if the source is in a certain range of phases of the inspiral at the moment that LISA starts taking data. The observable lifetime is the length of that acceptable range of phases, as measured at the source. If EMRIs of this type start once every $T$ years in any galaxy, the expected observed number of events would be $\tau / T$ per galaxy. For figure 7 , we consider only prograde inspirals into a central black hole with spin $a=0.99$ and plot contours for various values of $\tau$. In figure 8 we show $\tau=1$ yr contours for various black hole spins (NB a negative spin indicates a retrograde inspiral into a black hole with spin of the same magnitude). In both plots we have assumed a constant mass for the compact object of $10 M_{\odot}$, and are considering circular-equatorial inspirals only. These results were computed using the flux data tabulated in Finn and Thorne [96], assuming a five-year LISA observation that uses both Michelson channels, averaging over the sky position and orientation of the source, and taking the LISA noise spectral density as given in [26], with the assumption that the white-dwarf background has been subtracted using five years of LISA data. It is clear from this figure that we can see EMRI events out to fairly large distances, but that this distance is very spin dependent. There is also a significant spin dependence of the mass to which LISA has maximal reach. The fraction of the total energy radiated that is radiated in a circular-equatorial inspiral between 


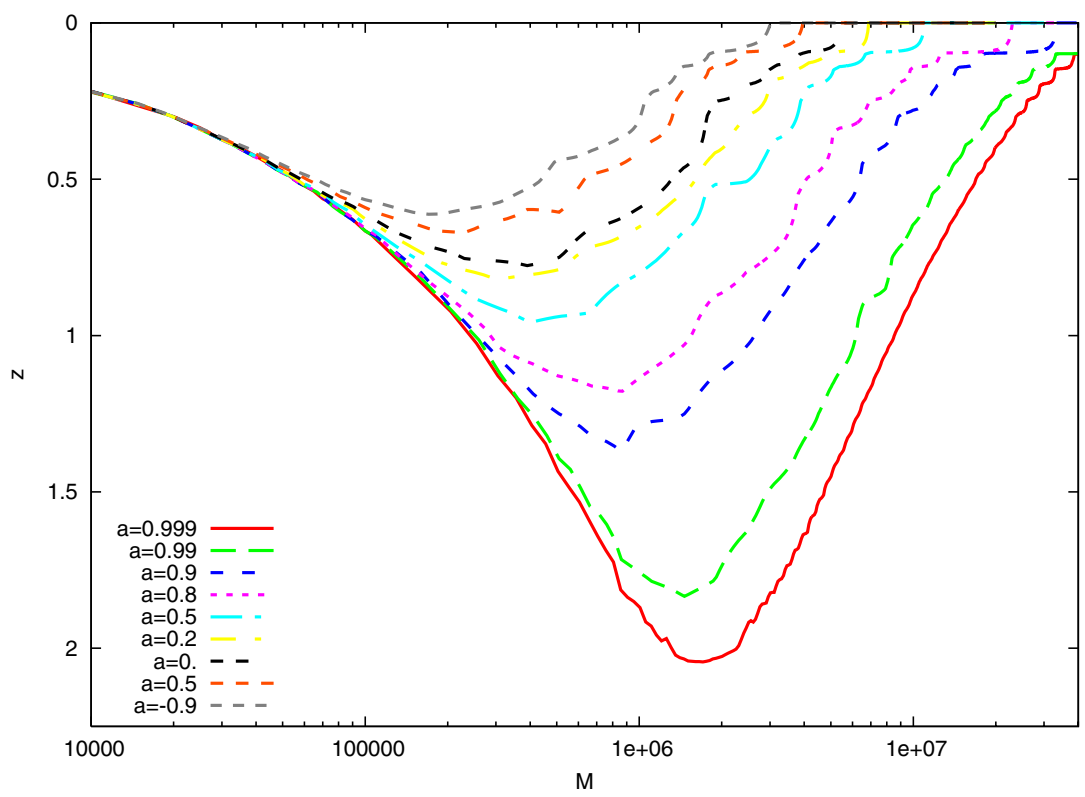

Figure 8. As for figure 7, this figure shows contours of constant 'detectable lifetime', $\tau$, for the circular-equatorial inspiral of a $10 M_{\odot}$ black hole into an $\mathrm{MBH}$. Here we show $\tau=1$ year contours for several different spins of the central black hole, as labelled in the key. Negative spins indicate retrograde circular-equatorial inspirals into black holes of the same spin magnitude.

a Boyer-Lindquist radius $r$ and plunge at the innermost stable circular orbit, $r_{\text {isco }}$, effectively depends on spin only through the ratio $r / r_{\text {isco }}$. The ISCO radius decreases as the black hole spin increases, and the total energy radiated increases. This means that inspirals into rapidly spinning black holes radiate more energy, having, therefore, higher total signal-to-noise ratios, and this radiation is emitted at higher frequencies for a given central black hole mass. LISA will be most sensitive to systems that radiate most of their energy in the detector's optimal sensitivity range of $\sim 3-10 \mathrm{mHz}$. The frequency of the radiation decreases as the black hole mass increases, but increases as the spin increases. The mass that ensures radiation at $\sim 5 \mathrm{mHz}$ will, therefore, be larger for higher black hole spins, as illustrated in the figure. It is also clear from figure 8 that the sensitivity to low mass central black hole systems is effectively independent of spin. For these systems, LISA will observe the middle part of the inspiral, while the final plunge occurs at higher frequencies that are out of band. LISA will, therefore, observe a phase of the inspiral where the radius is large, at which distances the orbit does not 'feel' the effect of the black hole spin.

These results illustrate the LISA range to typical EMRIs, but are only for circularequatorial orbits, and assume a simplified model of the LISA response. Eccentric orbits in general will lose more energy in the LISA band, and hence should have larger signal-to-noise ratio. Treating the LISA data stream more carefully, using a TDI analysis (e.g., by using Synthetic LISA to include the response function [311]), will also tend to increase the signalto-noise ratio, especially for sources that generate significant radiation at high frequencies. These general expectations are supported by the results in table 1 and by further calculations using numerical kludge waveforms $[18,113]$ for eccentric-inclined orbits.

For all of the systems that LISA can see, we will obtain accurate parameter estimates. LISA will thus provide data on a large sample of black holes in the relevant mass and redshift 
range, which can be used for astrophysics. For the discussion sessions at the meeting that inspired this review, we divided the scientific questions on the astrophysical benefits and consequences of LISA EMRI and IMRI observations into five categories. We summarize these in the following sections, along with an outline of the answers that LISA might give us.

\subsection{What can we learn from the characterizations of EMRI/IMRI dynamics, i.e., the observed eccentricities etc of the orbits?}

The observed eccentricities will carry information about the capture mechanism: significant eccentricities are indicative of the direct capture scenario via two-body relaxation, while negligible eccentricities suggest capture via binary tidal disruption or, possibly, tidal stripping of giants (such captures occur at a higher periapsis and have time to circularize before entering the LISA band). The orbital inclination is also informative: random inclinations are expected in the standard scenario with a spherical cluster, while formation in the disc would be manifested by a significant fraction of EMRIs having near-zero inclinations (although this could be made more complicated by disc warpage). The event rates themselves would be interesting, although it may be very difficult to deconvolve the various uncertain parameters that influence event rates (e.g., the $\mathrm{MBH}$ density and mass function, the density and distribution of compact objects near the $\mathrm{MBH}$, etc).

\subsection{What can we learn about the inspiralling compact objects from EMRIs/IMRIs?}

We will learn the $\mathrm{CO}$ mass to fractional accuracy $\sim 10^{-4}$. From the distribution of masses we will obtain information about the relative numbers of the $\mathrm{WD}, \mathrm{NS}$, and $\mathrm{BH}$ populations close to the $\mathrm{MBH}$ (within $\sim 0.01 \mathrm{pc}$ ). Although it is difficult to deconvolve mass segregation from the initial mass function or from the mass dependence in capture rates, unexpected results such as the under-representation of more massive COs could be very intriguing. If BHs with masses in the range $20-1000 M_{\odot}$ are found, this is already an important discovery, since such objects are not yet firmly known to exist (and, if $1000 M_{\odot}$ IMBHs do exist, it is not clear how well they can sink to the centre). Even the precise measurement of the mass of a few stellar BHs (in the range $3-20 M_{\odot}$ ) would be of great interest to constrain models of stellar evolution and collapse. CO spins will probably not be measurable for EMRIs, but we guess they might be measurable to $\lesssim 10 \%$ for IMRIs. This would tell us about the formation mechanism for IMRIs (has mass been accumulated mostly via accretion, or via mergers?), just as for the MBH spin.

\subsection{What can we learn about the MBHs from EMRIs/IMRIs?}

From EMRIs/IMRIs, we will learn the masses and spins of MBHs to fractional accuracy $\sim 10^{-4}$. Rapid spins will imply that much of the MBH mass was built up by gas accretion from a disc (unless the gas arrives in randomly oriented events [179, 180, 274]), moderate spins will imply the $\mathrm{MBH}$ was built as a result of a major merger of comparable-mass black holes [169, 220], while low spins will imply the MBH was mostly built from a sequence of minor mergers with smaller objects coming in from random directions (since then the spin angular momentum increases only in a random-walk fashion). The boundaries that separate these three spin regimes are somewhat uncertain. Spins $S / M^{2}>0.9$ are undoubtedly 'high', but lower spins might also arise from accretion. The spins resulting from major mergers depend on the magnitude and direction of the spins of the two components prior to merger and could be anywhere in the range $0.4 \lesssim S / M^{2} \lesssim 0.9$. Spins $S / M^{2}<0.2$ are undoubtedly 'low', but the boundary between this regime and the major merger regime is unknown. Once 
again, decoding the spin observations must be done carefully. The prograde inspiral of a black hole into a rapidly spinning $\mathrm{MBH}$ has much greater SNR at a given distance than a retrograde inspiral or an inspiral into an $\mathrm{MBH}$ of lower spin. Our observations will therefore be weighted towards more rapidly spinning black holes. Since we can compute the relative SNR of systems with different spins, it will not be too difficult to account for bias when interpreting the observations, but this must be done carefully.

\subsection{What can we learn about cosmology and early structure formation from EMRIs/IMRIs?}

An EMRI or IMRI observation will give a very accurate measure of the luminosity distance to a source, but not an independent value for the redshift at which the source is located. However, it is possible that an electromagnetic counterpart to an EMRI event will be observed (the sky position accuracy of a few square degrees, although poor, is not beyond the reach of survey telescopes). The EMRI will be observed for sufficiently long before plunge that the plunge time can be accurately estimated, and advance warning of the moment of coalescence supplied to other telescopes. However, it is not entirely clear what mechanisms could give rise to an electromagnetic signature at coalescence that would be sufficiently bright to be seen at cosmological distances. If we were lucky, and a single EMRI event was observed with an electromagnetic counterpart, it would provide an estimate of the Hubble constant that is not tied to the local distance scale. We could thus measure the Hubble constant to $\sim 2 \%$, compared to the current systematic error from the Hubble Key Project of $\sim 10 \%$. With $N$ observations, the error decreases like $1 / \sqrt{N}$.

The inspiral of a $10 M_{\odot}$ object into a $10^{5}-10^{6} M_{\odot} \mathrm{MBH}$ should be detectable to a redshift of about 2, corresponding to a time when the Universe was less than 4 Gyr old. Although $\mathrm{MBH}-\mathrm{MBH}$ mergers are visible to much larger redshift, offering the potential to probe the whole early history of $\mathrm{MBH}$ formation and growth, there are considerable uncertainties about their predicted detection rate [142, 208, 284, 322]. Also the merger rate for LISA-detectable systems probably peaks at $z>2$. EMRIs therefore offer an alternative and complementary way to probe the relatively late evolution of MBHs with masses below a few $10^{6} M_{\odot}$.

\subsection{How can EMRIs/IMRIs be used to test GR, or (assuming GR is correct) that the central massive object is a Kerr BH?}

As discussed above, what we can perform are consistency checks (i.e., is the signal consistent with GR predictions?) or compare the Kerr null hypothesis to straw man alternatives. In the simplest case, if we find that the observed EMRI waveforms agree with the models predicted by GR, for reasonable physical parameters, this would be an impressive verification of the theory. Matching strong-field EMRI waveforms to one cycle in $10^{5}$ will be compelling evidence in support of GR. Beyond this, one can further check consistency in the EMRI by checking, for example, that different pieces of the waveform yield the same consistent estimates for the physical parameters of the system. This amounts to verifying the consistency of relativity in the strong field region in the immediate vicinity of MBHs, a regime in which it has not been verified to date (though one hopes this regime will be probed by LIGO before LISA flies, albeit with less accuracy). These consistency checks are not testing the theory against an alternative; however, this is familiar from other areas of physics. For instance, in particle physics, tests of the standard model are not based on comparison with serious rivals: one measures that the $W$ mass or top quark mass is roughly where it is predicted, and this is treated as a substantial validation of the theory. Also, the idea of embedding GR/Kerr predictions in a somewhat larger, phenomenological theory (e.g., with nonzero graviton mass, 
or non-standard quadrupole moment for the BH), is similar to the current status of GR tests with binary pulsars, in which the orbital motion is first fit to a phenomenological set of Keplerian and post-Keplerian parameters, and radio astronomers then show that the fitted values are consistent with the predictions of GR (twenty years ago binary pulsar measurements killed a host of alternatives to GR, but now that they are dead, radio astronomers are also stuck with demonstrating consistency with GR).

One important issue is whether, if systems that differ from the Kerr hypothesis exist, we will actually be able to detect them, given that our data analysis will rely on matched filtering. As mentioned above, if the deviations are small, this will not be a problem, since the source will remain consistent with our templates for long enough to be detected. The point at which deviations start to appear will then be a probe of the nature of the deviations present (e.g., the existence/location of a horizon as determined by the frequency of plunge, if any). If the deviations are large, then matched filtering might fail entirely, and we would have to rely on a source being close enough to show up in a time-frequency analysis or other template-free technique.

If we see a single event that differs from the Kerr model, this will be very weak evidence against the black hole hypothesis (no doubt many possible explanations would appear in the literature over time). If every observation differed, then the evidence would be difficult to refute. Explaining the observations would be a difficult, but extremely interesting task.

\section{Conclusions}

Black hole binaries with large mass ratios are uniquely important sources for planned spacebased gravitational wave detectors such as LISA. These EMRIs or IMRIs will provide information about the stellar dynamics of galactic nuclei that will be difficult, if not impossible, to obtain any other way. Single events will yield precise measurements of the masses and spins of supermassive black holes in a mass range extremely difficult to observe electromagnetically. In addition, the high mass ratios mean that inspiral events will map the spacetime around black holes and test predictions of general relativity in the strong field. These enticing prospects have led to a recent surge in interest in the astrophysical, general relativistic, and data analysis aspects of EMRIs and IMRIs, and we have given here an overview the state of the art in these three areas. Although much analytical and numerical work remains, the level of progress in the last few years suggests that when LISA flies in roughly a decade, the community will be ready to maximize the scientific return from observations of these events.

Currently, the most discussed mechanism for the production of EMRIs involves the gradual evolution of the orbits of stellar-mass black holes and other compact objects via twobody relaxation. The estimated rates for a galaxy such as the Milky Way are in the range of $\sim 10^{-8}-10^{-6} \mathrm{yr}^{-1}$. The further technical development of $N$-body codes will be essential to reducing the uncertainty of these rates, as well as to proper inclusion of effects such as mass segregation and resonant relaxation. There are additional qualitatively different mechanisms that have been proposed recently, including tidal separation of binary stellar-mass black holes, and formation or capture of black holes in accretion discs around the $\mathrm{MBH}$, that could lead to an increase in the estimated EMRI rate. In addition, whereas standard EMRIs are likely to have high eccentricity and random inclination in the LISA frequency range, tidal separations would lead to circularized orbits with random inclination, and disc processes to circularized orbits in the spin plane of the MBH. The distinct waveforms from these different mechanisms suggest that they will be distinguishable in data, and hence will carry important information about different properties in galactic nuclei. 
Detection of EMRI and IMRI signals in the LISA data stream is a difficult task. In section 3.1 we described three existing algorithms for EMRI detection and discussed some of the outstanding issues in EMRI data analysis. The current algorithms might be able to detect as many as several hundred EMRIs in the LISA data stream-the reach of the best searches (semi-coherent and Markov Chain Monte Carlo) is out to $z \sim 1-2$. However, the performance of each of these algorithms has so far been analysed only for the detection of a single EMRI in noisy data. The LISA data stream will be source-dominated, and the need to simultaneously identify and extract all these signals puts severe demands on data analysis algorithms. Understanding how to extract EMRIs in the presence of source confusion is the key data analysis issue that must be addressed in the future.

Several of the proposed data analysis algorithms employ some variant of matched filtering, for which models of the signals present in the data must be known. The extreme mass ratio means that accurate EMRI waveform templates can be constructed using black hole perturbation theory. However, this is computationally intensive. Various approximate EMRI waveform models have been developed, and these were described in section 3.2. Comparison to perturbative results suggests that these models might be good enough for LISA data analysis, but more work needs to be done. A key uncertainty is in the computational costs. A plausible data analysis strategy would use an approximate waveform model to get estimates for the source parameters, before carrying out a follow up search with more accurate waveforms. This hierarchical approach is subject to constraints on computing power. It may turn out that it is not possible to constrain the source parameters with sufficient accuracy in the first stage of the search to perform the follow-up search in a reasonable time. If that is the case, we will end up with larger errors on observed source parameters, but this needs to be quantified as waveform models are further developed in the future. Modelling of IMRI waveforms has not yet been considered in detail. While EMRI or comparable mass binary models or a combination of the two might be applied, this needs further investigation.

One of the key science goals of EMRI observations is to test general relativity theory in the strong field. The extreme mass ratio means that the inspiralling object effectively acts like a test body in the spacetime of the central object. The emitted gravitational waves encode a map of the spacetime of the central object. If we can decode that map, we will be able to tell to high precision if the central body is indeed a Kerr black hole, or some other object. Understanding how LISA will be able to test relativity in practice is a subject of much current research, which we summarized in section 4 . The main outstanding issues include producing waveforms for generic inspirals in bumpy (modified Kerr) spacetimes to test detection and data analysis strategies; finding efficient ways to search for deviations from a Kerr black hole; and interpreting such deviations to determine their origin (e.g., an accretion disc around a Kerr black hole, a boson star, a naked singularity with non-Kerr higher order multipole moments).

If we do manage to detect many EMRI and IMRI events with LISA, we stand to learn a lot about astrophysics, and we summaries some of this discovery space in section 5 . The EMRI eccentricity distribution can tell us about the capture mechanisms, while the distribution of inclinations can shed light on whether compact objects are formed in a disc around the central black hole. The distribution of compact object masses could provide information on their populations, and any IMRI detection would be exciting in demonstrating that intermediate-mass black holes exist. Spin measurements of massive black holes will enlighten our understanding of their formation history. If any electromagnetic counterparts to EMRIs are observed, it could yield an improved measurement of the Hubble constant. EMRIs could also be used to confirm whether massive objects are indeed Kerr black holes, as generally assumed, and to test strong-field general relativity. 


\section{Acknowledgments}

This work is a result of the meeting LISA Astro-GR@AEI7 organized by PAS which took place at the Max-Planck Institut für Gravitationsphysik (Albert Einstein-Institut) from 18th22nd September 2006. We thank all participants for the useful discussions during the meeting which made this article possible. The work of PAS has been supported in the framework of the Third Level Agreement between the DFG and the Instituto de Astrofísica de Canarias (IAC). JG's work was supported by St Catharine's College. The work of MF is funded through the PPARC rolling grant at the Institute of Astronomy (IoA) in Cambridge. MCM acknowledges the National Science Foundation grant AST0607428. IM would like to thank the Brinson Foundation, NASA grant NNG04GK98G and NSF grant PHY-0601459 for financial support. The work of CC was carried out at the Jet Propulsion Laboratory, California Institute of Technology and was sponsored by the National Aeronautics and Space Administration. PAS is indebted to all the members of the Astrophysical relativity group of the AEI and especially with Ute Schlichting for her help in the organization, which was crucial for the success of the meeting.

\section{References}

[1] Aarseth S J 1999 Publ. Astron. Soc. Pac. 111 1333-46

[2] Aarseth S J 2003 Gravitational N-Body Simulations: Tools and Algorithms (Cambridge, UK: Cambridge University Press) ISBN 0521432723

[3] Aarseth S J 2006 Modelling Dense Stellar Systems, 26th meeting of the IAU, Joint Discussion 14, 22-23 August 2006, Prague, Czech Republic, JD14, 1, 14

[4] Alexander T 1999 Astrophys. J. 527 835-50

[5] Alexander T 2005 Phys. Rep. 419 65-142

[6] Alexander T and Hopman C 2003 Astrophys. J. Lett. 590 L29-32

[7] Aller M C and Richstone D 2002 Astron.J. 124 3035-41

[8] Amaro-Seoane P and Spurzem R 2001 Mon. Not. R. Astron. Soc. 327 995-1003

[9] Amaro-Seoane P 2004 PhD Thesis Heidelberg University

[10] Amaro-Seoane P, Freitag M and Spurzem R 2004 Mon. Not. R. Astron. Soc. 352 655-72

[11] Amaro-Seoane P and Freitag M 2006 Astrophys. J. Lett. 653 L53-6

[12] Amaro-Seoane P, Freitag M, Rasio F A and Miller M C 2007 in preparation

[13] Amaro-Seoane P, Kupi G and Fregeau J M 2006 in preparation

[14] Armstrong J W, Estabrook F B and Tinto M 1999 Astrophys. J. 527814

[15] Arnaud K A et al 2006 Proc. 6th Int. LISA Symp. (Preprint gr-qc/0609105)

[16] Arnaud K A et al 2006 Proc. 6th Int. LISA Symp. (Preprint gr-qc/0609106)

[17] Arnaud K A et al 2007 Preprint gr-qc/0701170 v3

[18] Babak S V, Fang H, Gair J R, Glampedakis K and Hughes S A 2007 Phys. Rev. D 75024005 (Preprint gr-qc/0607007)

[19] Bahcall J N and Wolf R A 1976 Astrophys. J. 209 214-32

[20] Bahcall J N and Wolf R A 1977 Astrophys. J. $216883-907$

[21] Baker J G, Centrella J, Choi D-I, Koppitz M and van Meter J 2006 Phys. Rev. D 73104002

[22] Baker J G, Boggs W D, Centrella J, Kelly B J, McWilliams S T, Miller M C and van Meter J R 2007 Modeling kicks from the merger of non-precessing black-hole binaries Preprint astro-ph/0702390

[23] Ballero S K, Kroupa P and Matteucci F 2007 Preprint astro-ph/0702047

[24] Bandyopadhyay R M 2006 Proc. 6th Microquasar Workshop: Microquasars and Beyond. September 18-22, 2006, Como, Italy p 13.1

[25] Barack L, Creighton T, Cutler C, Gair J, Larson S, Phinney E S, Thorne K S and Vallisneri M 2003 http://www.tapir.caltech.edu/listwg1/EMRI/LISTEMRIreport.pdf

[26] Barack L and Cutler C 2004 Phys. Rev. D 69082005

[27] Barack L and Cutler C 2004 Phys. Rev. D 70122002

7 For online talks and slides please see http://www.aei.mpg.de/ pau/LISA_Astro-GR@AEI. 
[28] Barack L and Cutler C 2007 Preprint gr-qc/0612029

[29] Barack L and Lousto C O 2005 Phys. Rev. D 72104026

[30] Barack L and Sago N 2007 Preprint gr-qc/0701069

[31] Barausse E, Rezzolla L, Petroff D and Ansorg M 2007 Preprint gr-qc/0612123

[32] Bardeen J M 1970 Nature 22664

[33] Barnes J and Hut P 1986 Nature 324446

[34] Barth A J, Ho L C, Rutledge R E and Sargent W L W 2004 Astrophys. J. 607 90-102

[35] Barth A J, Greene J E and Ho L C 2005 Astrophys. J. Lett. 619 L151-4

[36] Baumgardt H, Makino J and Ebisuzaki T 2004a Astrophys. J. 613 1133-42

[37] Baumgardt H, Makino J and Ebisuzaki T 2004b Astrophys. J. 613 1143-56

[38] Bekenstein J D 1973 Astrophys. J. 183657

[39] Belczynski K, Sadowski A and Rasio F A 2004 Astrophys. J. 611 1068-79

[40] Bender P L and Hils D 1998 Bull. Am. Astron. Soc. 30844

[41] Berti E, Buonanno A and Will C M 2005 Phys. Rev. D 71084025

[42] Berti E, Cardoso V and Will C M 2006 Phys. Rev. D 73064030

[43] Binney J and Tremaine S 1987 Galactic Dynamics (Princeton, NJ: Princeton University Press)

[44] Blanchet L and Iyer B R 2003 Class. Quantum Grav. 20 755-76

[45] Brady P R, Creighton J D E and Thorne K S 1998 Phys. Rev. D 58061501

[46] Brown D A, Fang H, Gair J R, Li C, Lovelace G, Mandel I and Thorne K S 2006 Preprint gr-qc/0612060

[47] Brown W R, Geller M J, Kenyon S J and Kurtz M J 2005 Astrophys. J. Lett. 622 L33-6

[48] Brügmann B, Gonzalez J A, Hannam M, Husa S, Spehake U and Tichy W 2006 Preprint gr-qc/0610128

[49] Campanelli M, Lousto C O, Marronetti P and Zlochower Y 2006 Phys. Rev. Lett. 96111101

[50] Campanelli M, Lousto C O, Zlochower Y and Merritt D 2007 Maximum gravitational recoil Preprint gr-qc/0702133

[51] Carter B 1968 Commun. Math. Phys. 10280

[52] Chabrier G 2003 Publ. Astron. Soc. Pac. 115 763-95

[53] Chakrabarti S K 1996 Phys. Rev. D 532901

[54] Chandrasekhar S 1960 Radiative Transfer (New York: Dover) enlarged edition

[55] Chang J S and Cooper G 1970 J. Comput. Phys. 6 1-16

[56] Chernoff D F and Weinberg M D 1990 Astrophys. J. 351 121-56

[57] Chrzanowski P L 1975 Phys. Rev. D 112042

[58] Clutton-Brock M 1973 Astrophys. Space Sci. 2355

[59] Cohn H 1979 Astrophys. J. 234 1036-53

[60] Cohn H 1980 Astrophys. J. 242 765-71

[61] Cohn H 1985 IAU Symp. 113: Dynamics of Star Clusters pp 161-77

[62] Cohn H and Kulsrud R M 1978 Astrophys. J. 226 1087-108

[63] Collin S and Zahn J P 1999 IAU Symp. 194: Activity in Galaxies and Related Phenomena ed Y Terzian, E Khachikian and D Weedman vol 194, p 246

[64] Collins N A and Hughes S A 2004 Phys. Rev. D 69124022

[65] Cornish N J and Crowder J 2005 Phys. Rev. D 72043005

[66] Cornish N J and Porter E K 2006 Preprint gr-qc/0612091

[67] Cornish N J and Littenberg T B 2007 Preprint 0704.1808v2

[68] Cutler C 1998 Phys. Rev. D 57 7089-102

[69] Cutler C and Harms J 2006 Phys. Rev. D 73042001

[70] Cutler C, Kennefick D and Poisson E 1994 Phys. Rev. D 50 3816-3835

[71] Cutler C and Thorne K S 2002 Preprint gr-qc/0204090

[72] Danzman K et al 1998 LISA-laser interferometer space antenna, pre-phase: a report Report MPQ 233 Max-Planck-Institut für Quantenoptic

[73] Damour T and Deruelle N 1981 Phys. Lett. A 87 81-4

[74] Davies M B and King A 2005 Astrophys. J. Lett. 624 L25

[75] Deegan P and Nayakshin S 2006 J. Phys. Conf. Ser. 54 306-10

[76] de Freitas Pacheco J A, Filloux C and Regimbau T 2006 Phys. Rev. D 74023001

[77] Di Stefano R, Greiner J, Murray S and Garcia M 2001 Astrophys. J. Lett. 551 L37-40

[78] Dong X, Wang T, Yuan W, Shan H, Zhou H, Fan L, Dou L, Wang H, Wang J and Lu H 2007 Astrophys. J. $657700-5$

[79] Drasco S and Hughes S A 2006 Phys. Rev. D 73024027

[80] Drasco S, Flanagan E E and Hughes S A 2005 Class. Quantum Grav. 22 S801

[81] Drukier G A, Cohn H N, Lugger P M and Yong H 1999 Astrophys. J. 518 233-45 
[82] Duncan M J and Shapiro S L 1983 Astrophys. J. 268 565-81

[83] Ebisuzaki T, Makino J, Tsuru T G, Funato Y, Portegies Zwart S, Hut P, McMillan S, Matsushita S, Matsumoto H and Kawabe R 2001 Astrophys. J. Lett. 562 L19-22

[84] Edelmann H, Napiwotzki R, Heber U, Christlieb N and Reimers D 2005 Astrophys. J. Lett. 634 L181-4

[85] Eisenhauer F et al 2005 Astrophys. J. 628 246-59

[86] Enoki M, Inoue K T, Nagashima M and Sugiyama N 2004 Astrophys. J. 61519

[87] Esquej P, Saxton R D, Freyberg M J, Read A M, Altieri B, Sanchez-Portal M and Hasinger G 2007 Astron. Astrophys. 462 L49-52

[88] Farmer A J and Phinney E S 2003 Mon. Not. R. Astron. Soc. 3461197

[89] Fellhauer M, Kroupa P, Baumgardt H, Bien R, Boily C M, Spurzem R and Wassmer N 2000 New Astron. 5305

[90] Ferrarese L and Ford H 2005 Space Sci. Rev. 116 523-624

[91] Ferrarese L and Merritt D 2000 Astrophys. J. Lett. 539 L9-L12

[92] Ferrarese L et al 2006 Astrophys. J. Lett. 644 L21-4

[93] Fiestas J 2006 PhD Thesis Heidelberg University

[94] Fiestas J, Spurzem R and Kim E 2006 Mon. Not. R. Astron. Soc. 373 677-86

[95] Filippenko A V and Ho L C 2003 Astrophys. J. Lett. 588 L13-6

[96] Finn L S and Thorne K S 2000 Phys. Rev. D 62124021

[97] Frank J and Rees M J 1976 Mon. Not. R. Astron. Soc. 176 633-47

[98] Fregeau J M, Gürkan M A, Joshi K J and Rasio F A 2003 Astrophys. J. 593 772-87

[99] Fregeau J M, Gürkan M A and Rasio F A 2007 Astrophys. J. 640 L39-L42 (Preprint astro-ph/0512032)

[100] Fregeau J M, Larson S L, Miller M C, O’Shaughnessy R and Rasio F A 2006 Astrophys. J. 646 L135

[101] Freitag M 2000 PhD Thesis Université de Genève

[102] Freitag M 2001 Class. Quantum Grav. 18 4033-8

[103] Freitag M 2003a The Astrophysics of Gravitational Wave Sources ed J M Centrella vol 686 of AIP Conference Proceedings (New York: AIP) pp 109-12

[104] Freitag M 2003b Astrophys. J. Lett. 583 L21-4

[105] Freitag M and Benz W 2001 Astron. Astrophys. 375 711-38

[106] Freitag M and Benz W 2002 Astron. Astrophys. 394 345-74

[107] Freitag M and Benz W 2005 Mon. Not. R. Astron. Soc. 358 1133-58

[108] Freitag M, Amaro-Seoane P and Kalogera V 2006 Astrophys. J. 649 91-117

[109] Freitag M, Gürkan M A and Rasio F A 2006b Mon. Not. R. Astron. Soc. 368141

[110] Freitag M, Rasio F A and Baumgardt H 2006 Mon. Not. R. Astron. Soc. 368121

[111] Fryer C L and Kalogera V 2001 Astrophys. J. 554 548-60

[112] Gair J R 2007 in preparation

[113] Gair J R and Glampedakis K 2006 Phys. Rev. D 73064037

[114] Gair J R and Jones G J 2007 Class. Quantum Grav. 271145

[115] Gair J R and Wen L 2005 Class. Quantum Grav. 22 S1359

[116] Gair J R, Barack L, Creighton T, Cutler C, Larson S L, Phinney E S and Vallisneri M 2004 Class. Quantum Grav. $21 \mathrm{~S} 1595$

[117] Gair J R, Li C, Lovelace G, Mandel I and Fang H 2006 in preparation

[118] Gebhardt K et al 2001 M33: A galaxy with no supermassive black hole Astron. J. 122 2469-76

[119] Genzel R et al 2003 Astrophys. J. 594 812-32

[120] Geroch R 1970 J. Math. Phys. 112580

[121] Gezari S, Halpern J P, Komossa S, Grupe D and Leighly K M 2003 Astrophys. J. 592 42-51

[122] Ghez A M et al 2003 Astrophys. J. Lett. 586 L127-31

[123] Ghez A M, Salim S, Hornstein S D, Tanner A, Lu J R, Morris M, Becklin E E and Duchê ne G 2005 Astrophys. J. $620744-57$

[124] Giersz M 2005 Preprint astro-ph/0512606

[125] Giersz M and Heggie D C 1996 Mon. Not. R. Astron. Soc. 279 1037-56

[126] Giersz M and Spurzem R 1994 Mon. Not. R. Astron. Soc. 269241

[127] Giersz M and Spurzem R 2000 Mon. Not. R. Astron. Soc. 317581

[128] Giersz M and Spurzem R 2003 Mon. Not. R. Astron. Soc. 343 781-95

[129] Glampedakis K and Babak S 2006 Class. Quantum Grav. 234167

[130] Glampedakis K and Kennefick D 2002 Phys. Rev. D 66044002

[131] Glampedakis K, Hughes S A and Kennefick D 2002 Phys. Rev. D 66064005

[132] Goodman J 1983 Astrophys. J. 270 700-10

[133] Goodman J 2003 Mon. Not. R. Astron. Soc. 339 937-48

[134] Goodman J and Tan J C 2004 Astrophys. J. 608 108-18 
[135] Greene J E and Ho L C 2004 Astrophys. J. 610 722-36

[136] Gualandris A, Portegies Zwart S and Sipior M S 2005 Mon. Not. R. Astron. Soc. 363 223-8

[137] Gültekin K, Miller M C and Hamilton D P 2004 Astrophys. J. 616221

[138] Gültekin K, Miller M C and Hamilton D P 2006 Astrophys. J. 640156

[139] Gürkan M A, Freitag M and Rasio F A 2004 Astrophys. J. 604

[140] Gürkan M A, Fregeau J M and Rasio F A 2006 Astrophys. J. Lett. 640 L39-42

[141] Fukushige T, Makino J and Kawai A 2005 PASJ 57 1009-21

[142] Haehnelt M G 1994 Mon. Not. R. Astron. Soc. 269199

[143] Hachisu I, Nakada Y, Nomoto K and Sugimoto D 1978 Prog. Theor. Phys. 60393

[144] Halpern J P, Gezari S and Komossa S 2004 Astrophys. J. 604 572-8

[145] Hansen R O 1974 J. Math. Phys. 1546

[146] Harfst S, Gualandris A, Merritt D, Spurzem R, Portegies Zwart S and Berczik P 2006 New Astron. 12 357-77

[147] Häring N and Rix H-W 2004 Astrophys. J. Lett. 604 L89-92

[148] Heger A, Fryer C L, Woosley S E, Langer N and Hartmann D H 2003 Astrophys. J. 591 288-300

[149] Hénon M 1971a Astrophys. Space Sci. 14 151-67

[150] Hénon M 1971b Astrophys. Space Sci. 13 284-99

[151] Hénon M 1973 Dynamical Structure and Evolution of Stellar Systems, Lectures of the 3rd Advanced Course of the Swiss Society for Astronomy and Astrophysics (SSAA) ed L Martinet and M Mayor pp 183-260

[152] Hénon M 1975 IAU Symp. 69: Dynamics of Stellar Systems ed A Hayli p 133

[153] Hernquist L and Ostriker J P 1992 Astrophys. J. 386 375-97

[154] Hills J G 1975 Nature $254295-8$

[155] Hills J G 1988 Nature 331 687-9

[156] Hils D, Bender P L and Webbink R F 1990 Astrophys. J. 36075

[157] Hills J G 1991 Astron. J. 102 704-15

[158] Hils D and Bender P L 1995 Astrophys. J. Lett. 445 L7-L10

[159] Hirsch H A, Heber U, O'Toole S J and Bresolin F 2005 Astron. Astrophys. 444 L61-4

[160] Holley-Bockelmann K, Mihos J C, Sigurdsson S, Hernquist L and Norman C 2002 Astrophys. J. 567 817-27

[161] Hopman C and Alexander T 2005 Astrophys. J. 629 362-72

[162] Hopman C and Alexander T 2006a Astrophys. J. 645 1152-63

[163] Hopman C and Alexander T 2006b Astrophys. J. Lett. 645 L133-6

[164] Hopman C, Freitag M and Larson S L 2007 Mon. Not. R. Astron. Soc. 378 129-36 (Preprint astro-ph/0612337)

[165] Hughes S A 2001 Phys. Rev. D 644004

[166] Hughes S A 2000 Phys. Rev. D 61084004

[167] Hughes S A 2001 Phys. Rev. D 64064004

[168] Hughes S A 2005 Proc. 6th Int. LISA Symp. (Preprint gr-qc/0608140)

[169] Hughes S A and Blandford R D 2003 Astrophys. J. 585 L101-4

[170] Hughes S A, Drasco S, Flanagan E E and Franklin J 2005 Phys. Rev. Lett. 94221101

[171] Ivanov P B 2002 Mon. Not. R. Astron. Soc. 336 373-81

[172] Jeans J H 1915 On the theory of star-streaming and the structure of the universe Mon. Not. R. Astron. Soc. $7670-84$

[173] Johnston K V, Sigurdsson S and Hernquist L 1999 Mon. Not. R. Astron. Soc. 302 771-89

[174] Joshi K J, Rasio F A and Portegies Zwart S 2000 Astrophys. J. 540 969-82

[175] Joshi K J, Nave C P and Rasio F A 2001 Astrophys. J. 550 691-702

[176] Karas V and Šubr L 2001 Astron. Astrophys. 376 686-96

[177] Kesden M, Gair J R and Kamionkowski M 2005 Phys. Rev. D 71044015

[178] King A R, Lubow S H, Ogilvie G I and Pringle J E 2005 Mon. Not. R. Astron. Soc. 363 49-56

[179] King A R and Pringle J E 2006 Mon. Not. R. Astron. Soc. 373 L90-2

[180] King A R and Pringle J E 2007 Preprint astro-ph/0701679

[181] Komossa S 2005 Growing Black Holes: Accretion in a Cosmological Context ed A Merloni, S Nayakshin and R A Sunyaev (Berlin: Springer) pp 159-63

[182] Komossa S, Halpern J, Schartel N, Hasinger G, Santos-Lleo M and Predehl P 2004 Astrophys. J. Lett. 603 L17-20

[183] Kroupa P 2002 Science 295 82-91

[184] Kustaanheimo P E and Stiefel E L 1965 J. Reine Angew. Math. 218 204-19

[185] Kupi G, Amaro-Seoane P and Spurzem R 2006 Mon. Not. R. Astron. Soc. 371L 45K

[186] Lang R N and Hughes S A 2006 Phys. Rev. D 74122001

[187] Larson S L, Hiscock W A and Hellings R W 2000 Phys. Rev. D 62062001

[188] Lauer T R, Faber S M, Ajhar E A, Grillmair C J and Scowen P A 1998 Astron. J. 116 2263-86 
[189] Laycock S, Grindlay J, van den Berg M, Zhao P, Hong J, Koenig X, Schlegel E M and Persson S E 2005 Astrophys. J. Lett. 634 L53-6

[190] Levin Y 2003 Preprint astro-ph/0307084

[191] Levin Y 2007 Mon. Not. R. Astron. Soc. 374515

[192] Levin Y and Beloborodov A M 2003 Astrophys. J. Lett. 590 L33-6

[193] Li C 2007 in preparation

[194] Li C and Lovelace G 2007 Preprint gr-qc/ 0702146

[195] Lightman A P and Shapiro S L 1977 Astrophys. J. 211 244-62

[196] Lin D N C and Tremaine S 1980 Astrophys. J. $242789-98$

[197] Louis P D and Spurzem R 1991 Mon. Not. R. Astron. Soc. 251 408-26

[198] Lynden-Bell D and Eggleton P P 1980 Mon. Not. R. Astron. Soc. 191 483-98

[199] Lu J R, Ghez A M, Hornstein S D, Morris M, Matthews K, Thompson D J and Becklin E E 2006 J. Phys. Conf. Ser. $\mathbf{5 4} 279-87$

[200] Magorrian J et al 1998 Astron. J. 115 2285-305

[201] Magorrian J and Tremaine S 1999 Mon. Not. R. Astron. Soc. 309 447-60

[202] Mandel I in preparation

[203] Mandel I, Brown D A, Gair J R and Miller M C 2007 in preparation

[204] Manko V S and Novikov I D 1992 Class. Quantum Grav. 92477

[205] Marconi A and Hunt L K 2003 Astrophys. J. Lett. 589 L21-4

[206] Martins F, Genzel R, Eisenhauer F, Paumard T, Ott T, Gillessen S and Trippe S 2006 J. Phys. Conf. Ser. $54266-72$

[207] Matsubayashi T, Makino J and Ebisuzaki T 2007 Astrophys. J. 656 879-96 (Preprint astro-ph/0511782)

[208] Menou K, Haiman Z and Narayanan V K 2001 Astrophys. J. 558 535-42

[209] Menou K and Quataert E 2001 Astrophys. J. Lett. 562 L137-40

[210] Merritt D 1999 Elliptical galaxy dynamics Publ. Astron. Soc. Pac. 111 129-68

[211] Merritt D 2006 Rep. Prog. Phys. 69 2513-79

[212] Merritt D, Berczik P and Laun F 2007 Astron. J. 133 553-63

[213] Merritt D, Ferrarese L and Joseph C L 2001 No supermassive black hole in M33? Science 293 1116-9

[214] Merritt D, Harfst S and Bertone G 2007 Phys. Rev. D 75043517

[215] Merritt D and Poon M Y 2004 Astrophys. J. 606 788-98

[216] Merritt D and Szell A 2006 Astrophys. J. 648 890-9

[217] Mikkola S 1997 Celest. Mech. Dyn. Astron. 68 87-104

[218] Mikkola S and Aarseth S 2002 Celest. Mech. Dyn. Astron. 84 343-54

[219] Mikkola S and Merritt D 2006 Mon. Not. R. Astron. Soc. 372 219-23

[220] Miller M C 2002 Astrophys. J. 581 438-50

[221] Miller M C and Colbert E J M 2004 Int. J. Mod. Phys. D 13 1-64

[222] Miller M C and Hamilton D P 2002 Mon. Not. R. Astron. Soc. 330232

[223] Miller M C and Hamilton D P 2002 Astrophys. J. 576894

[224] Miller J M, Fabbiano G, Miller M C and Fabian A C 2003 Astrophys. J. 585 L37

[225] Miller J M, Fabian A C and Miller M C 2004 Astrophys. J. 607931

[226] Miller J M, Fabian A C and Miller M C 2004614 L117

[227] Miller M C 2005 Astrophys. J. 618 426-31

[228] Miller M C, Freitag M, Hamilton D P and Lauburg V M 2005 Astrophys. J. Lett. 631 L117-20

[229] Milosavljević M and Loeb A 2004 Astrophys. J. Lett. 604 L45-8

[230] Milosavljevic M and Merritt D 2001 Astrophys. J. 563 34-62

[231] Milosavljevic M and Merritt D 2003 Long-term evolution of massive black hole binaries Astrophys. J. $596860-78$

[232] Mino Y 2003 Phys. Rev. D 67084027

[233] Miralda-Escudé J and Gould A 2000 Astrophys. J. 545 847-53

[234] Mouri H and Taniguchi Y 2002a Astrophys. J. 566 L17

[235] Mouri H and Taniguchi Y 2002b Astrophys. J. 580844

[236] Muno M P 2006 Preprint astro-ph/0611589

[237] Muno M P, Bauer F E, Bandyopadhyay R M and Wang Q D 2006 Astrophys. J. Suppl. 165 $173-87$

[238] Muno M P et al 2006 Astrophys. J. Lett. 636 L41-4

[239] Muno M P, Lu J R, Baganoff F K, Brandt W N, Garmire G P, Ghez A M, Hornstein S D and Morris M R 2005a Astrophys. J. $633228-39$

[240] Muno M P, Pfahl E, Baganoff F K, Brandt W N, Ghez A, Lu J and Morris M R 2005b Astrophys. J. Lett. 622 L113-6 
[241] Murphy B W, Cohn H N and Durisen R H 1991 Astrophys. J. 370 60-77

[242] Narayan R 2000 Astrophys. J. 536663

[243] Nayakshin S 2006 Mon. Not. R. Astron. Soc. 372 143-50

[244] Nayakshin S and Sunyaev R 2005 Mon. Not. R. Astron. Soc. 364 L23-7

[245] Nelemans G, Yungelson L R, Portegies Zwart S F and Verbunt F 2001 Astron. Astrophys. 365491

[246] O’Leary R M, Rasio F A, Fregeau J M, Ivanova N and O’Shaughnessy R 2006 Astrophys. J. 637937

[247] Orosz J A 2003 IAU Symposium 212: A Massive Star Odyssey: From Main Sequence to Supernova ed $\mathrm{K}$ van der Hucht, A Herrero and C Esteban pp 365-71

[248] Pakull M W and Mirioni L 2002 to appear in the proceedings of the symposium 'New Visions of the x-ray Universe in the XMM-Newton and Chandra Era' Preprint astro-ph/0202488

[249] Pakull M W and Mirioni L 2003 Revista Mexicana de Astronomia y Astrofisica Conference Series ed J Arthur and W J Henney pp 197-9

[250] Papapetrou A 1951 Proc. R. Soc. A 209248

[251] Paumard T et al 2006 Astrophys. J. 643 1011-35

[252] Perets H B, Hopman C and Alexander T 2007 Astrophys. J. 656 709-20

[253] Peters P C 1964 Phys. Rev. 136 1224-32

[254] Peters P C 1964 Phys. Rev. 136 B1224

[255] Peters P C and Mathews J 1963 Phys. Rev. 131435

[256] Pfahl E 2005 Astrophys. J. 626849

[257] Poisson E 2004 Living Rev. Relativity 76 [Online article]: cited on 7/11/2006, http://www.livingreviews. org/lrr-2004-6

[258] Poon M Y and Merritt D 2002 Astrophys. J. Lett. 568 L89-92

[259] Poon M Y and Merritt D 2004 Astrophys. J. 606 774-87

[260] Portegies Zwart S F and McMillan S L W 2000 Astrophys. J. Lett. 528 L17

[261] Portegies Zwart S F, McMillan S L W, Hut P and Makino J 2001 Mon. Not. R. Astron. Soc. 321 199-226

[262] Portegies Zwart S and McMillan S L W 2002 Astrophys. J. 576899

[263] Portegies Zwart S F, Baumgardt H, Hut P, Makino J and McMillan S L W 2004 Nat 428724

[264] Portegies Zwart S, Baumgardt H, McMillan S L W, Makino J, Hut P and Ebisuzaki T 2006 Astrophys. J. 641319

[265] Pound A, Poisson E and Nickel B G 2005 Phys. Rev. D 72124001

[266] Press W H 1977 Phys. Rev. D 15965

[267] Preto M, Merritt D and Spurzem R 2004 Astrophys. J. Lett. 613 L109-12

[268] Pretorius F 2005 Phys. Rev. Lett. 95121101

[269] Rasio F A, Fregeau J M and Joshi K J 2001 The Influence of Binaries on Stellar Population Studies, Astrophysics and Space Science Library (ASSL) Vol 264 (Dordrecht: Kluwer) p 387

[270] Rauch K P 1995 Mon. Not. R. Astron. Soc. 275 628-40

[271] Rauch K P and Ingalls B 1998 Mon. Not. R. Astron. Soc. 299 1231-41

[272] Rauch K P and Tremaine S 1996 New Astronomy 1 149-70

[273] Rees M J 1988 Nature 333 523-8

[274] Rees M J and Volonteri M 2007 to appear in Proc. IAU Symp. 238, 'Black Holes: from stars to galaxies across the range of masses' Preprint astro-ph/0701512

[275] Rosenbluth M N, MacDonald W M and Judd D L 1957 Phys. Rev. 107 1-6

[276] Rubbo L J, Holley-Bockelmann K and Finn L S 2006 Astrophys. J. Lett. 649 L25-8

[277] Ruiter A J, Belczynski K and Harrison T E 2006 Astrophys. J. Lett. 640 L167-70

[278] Ryan F D 1995 Phys. Rev. D 525707

[279] Ryan F D 1997 Phys. Rev. D 556081

[280] Ryan F D 1997 Phys. Rev. D 561845

[281] Sago N, Tanaka T, Hikida W and Nakano H 2005 Prog. Theor. Phys. 114509

[282] Sago N, Tanaka T, Hikida W, Ganz K and Nakano H 2005 Prog. Theor. Phys. 115873

[283] Schoedel R et al 2007 Astron. Astrophys. at press

[284] Sesana A, Haardt F, Madau P and Volonteri M 2005 Astrophys. J. 62323

[285] Shankar F, Salucci P, Granato G L, De Zotti G and Danese L 2004 Mon. Not. R. Astron. Soc. 354 1020-30

[286] Sigurdsson S 2003 Class. Quantum Grav. 2045

[287] Sigurdsson S and Rees M J 1997 Mon. Not. R. Astron. Soc. 284 318-26

[288] Soffel M H 1989 Relativity in Astrometry, Celestial Mechanics and Geodesy, XIV (Berlin: Springer) 208 pp

[289] Spitzer L 1987 Dynamical Evolution of Globular Clusters (Princeton, NJ: Princeton University Press)

[290] Spurzem R 1992 Reviews of Modern Astronomy vol 5, pp 161-73

[291] Spurzem R and Takahashi K 1995 Mon. Not. R. Astron. Soc. 272 772-4 
[292] Stodołkiewicz J S 1982 Acta Astron. 32 63-91

[293] Stodołkiewicz J S 1986 Acta Astron. 36 19-41

[294] Stolte A, Brandner W, Grebel E K, Lenzen R and Lagrange A-M 2005 Astrophys. J. Lett. 628 L113-7

[295] Stroeer A, Gair J R and Vecchio A 2006 Proceedings of 6th LISA Symposium submitted Preprint gr-qc/0605227

[296] Strohmayer T E and Mushotzky R F 2003 Astrophys. J. 586 L61

[297] Šubr L and Karas V 1999 Astron. Astrophys. 352 452-8

[298] Syer D and Ulmer A 1999 Mon. Not. R. Astron. Soc. 306 35-42

[299] Syer D, Clarke C J and Rees M J 1991 Mon. Not. R. Astron. Soc. 250 505-12

[300] Takahashi K 1995 PASJ 47 561-73

[301] Takahashi K 1996 PASJ 48 691-700

[302] Takahashi K 1997 PASJ 49 547-60

[303] Taniguchi Y, Shioya Y, Tsuru T G and Ikeuchi S 2000 PASJ 52533

[304] Teukolsky S A 1973 Astophys. J. 185635

[305] Teukolsky S A and Press W H 1974 Astophys. J. 193443

[306] Thorne K S 1998 Black Holes and Relativistic Stars ed R M Wald (Chicago, IL: University of Chicago Press) $\mathrm{p} 41$

[307] Tinto M and Dhurandhar S 2005 Living Rev. Relativity 84 [Online article]: cited on 7/11/2006, http://relativity.livingreviews.org/Articles/lrr-2005-4/

[308] Tremaine S et al 2002 Astrophys. J. 574 740-53

[309] Ulmer A 1999 Astrophys. J. 514 180-7

[310] Umstätter R, Christensen N, Hendry M, Meyer R, Simha V, Veitch J, Vigeland S and Woan G 2005 Phys. Rev. D 72022001

[311] Vallisneri M 2005 Phys. Rev. D 71022001

[312] Valluri M, Ferrarese L, Merritt D and Joseph C L 2005 The low end of the supermassive black hole mass function: constraining the mass of a nuclear black hole in NGC 205 via stellar kinematics Astrophys. J. 628 137-52

[313] van der Marel R P 2003 in AIP Conf. Proc. 686: The Astrophysics Gravitational Wave Sources 686115

[314] Vecchio A 2004 Phys. Rev. D 70042001

[315] Volonteri M 2007 Gravitational recoil: signatures on the massive black hole population Preprint astro-ph/0703180

[316] Volonteri M, Madau P, Quataert E and Rees M J 2005 Astrophys. J. 620 69-77

[317] Wang J and Merritt D 2004 Astrophys. J. 600 149-61

[318] Wehner E H and Harris W E 2006 Astrophys. J. Lett. 644 L17-20

[319] Wen L and Gair J R 2005 Class. Quantum Grav. 22 S445

[320] Wickham E D L, Stroeer A and Vecchio A 2006 Preprint gr-qc/0605071

[321] Will C M and Yunes N 2004 Class. Quantum Grav. 214367

[322] Wyithe J S B and Loeb A 2003 Astrophys. J. 590691

[323] Yu Q and Tremaine S 2003 Astrophys. J. 599 1129-38

[324] Zhang W, Woosley S E and Heger A 2007 Preprint astro-ph/0701083

[325] Zare K 1974 Celest. Mech. 10 207-15 